\title{
Regulation of fungal polar tip extension through NDR
}

\section{kinase signalling}

\author{
Dissertation \\ zur Erlangung des Doktorgrades \\ der Mathematisch-Naturwissenschaftlichen Fakultäten \\ der Georg-August-Universität zu Göttingen
}

vorgelegt von

Sabine März

aus Weimar

Göttingen 2009 
Die vorliegende Arbeit wurde von August 2005 bis September 2009 in der Abteilung Molekulare Mikrobiologie und Genetik unter Anleitung von Prof. Dr. Gerhard H. Braus am Institut für Mikrobiologie und Genetik der Georg-August-Universität zu Göttingen angefertigt.

D7

Referent:

Prof. G. H. Braus

Korreferent:

Prof. S. Pöggeler

Tag der mündlichen Prüfung: $\quad$ 23.10.2009 
In der Wissenschaft gleichen wir alle nur den Kindern, die am Rande des Wissens hie und da einen Kiesel aufheben, während sich der weite Ozean des Unbekannten vor unseren Augen erstreckt.

Isaac Newton (1643-1727) 
Teile der Arbeit wurden bereits veröffentlicht

Maerz, S., A. Dettmann, C. Ziv, Y. Liu, O. Valerius, O. Yarden \& S. Seiler, (2009a) Two NDR kinase - MOB complexes function as distinct modules during septum formation and tip extension in Neurospora crassa. Mol Microbiol.

Maerz, S., Y. Funakoshi, Y. Negishi, T. Suzuki \& S. Seiler, (2010) The Neurospora peptide:N-glycanase ortholog PNG1 is essential for cell polarity despite its lack of enzymatic activity. J Biol Chem 285: 2326-2332.

Maerz, S., C. Ziv, N. Vogt, K. Helmstaedt, N. Cohen, R. Gorovits, O. Yarden \& S. Seiler, (2008) The nuclear Dbf2-related kinase COT1 and the mitogen-activated protein kinases MAK1 and MAK2 genetically interact to regulate filamentous growth, hyphal fusion and sexual development in Neurospora crassa. Genetics 179: 13131325.

Ziv, C., G. Kra-Oz, R. Gorovits, S. Marz, S. Seiler \& O. Yarden, (2009b) Cell elongation and branching are regulated by differential phosphorylation states of the nuclear Dbf2-related kinase COT1 in Neurospora crassa. Mol Microbiol 74: 974-989. 


\section{TABLE OF ConTENTS}

SUMMARY

ZUSAMMENFASSUNG

\section{CHAPTER I}

\section{INTRODUCTION}

1. Nuclear Dbf2 related kinases 4

1.1. Common regulators of NDR kinase activity $-\mathrm{MOB}$ proteins and germinal centre kinases 5

1.2. Phosphoregulation of NDR kinases 7

2. NDR kinase networks in unicellular eukaryotes $\quad 8$

2.1. RAM and MOR - morphogenesis networks in yeasts 8

2.2. MEN and SIN - coordination point of mitotic exit and cytokinesis in yeasts 11

3. Animal NDR kinase networks - dissolving frontiers in function, distribution and organization $\quad 13$

$\begin{array}{ll}\text { 4. The necessity and relevance of other model organism } & 17\end{array}$

$\begin{array}{lr}\text { 5. NDR kinase networks in filamentous fungi } & 18\end{array}$

$\begin{array}{lr}\text { 6. Aims of this work } & 20\end{array}$

$\begin{array}{lr}\text { References } & 21\end{array}$

\section{CHAPTER II}

THE NDR KINASE COT1, AND THE MAP KINASES MAK1 AND MAK2

GENETICALLY INTERACT TO REGULATE FILAMENTOUS GROWTH, HYPHAL FUSION AND SEXUAL DEVELOPMENT IN NEUROSPORA CRASSA

Abstract

Introduction 
Deletion of mak2 is accompanied by a reduction in PKA activity

The three $N$. crassa MAP kinases act as three distinct modules during growth and development

Discussion

Material and Methods

Strains, media and growth conditions

\section{CHAPTER III}

\section{TWO NDR KINASE - MOB COMPLEXES FUNCTION AS DISTINCT MODULES DURING SEPTUM FORMATION AND TIP EXTENSION IN NEUROSPORA CRASSA 61}

Abstract

Results

65

Three types of MOB proteins with distinct functions are present in filamentous fungi

MOB1-DBF2 and MOB2A/2B-COT1 complexes function as distinct modules during septation and tip growth

MOB2 proteins affect kinase activity and COT1 stability

MOB2 binding is required, but not sufficient for COT1 activation

Discussion 


\section{CHAPTER IV}

\section{ACTIVATION OF THE NDR KINASE COT1 INVOLVES THE GC KINASE POD6, MOB CO-ACTIVATORS AND DISTINCT PHOSPHORYLATION EVENTS

Ser417 is the major autophosphorylation site of COT1

POD6 is involved in hydrophobic motif phosphorylation of COT1

The interaction of COT1 with MOB2 does not require functional COT1 or the presence of POD6

In vitro COT1 activity does not correlate with its in vivo function

A conformational change induced through hydrophobic motif phosphorylation is required for full activation of COT1 


\section{SUMMARY}

NDR kinases play an important role in cell differentiation and morphogenesis. Until now, not much is known about the regulation of NDR kinases and the cross-communication between individual NDR kinase signalling modules. In the filamentous fungus Neurospora crassa the NDR kinase COT1 is involved in the coordination of polar hyphal tip extension. Loss of function of COT1 leads to cessation of hyphal tip extension and to a compact and hyperbranched phenotype.

Within the course of this work I showed that the MAPK (mitogen-activated protein kinases) MAK1 and MAK2 genetically interact with the COT1 pathway. mak-2 is able to suppress the cot-1 defects by reducing the activity of PKA (protein kinase A). In addition, activation of MAK1 in a cot-1 background partially suppressed the defects of the mak-2 signal transduction pathway. These genetic data indicate extensive crosstalk between the MAK1/MAK2 pathways and COT1 signalling.

In order to gain catalytic activity, NDR kinases need to form a complex with MOB proteins. The genome of $N$. crassa contains four MOB proteins (MOB1, MOB2A, MOB2B, and MOB3) and two NDR kinases COT1 and DBF2. Interaction studies demonstrate that both МOB2 proteins interact with the $\mathrm{N}$-terminus of СOT1 and regulate the activity and the protein stability of COT1. MOB1 forms a complex with DBF2, which is essential for septum formation, and plays an important role during conidiation and sexual development. Further evidence is provided for a function of MOB3 that is unrelated to those of the two identified NDR-MOB complexes in N. crassa.

In addition to the association of NDR kinases with MOB proteins, they need to be phosphorylated at two conserved sites to become fully active. Within the scope of this work S417 within the activation segment of COT1 was highlighted as a cis autophosphorylation site. The kinase POD6 is involved in the phosphorylation of T589 within the hydrophobic motif. In vitro kinase assays performed with COT1 variants precipitated from different mutant backgrounds indicate that kinase activity does not correlate with the in vivo function of COT1, which was determined by quantification of the growth rates of the respective mutant strains. These discrepancies are summarized in a multistep activation model of COT1 including conformational changes and altered localization. 


\section{ZUSAMMENFASSUNG}

NDR Kinasen spielen eine wichtige Rolle bei der Zelldifferenzierung und morphologischen Prozessen. Über die Regulation von NDR Kinasen und die Vernetzung von NDR KinaseSignaltransduktionsmodulen ist bisher wenig bekannt. In dem filamentösen Pilz Neurospora crassa ist die NDR Kinase COT1 an der Regulation des polaren Hyphenwachstums beteiligt. Ein Funktionsverlust von COT1 führt zu einem Stopp des Spitzenwachstums und zu einem kompakten, stark verzweigten Phänotyp Im Rahmen dieser Arbeit konnte gezeigt werden, dass die MAPK (Mitogen-aktivierten Proteinkinasen) MAK1 und MAK2 und deren Signalkaskaden auf genetischer Ebene mit dem COT1-Signalweg interagieren. mak-2 ist in der Lage den cot-1 Phänotyp durch eine Reduktion der PKA (Proteinkinase A) Aktivität zu supprimieren. Die Aktivierung von MAK1 in einem cot-1 Hintergrund wiederum unterdrückt partiell Defekte des mak-2 Signalweges, wie Fusions- und Wachstumsdefekte oder das Unvermögen als weiblicher Paarungspartner zu dienen. Die dargestellten Daten liefern einen Hinweis für eine Vernetzung des MAK1 bzw. MAK2 Signalweges mit der NDR Kinase COT1.

Um katalytisch aktiv sein zu können, müssen NDR Kinasen zusammen mit einem MOB Protein, von denen es in N. crassa vier gibt (MOB1, MOB2A, MOB2B, und MOB3), einen Komplex bilden. Hier konnte gezeigt werden, dass die beiden MOB2 Proteine mit dem $\mathrm{N}$ Terminus von COT1 interagieren und wichtig für die Aktivität und die Proteinstabilität von COT1 sind. MOB1 hingegen bildet einen Komplex mit DBF2, einer weiteren NDR Kinase in N. crassa, welche essentiell für die Septenbildung ist und eine wichtige Rolle bei der Konidienbildung und der sexuellen Entwicklung spielt. Für MOB3 ergab sich kein funktioneller Bezug zu den anderen drei MOB Proteinen oder den beiden NDR Kinasen.

Neben der MOB-Assoziation benötigen NDR Kinasen für ihre Aktivierung eine Phosphorylierung an zwei konservierten Aminosäureresten. Im Rahmen dieser Arbeit wurde die Aminosäure S417 im Aktivierungssegment von COT1 als cis Autophosphorylierungsstelle identifiziert. An der Phosphorylierung des zweiten konservierten Restes T589 im hydrophoben Motiv ist die Kinase POD6 beteiligt. Die Ergebnisse von in vitro Kinaseaktivitätsmessungen von COT1 und verschiedenen COT1Mutationsvarianten korrelieren nicht immer mit der durch Wachstumsraten bestimmten in vivo Funktion. Ein mehrstufiges Aktivierungsmodell von COT1, welches Änderungen der Lokalisierung und der Konformation durch Phosphorylierung einbezieht, versucht diese Diskrepanz zu erklären. 


\section{CHAPTER I}

\section{Introduction}

Establishment and maintenance of cellular polarity are important and fundamental processes in eukaryotes, which have to be coordinated with cell division, differentiation, and cell growth. Although extent of polarity can vary from a less pronounced cell shape as it can be seen in yeast cells to highly polarized cells like neurons, germinating pollen tubes or cells of filamentous fungi, the underlying molecular mechanisms seem conserved in eukaryotes. Various signal transduction pathways like PKA (protein kinase A) signalling or MAPK (mitogen activated protein kinase) cascades regulate polarized growth to allow proper development of different cells and organisms. Perturbances in these highly balanced signal cascades lead to severe morphological and developmental failures like cell separation defects in yeast, hyperbranching of fungal hyphae or tumorigenesis.

Signalling pathways frequently contain one or several protein kinases. Based on conservation of the 12 subdomains of the catalytic core, kinases are subdivided into distinct groups (Manning et al., 2002, Hanks \& Hunter, 1995). Two major groups can be distinguished based on the substrate amino acid that is phosphorylated: tyrosine and serine/ threonine specific kinases. The latter encompass several well-known groups like the Erk-/ MAP-kinase family and the STE family. Both families contain kinases of the MAP kinase signalling cascade. Another important group of serine/ threonine specific kinases is the $A G C$ (for protein kinase $\underline{A}, \underline{G}$, and $\underline{C}$ ) superfamily with members like the cAMPdependent kinase PKA, and PKC. 


\section{Nuclear Dbf2 related kinases}

One group of kinases with an important role in regulation of cellular polarity and cell division are nuclear Dbf2p related (NDR) kinases. These kinases are highly conserved from yeast to human (Table I-1) and belong to the AGC class of serine/threonine protein kinases because of their structural similarities within their kinase domains (Manning et al., 2002, Hanks \& Hunter, 1995, Millward et al., 1995).

NDR kinases exhibit a unique feature within the class of AGC kinases. Within their catalytic domain - between subdomain VII and VIII -an insert of 30-60 amino acids exists (Millward et al., 1995, Verde et al., 1998, Yarden et al., 1992, Bidlingmaier et al., 2001). This insertion is thought to possess an auto-inhibitory function that is mediated through the high content of basic (positively charged) amino acid in its C-terminal region (Bichsel et al., 2004). For human NDR1 the insert was shown to carry a nuclear localization sequence (NLS) (Millward et al., 1995). N-terminally of the kinase domain, NDR kinases contain a conserved basic region, which functions as dimerization domain and binding platform for other regulatory proteins (Millward et al., 1998, Hou et al., 2004, He et al., 2005a, Hergovich et al., 2006, Ponchon et al., 2004).

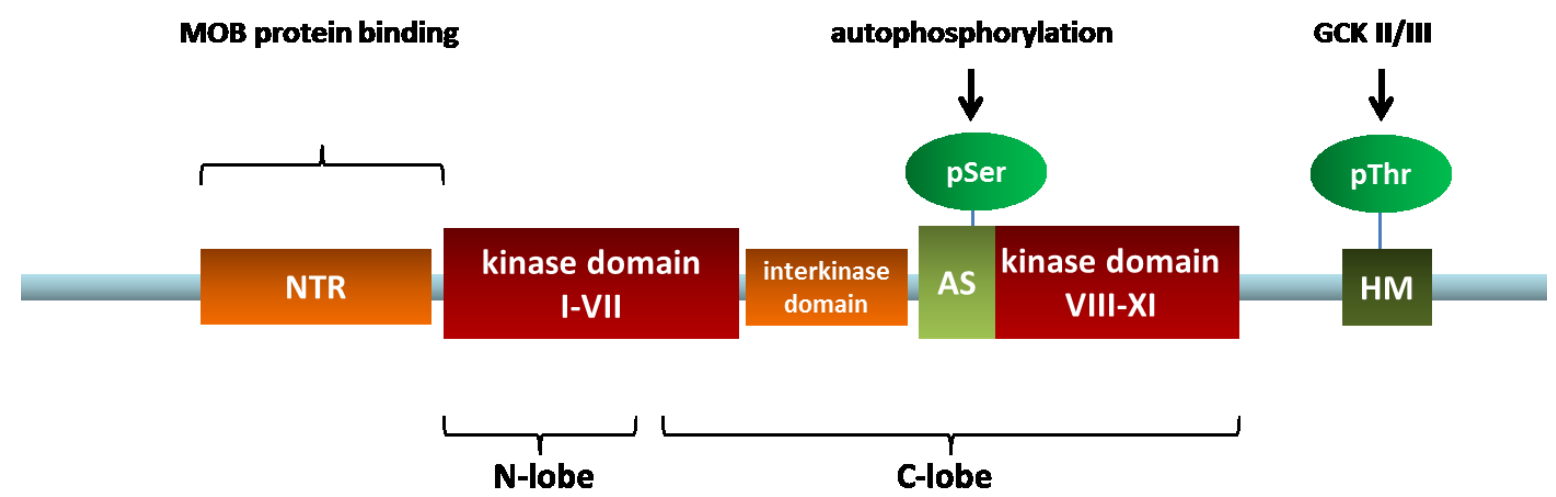

Figure I-1 General domain structure of NDR kinases. At the N-terminus NDR kinases possess a protein binding region e.g. for $\mathrm{MOB}$ proteins called N-terminal regulatory domain (NTR). The kinase domain is interrupted by the interkinase domain, an insertion of 30-60 amino acids. Subsequent to the interkinase domain the activation segment (AS) is located which harbours a conserved serine residue. This serine residue and a threonine residue within the hydrophobic motif (HM) at the C-terminus become phosphorylated upon activation of the kinase.

NDR kinases build a functional triad with $\mathrm{MOB}$ proteins and germinal centre kinases 
(GCKs), which are the core components in NDR kinase networks regulating the maintenance of cellular polarity and morphology. Prominent examples of pathways encompassing the triad of NDR, GCK, and MOB are the RAM and RAM-like networks in different yeasts, Drosophila's Hippo-pathway or the NDR pathway in mammals.

\subsection{Common regulators of NDR kinase activity -MOB proteins and germinal centre kinases}

MOB proteins are highly conserved non-catalytic proteins, which can be found in all eukaryotes. They are characterized by their mob/phocein domain. Sequence comparisons of the MOB core domain revealed that these proteins cluster in three subgroups - the MOB1-like and the MOB2-like (according to their relationship with Saccharomyces cerevisiae Mob1p or Mob2p), and the MOB3/phocein like proteins (Mrkobrada et al., 2006). Members of the phocein subfamily, the most divergent group, were described as components of several complexes consisting of striatin, protein phosphatase 2 A (PP2A) and GCKs, but they also interact with nucleoside diphosphate kinase and dynamin (Moreno et al., 2001, Baillat et al., 2001, Baillat et al., 2002, Benoist et al., 2006, Goudreault et al., 2009).

While phoceins seem not to function in concert with NDR kinases, MOB1- and MOB2-like proteins bind to and are essential for stimulating the catalytic activity of NDR kinases (Bichsel et al., 2004, Weiss et al., 2002, Hou et al., 2004). The interaction of NDR kinases with MOB proteins occurs via the basic $\mathrm{N}$-terminal regulatory domain of the NDR and an acidic surface area of the MOB protein (Figure 1-1), respectively (He et al., 2005a, Hergovich et al., 2006, Hou et al., 2004, Ponchon et al., 2004).

In addition to the interaction with MOB-proteins, NDR kinases function together with members of the GCK family (Emoto et al., 2006, Nelson et al., 2003, Walton et al., 2006, Chan et al., 2005, Stegert et al., 2005). Together with the family of p21-activated kinases (PAK), GCKs belong to the group of Ste20-related kinases. These kinases are involved in different cellular and developmental processes such as morphogenesis, cell cycle regulation, and apoptosis and are potential regulators of the MAP kinase cascades. PAKs and GCKs can be distinguished by the position of their kinase domains. Whereas PAKs posses a C-terminal kinase domain, the catalytic centre of GCKs resides at the $\mathrm{N}$-terminus (Dan et al., 2001). Within their N-terminal non-catalytic domain all PAK family member 
contain a CRIB (Cdc42/ Rac binding) domain, which is a binding motif for small GTPases.

(Figure I-2).

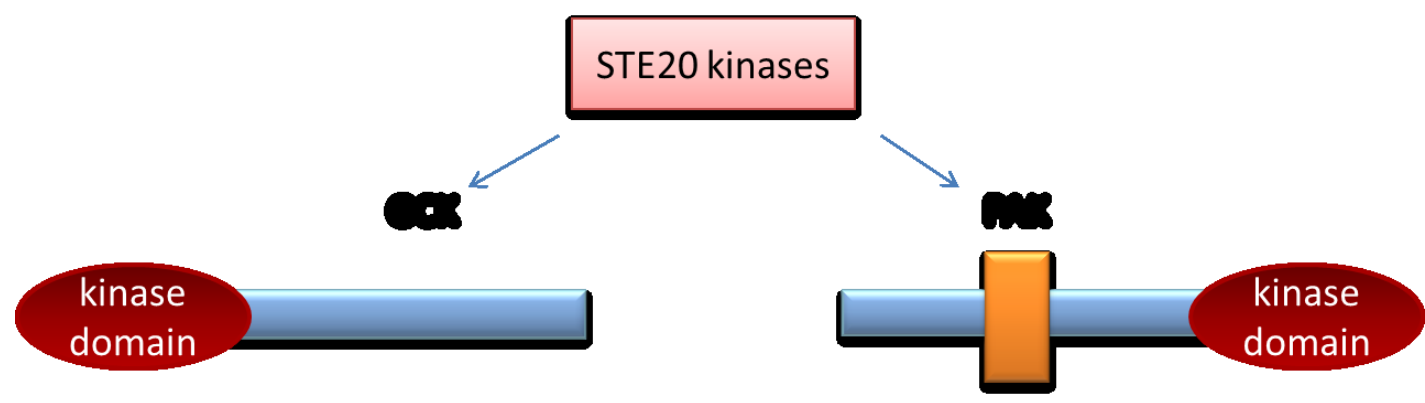

Figure I-2 The Ste20 kinases can be divided into two subfamilies GCK and PAK according to the localization of the kinase domain and by the abundance of a CRIB domain (orange).

The functionally highly diverse GCK family is subdivided into eight groups on the basis of the domain structure within the regulatory C-terminus. So far only members of the subgroups II and III, such as Drosophila's Hippo (Hpo), mammalian Mst1, Mst2, Mst3 or yeasts Kic1p, which are closely related to each other, are described to interact with and to be involved in the phosphoregulation of NDR kinases (see Table I-1).

Table I-1 NDR kinases, their functions and upstream kinases in different organisms.

\begin{tabular}{|c|c|c|c|}
\hline Organism & NDR kinase & functions & $\begin{array}{l}\text { upstream } \\
\text { kinase }\end{array}$ \\
\hline \multirow[t]{2}{*}{ S. cerevisiae } & Cbk1p & $\begin{array}{l}\text { regulation of (?) morphogenesis } \\
\text { part of the RAM network }\end{array}$ & Kic1p \\
\hline & $\begin{array}{l}\text { Dbf2p, } \\
\text { Dbf20p }\end{array}$ & $\begin{array}{l}\text { mitotic exit } \\
\text { component of MEN }\end{array}$ & Cdc15p \\
\hline \multirow[t]{2}{*}{ S. pombe } & Orb6 & $\begin{array}{l}\text { involved in the morphogenesis } \\
\text { network MOR }\end{array}$ & Nak1/ Orb3 \\
\hline & Sid2 & $\begin{array}{l}\text { initiation of septum formation } \\
\text { (SIN) }\end{array}$ & Sid1 \\
\hline \multirow[t]{2}{*}{ D. melanogaster } & Trc & $\begin{array}{l}\text { dendritic tiling, epidermal and } \\
\text { neurite outgrowth, cell shape }\end{array}$ & Hро \\
\hline & Wts & $\begin{array}{l}\text { dendritic maintenance, cell } \\
\text { cycle progression, organ size } \\
\text { control, apoptosis }\end{array}$ & Hро \\
\hline \multirow[t]{2}{*}{ H. sapiens } & NDR1/2 & $\begin{array}{l}\text { neurite outgrowth, centrosome } \\
\text { duplication }\end{array}$ & MST1/2/3 \\
\hline & LATS1/2 & $\begin{array}{l}\text { cell proliferation, centrosome } \\
\text { stability, apoptosis }\end{array}$ & MST1/2 \\
\hline \multirow[t]{2}{*}{ N. crassa } & СOT1 & maintenance of polarity & POD6 \\
\hline & Dbf2 & septation & unknown \\
\hline
\end{tabular}




\subsection{Phosphoregulation of NDR kinases}

NDR kinases are a subgroup of the AGC kinase superfamily and thus they share a similar mode of activation which involves phosphorylation at two conserved sites - the activation loop and the hydrophobic motif. The typical kinase domain encompasses 250 to 300 amino acids and consists of 12 small subdomains (Hanks \& Hunter, 1995). The $\mathrm{N}$-terminus of subdomain VIII is called the activation segment and all AGC kinases carry a conserved serine or threonine residue in this region (Figure I-1). In order to gain full catalytic activity this site needs to be autophosphorylated (for example in PKA) or - more common phosphorylated through an upstream kinase like PDK1 in the case of PKB and different PKC isoforms (Newton, 2003, Williams et al., 2000). In addition to the phosphorylation of the activation segment, most AGC kinases require a second phosphorylation event at their hydrophobic motif by autophosphorylation or an upstream kinase in order to achieve full catalytic activity (Keranen et al., 1995, Stegert et al., 2005, Yang et al., 2002). The hydrophobic motif is located C-terminally of the catalytic core and contains several hydrophobic and aromatic amino acids compassing the serine/threonine that becomes phosphorylated. In a couple AGC kinases like some atypical PKCs this phosphorylation site is replaced by either glutamic or aspartaic acid (Newton, 2003), mimicking the phosphorylated state of a serine or threonine residue. Structural analyses of AGC kinases have revealed that the (pseudo-)phosphorylated hydrophobic motif folds back and interacts with a hydrophobic pocket in the $\mathrm{N}$-terminal lobe, consisting of subdomains I-IV of the kinase domain (Biondi \& Nebreda, 2003, Frodin et al., 2002, Kannan et al., 2007, Yang et al., 2002). This interaction can take place in an intra- or intermolecular manner. Association of the phosphorylated hydrophobic motif with the hydrophobic pocket together with the phosphorylation of the activation loop results in conformational changes of the $\mathrm{N}$ - and $\mathrm{C}$-terminal lobes of the kinase. Subsequent stabilisation of the active conformation leads to a kinase with full activity.

All NDR kinases possess a serine within the activation loop and they exhibit a phosphorylatable threonine residue in the hydrophobic motif C-terminal of the kinase domain. For different members of the NDR kinase family like baker's yeast Cbk1p and human NDR1/2 it was shown that they are modulated at the serine residue in the activation segment by autophosphorylation (Jansen et al., 2006, Stegert et al., 2004, Tamaskovic et al., 2003). The threonine residue in the hydrophobic motif of several NDR 
kinases was shown to be targeted by a Ste20-like kinase which belongs to the subgroup of GCK (Chan et al., 2005, Emoto et al., 2006, Stegert et al., 2005).

\section{NDR kinase networks in unicellular eukaryotes}

Unicellular eukaryotes such as the yeasts S. cerevisiae and Schizzosaccharomyces pombe express two distinct NDR kinase networks, consisting of distinct NDR kinases, MOB proteins and upstream kinases (Table I-1). One of these signalling cascades is involved in coupling cell cycle with cell separation and is called mitotic exit network (MEN) in budding and the septation initiation network (SIN) in fission yeast, respectively (de Bettignies \& Johnston, 2003, Bardin \& Amon, 2001, Krapp et al., 2004, Krapp \& Simanis, 2005, Krapp \& Simanis, 2008, Roberts-Galbraith \& Gould, 2008). The NDR kinases Dbf2p/Dbf20p and Sid2 involved in the MEN and SIN exclusively interact with MOB1-like proteins as kinase activation factors. The second NDR kinase network regulates morphology and polar

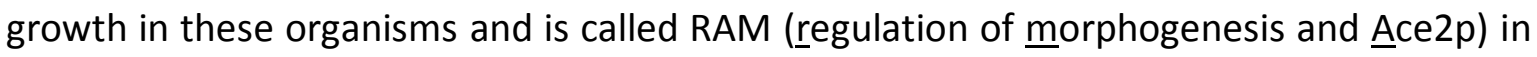
baker's yeast and MOR (ㅌorphogenesis Orb6) in fission yeast (Bogomolnaya et al., 2006, Nelson et al., 2003, Hou et al., 2003, Verde et al., 1998). The NDR kinases involved in this network, Cbk1p and ORB6, only interact with MOB2-like proteins to fulfil their function (Colman-Lerner et al., 2001, Hou et al., 2003, Weiss et al., 2002).

\subsection{RAM and MOR - morphogenesis networks in yeasts}

The RAM morphogenesis pathway in the baker's yeast consists primarily of the NDR kinase Cbk1p, the MOB protein Mob2p and the GCK Kic1p, the upstream kinase of Cbk1p. On the basis of yeast two hybrid studies and large-scale co-purification experiments the additional network components Tao3p and Hym1p, two potential scaffolding proteins, Sog $2 p$, a protein of unknown function, and the transcription factor Ace2p, a downstream effector of Cbk1p, were identified (Kurischko et al., 2005, Nelson et al., 2003, Ho et al., 2002, Ito et al., 2001). Interaction analysis revealed that the GCK Kic1p interacts with Hym $1 p$ and Sog $2 p$. The scaffold Tao3p forms a complex together with Kic1p and Cbk1p 
thereby linking the two kinases. Cbk1p bound to Mob2p is able to associate with and phosphorylate the transcription factor Ace2p (Figure I-3).

Dysfunction of either of these morphogenesis network components except Ace $2 p$ (Cbk1p, Kic1p, Mob2p, Sog2p, Hym1p, and Tao3p) leads to a loss of cellular polarity accompanied by a cell separation defect (Colman-Lerner et al., 2001, Nelson et al., 2003, Weiss et al., 2002, Racki et al., 2000) visible by the round cell morphology and clustered growth. These RAM components localize and act at the sites of cortical growth like the bud neck or mating projections, thereby influencing polar growth and morphology by yet unknown downstream targets.

Cbk1p and Mob1p do not only localize at sites of growth, but the Cbk1p-Mob2p complex can also be found in the nucleus of the daughter cell. In this compartment the kinase and its co-activator regulate the activity of the RAM effector Ace2p (Colman-Lerner et al., 2001, Nelson et al., 2003, Weiss et al., 2002). To achieve an exclusive localization in the daughter nucleus, Cbk1p needs to be phosphorylated within the hydrophobic motif at T743 by an upstream kinase, potentially through the interacting kinase Kic1p (Jansen et al., 2006).In addition phosphorylation at this site and nuclear localization of Cbk1p depends on the abundance of Ace $2 p$ (Bourens et al., 2008). Once the complex of Cbk1p and Mob2p is located in the daughter nucleus, Cbk1p phosphorylates the transcription factor Ace2p at several serine residues within its putative nuclear export sequence (NES). The phosphorylation of Ace $2 \mathrm{p}$ prevents its interaction with the nuclear export machinery (Bourens et al., 2008, Weiss et al., 2002)and therefore drives the daughter nucleus specific accumulation of Ace2p, promoting the transcriptional activity of cell wall related genes that are required for cell separation (Mazanka et al., 2008). 


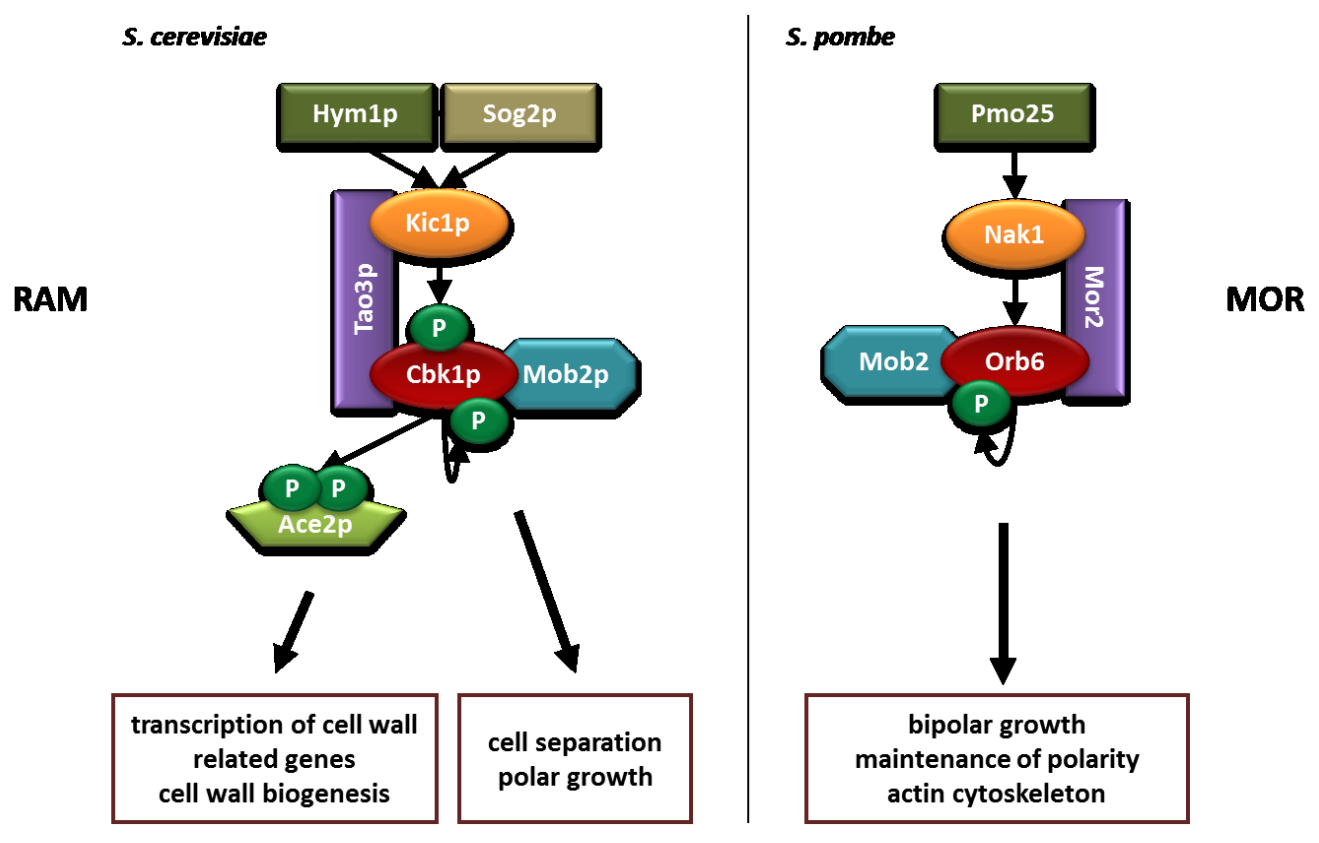

Figure I-3 The RAM and MOR networks in S. cerevisiae and S. pombe. For further details see text.

A morphogenesis network with similar central players also exists in S. pombe and is called MOR. The NDR kinase Orb6 functions together with Mob2 (Hou et al., 2003). Upstream of Orb6 the interacting GCK and Kic1p-homologue Nak1 can be found (Kanai et al., 2005, Kume et al., 2007). Both kinases Orb6 and Nak1 bind to the Tao3p homologue Mor2 (Kanai et al., 2005). A Hym1p homologue and Nak1 interacting and activating protein Pmo25 is also present (Figure 1-3), whereas a Sog2p-homologue is not described to act within the MOR network. During interphase, these proteins localize to growing cell tips and/or cell cortex and translocate to the dividing medial region during mitosis in an interdependent manner (Kanai et al., 2005, Hirata et al., 2002). Deletions or conditional mutants of either of these components are characterized by apolarly growing cells or spherically germinating spores, which lyse after a few rounds of cell division. Conditional mutants of pmo25, mor2, nak1, orb6 and mob2 are unable to re-localize F-actin to the cell ends after cell division thereby causing a disperse F-actin distribution (Hirata et al., 2002, Hou et al., 2003, Kanai et al., 2005, Verde et al., 1998), resulting in the observed polarity defect and in defective bipolar growth. This is indicating an important role of the respective proteins during bipolar growth and the establishment of polarity after cell division through the re-organization of F-actin to the sites of polar growth.

Components of the RAM/MOR networks are highly conserved and are also described in other fungi, such as Cryptococcus neoformans or the pathogen Candida albicans (Song et 
al., 2008, Walton et al., 2006, McNemar \& Fonzi, 2002). However, despite a highly conserved set of components, the morphological output can be highly different. In Cryptococcus neoformans for example, the loss of RAM components do not result in loss of polarity as observed in baker's and fission yeasts, but the respective RAM mutants form hyperpolarized cells (Walton et al., 2006).

\subsection{MEN and SIN - coordination point of mitotic exit and cytokinesis in yeasts}

Accurate completion of mitosis is coordinated by several signalling events to ensure that mitotic exit and cytokinesis do not occur before the chromosomes are segregated properly. The exit of mitosis and the onset of cytokinesis are regulated by the mitotic exit network in S. cerevisiae and the septation initiation network in S. pombe, respectively.

Central components of MEN are the small GTPase Tem1p, the protein kinase Cdc15p and the complex of the NDR kinase Dbf2p and the Mob1p, which are kept together by the scaffolding protein Nud1p (Figure I-4; Luca et al., 2001, Bardin \& Amon, 2001, Gruneberg et al., 2000). The small G-protein Tem1p binds to Nud1p, which provides a scaffolding/interaction platform for the other MEN components at the spindle pole body (SPB) (Gruneberg et al., 2000). Tem1p is kept in its inactive GDP-bound state by association with its bipartite GAP (GTPase activating protein) consisting of Bub2p and Bfa1p (Pereira et al., 2000, Geymonat et al., 2002). Once the SPB reaches the bud during mitosis, Tem1p is released from its GAP and is activated through the GEF (guanine nucleotide exchange factor) Lte1p (Pereira et al., 2000, Bardin et al., 2000). During mitosis the localization of Lte1p is restricted to the bud ensuring an asymmetric activation of the MEN components. Activation of Tem1p is followed by the recruitment of Cdc15p, Dbf2p, and Mob1p to the SPB during anaphase (Lee et al., 2001). Tem1p activates the kinase Cdc15 at the SPB (Bardin et al., 2000, Asakawa et al., 2001), which in turn is involved in phosphorylating Dbf2p and Mob1p (Mah et al., 2001). The activated Dbf2p-Mob1p complex phosphorylates the protein phosphatase Cdc14p, leading to the release of the phosphatase from the nucleus (Mohl et al., 2009). Cytoplasmic localization and subsequent activation of Cdc14p by several other kinases is necessary to inactivate mitotic CDKs (cyclin dependent kinases) and to destroy anaphase specific B-cyclins, thereby allowing the cell to exit from mitosis. During telophase Cdc15p, Dbf2p and 
Mob1p translocate to the medial ring at the cell division site, probably to promote cytokinesis (Xu et al., 2000, Yoshida \& Toh-e, 2001, Frenz et al., 2000, Luca et al., 2001). Loss of function of most MEN components leads to a late telophase arrest. In addition, a cell separation defect was observed in mob1 mutants (Luca et al., 2001).

S. cerevisiae

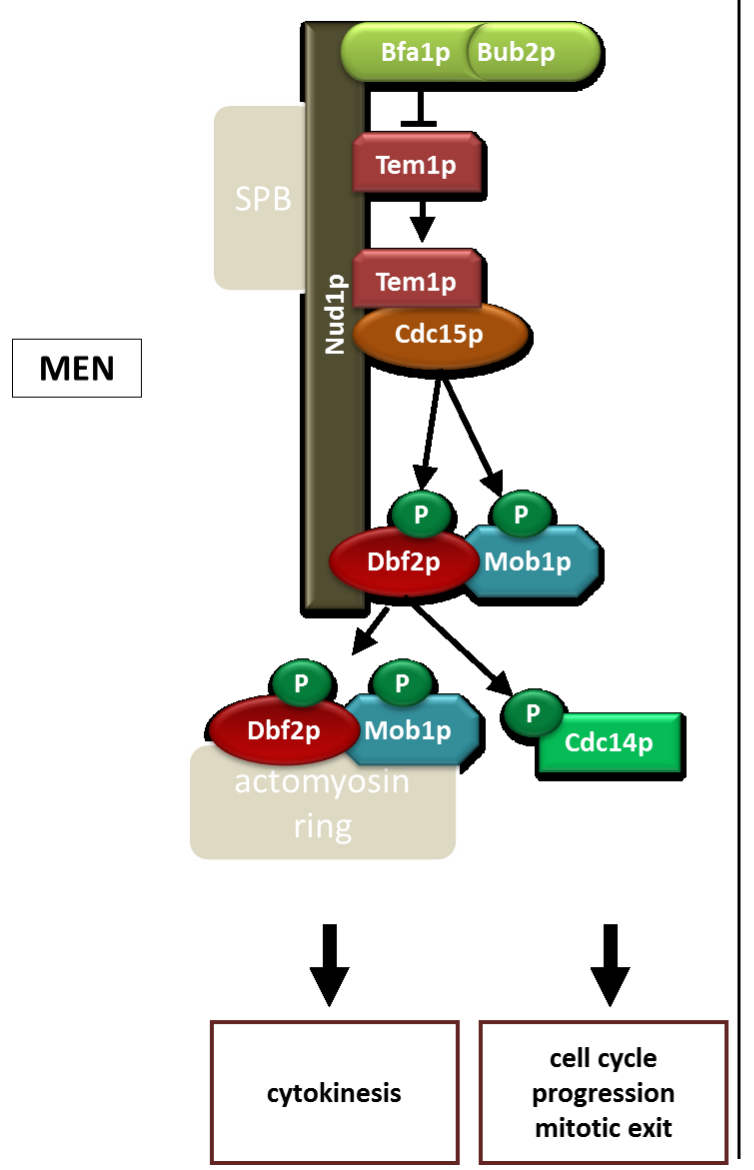

S. pombe

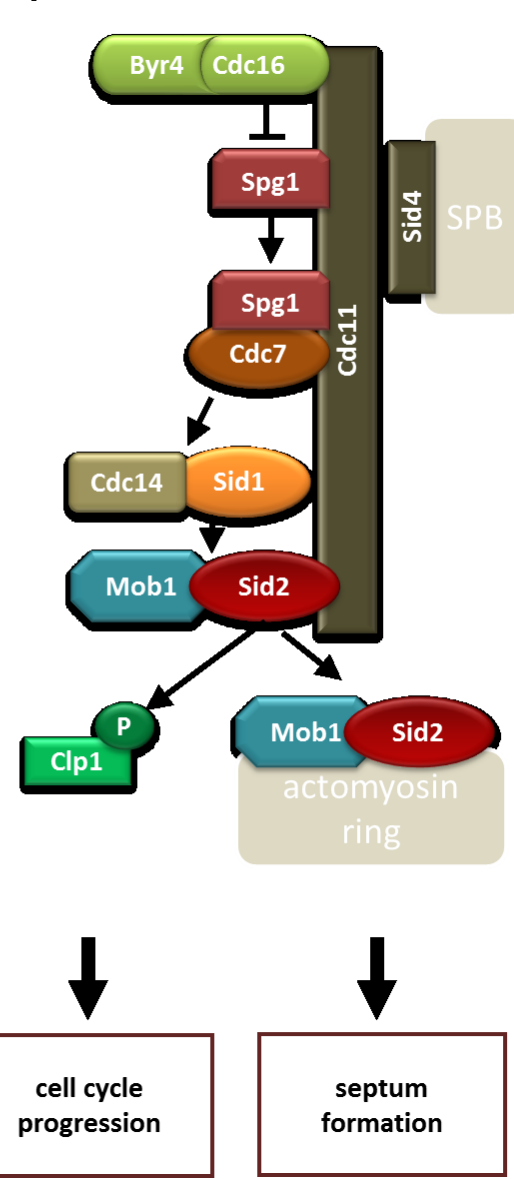

Figure I-4 The MEN of S. cerevisiae and the SIN of S. pombe are similarly arranged. For details see text.

The S. pombe SIN network is built up similarly to the MEN, nevertheless some minor differences exist (Bardin \& Amon, 2001, Krapp et al., 2004) (Figure I-4). Spg1, a small G protein that is homologues to Tem1p, localizes to the SPB through association with the scaffolding protein Cdc11 (Morrell et al., 2004). Cdc11 binds to the SPB in a complex with Cdc4. Like Tem1p, Spg1 is kept in the GDP-bound inactive state until anaphase due to binding to a dimeric GAP complex consisting of Byr4 and Cdc16 (Furge et al., 1998, Krapp et al., 2008). Upon activation of Spg1, the Cdc15p homologue Cdc7 translocates to the SPB and interacts with Spg1 (Sohrmann et al., 1998, Krapp et al., 2008, Mehta \& Gould, 2006). In addition Cdc7 promotes SPB localization of the GCK Sid1 and its associated non- 
catalytic protein Cdc14 and activates this complex during anaphase in an asymmetrical manner (Guertin et al., 2000, Guertin \& McCollum, 2001). Sid1 in turn switches on the functional complex of the NDR kinase Sid2 and its associated co-activator Mob1, which is localized at the SPB since early mitosis (Hou et al., 2004). Active Sid2 phoshorylates and regulates the protein phosphatase Clp1 in the same manner as it was shown for the $S$. cerevisae protein phosphatase Cdc14p (Chen et al., 2008). The Sid2-Mob1 complex also locates to the site of cell division promoting septum formation (Sparks et al., 1999, Salimova et al., 2000, Roberts-Galbraith \& Gould, 2008). However, unlike the S. cerevisiae MEN mutants, SIN mutants do not arrest in late mitosis. Instead conditional SIN mutants arrest prior cytokinesis after a few additional rounds of cell division due to an incomplete blockage (Roberts-Galbraith \& Gould, 2008). Therefore the mutants are elongated as well as multinucleated. The most obvious difference in the signalling cascades between MEN and SIN is that no protein homologue to the GCK Sid1 exists in baker's yeast, hence the NDR kinase Dbf2p may directly be activated by Cdc15p (Mah et al., 2001).

\section{Animal NDR kinase networks - dissolving frontiers in function, distribution and organization}

Some of the components described for SIN/MEN or RAM/MOR function are conserved and can also be found in animals. They are organized in two networks with NDR kinases as central players. One NDR pathway is primarily involved in the regulation of cell shape, while the other contributes more to cell proliferation. In contrast to yeasts, their functions partially overlap and individual components are exchangeable between these both pathways. For example, the two NDR kinase modules in Drosophila melanogaster are distinguishable by their NDR kinase and the respective scaffolding protein, but each NDR kinase is activated by the same upstream kinase and can interact with the same MOB proteins. In addition, the number of individual MOB proteins and NDR kinases is increased. The fruit fly exhibits at least three different MOB1/2-like genes and two NDR kinases, while mammals have more than five MOB1/2-like proteins and four NDR kinases. Thus, the modularity in combination with the increased number of components makes the NDR kinase networks in animals more complex and highly flexible. 
One of the two NDR kinases in D. melanogaster is called Warts (Wts) and is involved in cell proliferation (Xu et al., 1995, Watson, 1995). The Wts pathway is one of the best described NDR kinase network. Most components of the pathway from the receptor to the transcription factors and regulated genes are known (Figure I-5). Genetic studies revealed that Fat, a protocadherin, regulates the GCK Hippo (Hpo) through Expanded (Ex) and Merlin (Mer) (Bennett \& Harvey, 2006, Reddy \& Irvine, 2008, Tyler \& Baker, 2007, Willecke et al., 2006, Yin \& Pan, 2007). Ex and Mer are members of the ERM (ezrin/radixin/moesin) protein family functioning as an adaptor and signalling platform. Direct interactions between $\mathrm{Hpo}$ and the potential upstream regulators still need to be elucidated. Signalling events downstream of $\mathrm{Hpo}$ are well investigated by several biochemical experiments. The dRASSF (Drosophila Ras association family) directly associates with Hpo (Polesello et al., 2006). The interaction is mediated by the SARAH domain of dRASSF. The binding of dRASSF to Hpo negatively regulates the kinase activity of Hpo. Hpo can also interact with the scaffolding protein Salvador (Sav) via its SARAH domain. Thus Sav is competing with dRASSF for the association with Hpo (Polesello et al., 2006), which leads to the activation of Hpo. Sav also binds to Wts and thereby providing an interaction platform for the kinases Wts and Hpo. Hpo activates and phosphorylates Wts directly at T1083 within the hydrophobic motif (Emoto et al., 2006). In addition Hpo phoshorylates the MOB1-like protein Mats, thereby promoting the interaction of this coactivator with the NDR kinase Wts (Wei et al., 2007). Wts together with Mats in turn phoshorylates and thereby inactivates the transcriptional co-activator Yorkie (Yrk), so that its nuclear translocation is prevented (Oh \& Irvine, 2009, Oh \& Irvine, 2008). Nonphosphorylated Yrk activates the transcription factor Scalloped (Sd) in the nucleus (Goulev et al., 2008). Sd in turn drives the expression of proteins, which promote cell proliferation and inhibit apoptosis. Therefore the loss of Hpo, Wts, or Mats results in enhanced cell proliferation and reduced apoptosis, leading to altered organ size and tumorigenesis.

Trc the second NDR kinase in D. melanogaster is also activated and phosphorylated through Hpo in the same manner as Wts (Emoto et al., 2006) (Figure I-5). The Hpo mediated phosphorylation takes place within the hydrophobic motif at the threonine residue 449 (Emoto et al., 2006). This contrasts with the situation in yeasts, where the two different NDR kinases are phosphorylated and activated by distinct kinases. Trc does 
not bind to Sav; instead it forms a complex with another scaffold named Furry (Fry) (He et al., 2005b). Like other NDR kinases, activity of Trc depends on the binding of a MOB-like protein (He et al., 2005a). So far Trc was described to interact with the two MOB-proteins Mats (MOB1-like) and Dmob2 (MOB2-like) (He et al., 2005a). The Trc pathway regulates cell shape, polarity and morphogenesis of epidermal outgrowth like bristles and wing hairs. Dysfunction of Trc leads to splitted and deformed bristles, and multiplied hairs (Geng et al., 2000, He et al., 2005b).

Hpo as well as the NDR kinases Trc and Wts are also involved in dendrite morphogenesis. While Hpo regulates the whole process, Trc and Wts act at distinct steps during morphogenesis. Trc takes part in dendritic tiling, a process that prevents redundant tissue innervation with dendrites of identical cell types (Emoto et al., 2004, Emoto et al., 2006). Loss of function of trc leads to overlapping growth of dendrites of the same cell type and to hyperbranched dendrites. Wts instead is involved in the regulation of dendritic maintenance, and mutants of wts are characterized by a reduction of dendritic arborisation, length and amount of dendritic branches, producing a diminished dendritic field (Emoto et al., 2006). Thus both Trc and Wts act in parallel to regulate tissue innervation and dendritic morphogenesis under the control of their common upstream kinase Hpo.

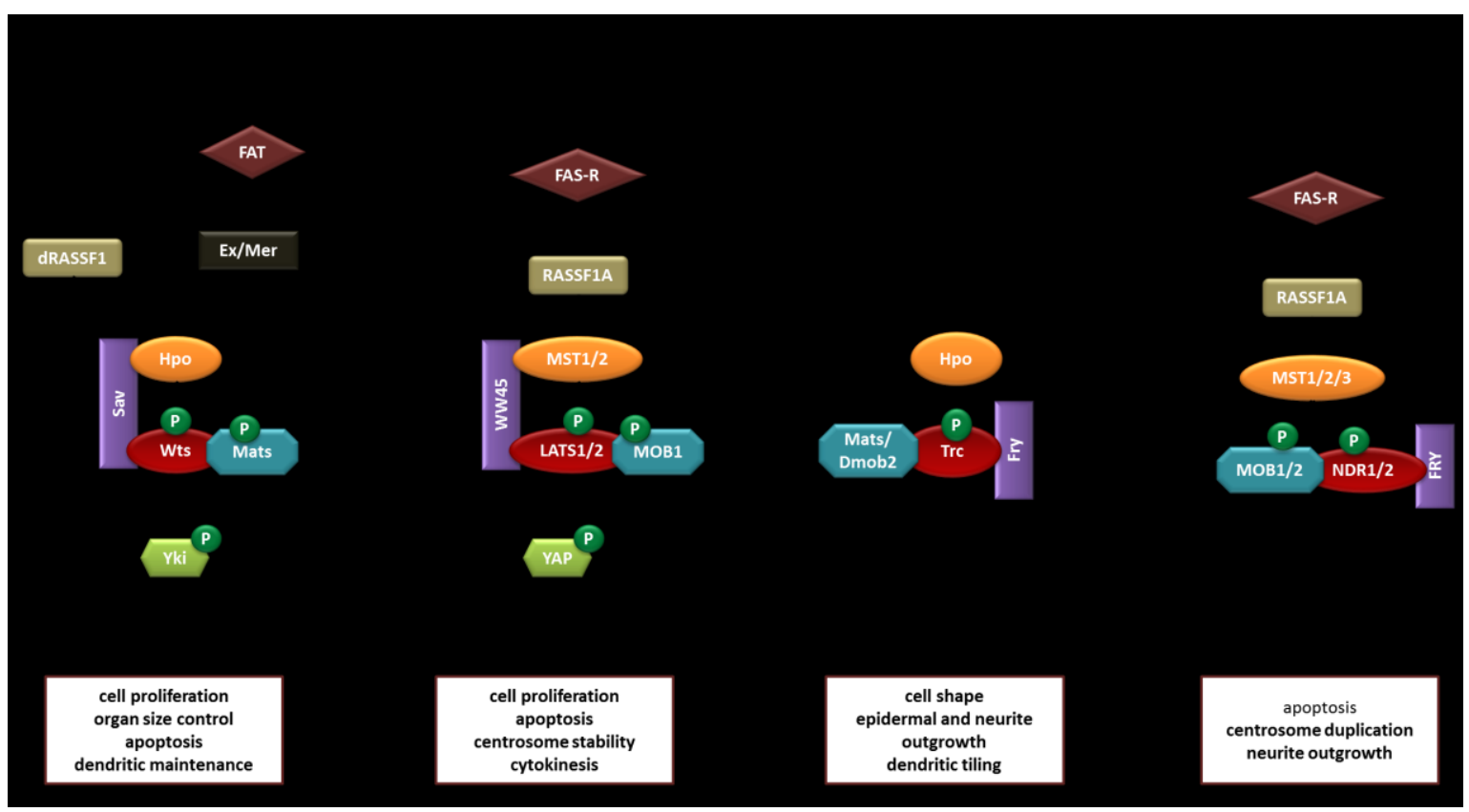

Figure I-5 Scheme of different NDR signalling pathways in D. melanogaster and mammals. See text for details. 
The mammalian NDR kinase networks are organized in a similar manner as those of the fruit fly. While the $D$. melanogaster genome contains two NDR kinases, in the human genome four NDR kinases can be found: LATS1/LATS2 (Large tumour suppressor) and NDR1/NDR2. The two kinase pairs are homologous to either Wts or Trc, respectively. In addition, at least two MOB1 and three MOB2 proteins are expressed.

The transcriptional co-activator YAP (yes associates protein), an orthologue of Yrk, becomes phosphorylated by a complex consisting of LATS1/LATS2 and MOB1, which in turn leads to a inhibition of YAP through cytoplasmic retention (Hao et al., 2008, Zhang et al., 2008, Zhao et al., 2007). While Wts is activated by one GCK, LATS1/LATS2 can be stimulated by two distinct GCKs MST1 and MST2 via hydrophobic motif phosphorylation (Chan et al., 2005). In addition MST2 is able to phosphorylate MOB1 (Hirabayashi et al., 2008). MST1/MST2 associates with a Sav-like scaffold protein called hWW45 (or hSAV) and RASSF1A (homologue to dRASSF) as described in D. melanogaster (Guo et al., 2007, Vichalkovski et al., 2008, Callus et al., 2006). Unlike dRASSF, the mammalian RASSF1A stimulates the MST1/MST2 activity in a Fas-receptor dependent manner (Vichalkovski et al., 2008). The whole LATS1/LATS2 signalling complex regulates cellular processes similar to that in the fruit fly such as cell proliferation, apoptosis, centrosome stability/maintenance, and coordination of mitotic exit with cytokinesis. Loss of LATS1/LATS2 activity leads to increased tumorigenesis, while overexpression results in a high rate of apoptotic cells.

Human NDR1 and NDR2 are the biochemically best characterized NDR kinases, but the network around these kinases is not well established. NDR1 and NDR2 play a role in neurite outgrowth and centrosome duplication (Stork et al., 2004, Hergovich et al., 2007). Recently, it was shown that NDR1/NDR2 can promote apoptosis through Fas-receptor mediated RASSF1A and subsequent MST2 activation as it was described for LATS1/LATS2 (Vichalkovski et al., 2008). Furthermore, NDR1/NDR2 can also be activated by MST1 and MST3 through hydrophobic motif phosphorylation (Chiba et al., 2009, Stegert et al., 2005, Stegert et al., 2004). Just like Trc, NDR1/NDR2 associates with both MOB1 and MOB2 type proteins and the scaffold protein FRY, a homologue of D. melanogaster Fry (Chiba et al., 2009).

In general, not much is known about substrates of NDR kinases. The yeasts transcription factor Ace2p or the transcriptional co-activators Yap and Yki of mammals and the fruit fly, 
respectively, were identified to be targeted by RAM, Wts and LATS pathways, but downstream targets of neither NDR1/NDR2 nor Trc are currently established. Thus, transcriptional regulation is one major output signal, but the regulation of other processes is likely and based on the following observations. For example, the phosphatases Cdc14p/ Clp1 become phosphorylated by the respective NDR kinases of the SIN/MEN pathways (Chen et al., 2008, Mohl et al., 2009). Several lines of evidence indicate crosstalk between NDR kinases and small GTPases in various organisms. Orb6 was described to restrict the GTPase Cdc42, a key regulator of morphology, and its guanine nucleotide exchange factor (GEF) Gef1 at the cell tips (Das et al., 2009). Budding yeast Cbk1p is involved in the regulation of Sec4p, a RabGTPase which functions in Golgi dependent glycosylation and secretion (Kurischko et al., 2008). Furthermore, Cbk1p together with Mob2p act in parallel with the Ras/ PKA signalling pathway affecting cell cycle progression and bud site selection (Schneper et al., 2004). In the fruit fly, Trc shows genetic interactions with and regulates Rac signalling in a negative manner during wing hair development and dendritic branching (He et al., 2005b, Emoto et al., 2004). In addition to the crosstalk of NDR kinases with small GTPases multiple connections with the actin cytoskeleton are provided in the fission yeast and D. melanogaster (He et al., 2005b, Hou et al., 2003, Verde et al., 1998, Geng et al., 2000). Loss of NDR function leads to altered actin organization in both organisms. A functional link of NDR kinases to MAPK cascades was established in mammals. Human NDR1 physically associates with the MAP kinase kinase kinase MEKK1, thereby inhibiting its kinase activity (Enomoto et al., 2008).

\section{The necessity and relevance of other model organism}

The findings made in $D$. melanogaster and mammals clearly indicate, that the strict functional separation of distinct NDR kinase networks observed in yeasts is not maintained in higher eukaryotes. With the ongoing complexity of the organism, the intricacy of NDR networks appears to rise. In animals some of the components are exchangeable between these two signalling modules (for cell shape or for proliferation) and the functions partially overlap. The different modules cannot be distinguished by all their components anymore, but seem discriminated only by their respective NDR kinase 
and the corresponding scaffolding protein. For example the NDR kinases Wts and LATS are closely related to Dbf2p and are involved in mitotic exit and cell division, whereas NDR kinases with higher similarity to Cbk1p (such as Trc and NDR1) regulate cell shape. This is likely due to the fact that signal transduction pathways in metazoans are more intricate and cross-linked with multiple different networks, because of their increased complexity that is visualized by different cell types, tissues and developmental stages. In contrast, the simple morphology of unicellular yeasts has allowed the identification and initial characterization of NDR kinase pathways, yet phenotypic changes are not as pronounced in yeasts as in animals. Thus, it is reasonable to dissect the NDR kinase pathways in organisms with a complexity in between unicellular yeast and animals, such as filamentous fungi. Filamentous fungi have the advantage that they are easy to handle, that they grow highly polarized and morphological changes are obvious. This makes them good model organisms to investigate the underlying mechanism of polar growth and morphogenesis.

\section{NDR kinase networks in filamentous fungi}

Until now NDR kinase modules are not well characterized in filamentous fungi. First steps in the characterization of fungal NDR kinases were made were made in Aspergillus nidulans, Neurospora crassa, and some fungal pathogens C. albicans, Ustilago maydis and Claviceps purpurea.

Nearly all components of the SIN/MEN or RAM/MOR can be found in the genome of $A$. nidulans and $N$. crassa these fungi (Table I-2). Some components of a SIN-like network were characterized in A. nidulans (Bruno et al., 2001, Harris, 2001, Harris et al., 1994, Kim et al., 2006, Kim et al., 2009), namely MOBA, a MOB1-like protein, the NDR kinase SIDB, an SID2 orthologue, the kinase SEPH, a Cdc7 homologue, the scaffolding protein SEPK, which is related to Cdc11, and the dimeric GAP forming proteins BUBA and BYR, the orthologues of Cdc16 and Byr4, respectively. All of these proteins are involved in septation and conidiation. The dimeric GAP of BUBA and BYRA is a negative regulator of these events, whereas the others positively modulate septum formation. Similar to the situation in the baker's or fission yeasts these proteins localize at the SPB. SIDB as well as 
MOBA are also found at the site of cell division, where they form a ring contracting with the developing septum, what is contrary to the observations in yeast.

Table I-2 NDR kinase network components are conserved in the filamentous fungi $\boldsymbol{A}$. nidulans and $\mathbf{N}$. crassa

\begin{tabular}{|c|c|c|c|c|}
\hline & S. cerevisiae & S. pombe & A. nidulans & N. crassa \\
\hline \multirow{6}{*}{$\begin{array}{c}\text { RAM/ } \\
\text { MOR } \\
\text { network }\end{array}$} & Hym1p & Pmo25 & HymA (ANID_03095.1) & NCU03576. \\
\hline & Kic1p & Nak1 & ANID_005674.1 & POD6 \\
\hline & Tao3p & Mor2 & ANID_00594.1 & NCU097460.3 \\
\hline & Cbk1p & Orb6 & COTA & COT1 \\
\hline & Mob2p & Mob2 & ANID_01370.1 & MOB2A (NCU03314.3) \\
\hline & & & & MOB2B (NCU07460.3) \\
\hline \multirow{9}{*}{ MEN/ SIN } & Nud1p & Cdc11 & SEPK (ANID_02459) & NCU03545.3 \\
\hline & Bfa1p & Byr4 & BYRA (ANID_09413.1) & $\begin{array}{l}\text { gene present, } \\
\text { but not annotated }\end{array}$ \\
\hline & Bub2p & Cdc16 & BUBA (ANID_07206.1) & NCU03237.3 \\
\hline & Tem1p & Spg1 & ANID_07206.1 & NCU08878.3 \\
\hline & Cdc15p & Cdc7 & SEPH (ANID_04384.1) & NCU01335.3 \\
\hline & & Cdc14 & ANID_00655.1 & NCU06636.3 \\
\hline & & Sid1 & ANID_11032.1 & NCU04096.3 \\
\hline & Mob1p & Mob1 & MOBA (ANID_06288) & MOB1 (NCU01605.3) \\
\hline & Dbf2p & SID2 & SIDB (ANID_08751.1) & NCU09071.3 \\
\hline
\end{tabular}

Only few data are available about RAM-like NDR kinase networks in filamentous fungi. COT1 of $N$. crassa, the founding member of the NDR kinase family, and COTA, the respective NDR kinase in $A$. nidulans, are both involved in the maintenance of polarity (Johns et al., 2006, Yarden et al., 1992). Loss of function of these proteins results in compact colony growth and hyperbranched hyphae due to cessation of tip extension and excessive induction of hyphal tip formation. COTA of $A$. nidulans interacts with the MOB2like protein $\mathrm{MOBB}$, and deletion of $\mathrm{MOBB}$ results in an identical phenotype as in $\triangle \cot A$ (Shi et al., 2008). COT1 was described to associate with the GCK POD6 (Seiler et al., 2006), a potential upstream kinase. Temperature sensitive mutants of both exhibit the same hyperbranched and compact phenotype at restrictive temperature.

cot-1 homologues in several additional filamentous fungi have been shown to be involved in hyphal elongation and hyperbranching, supporting the significance of this gene in the 
proper growth of filamentous fungi. Mutation or deletion of cpcot-1, a NDR gene of, the pathogen $C$. purpurea results in a hyperbranched, and compact growing phenotype as it can be observed in A. nidulans or N. crassa (Scheffer et al., 2005). In the dimorphic fungi C. albicans and U. maydis disruption of the NDR kinase genes $C B K 1$ and $u k c 1$, respectively, cause cell separation defects and a block in the transition from budding to hyphal growth (Durrenberger \& Kronstad, 1999, McNemar \& Fonzi, 2002, Song et al., 2008). However, in all the mentioned pathogens (C. albicans, C. purpurea and U. maydis), inactivation of the cot-1 homologue resulted in impaired pathogenicity.

\section{Aims of this work}

The description of cot-1 mutants (Collinge et al., 1978, Steele \& Trinci, 1977, Terenzi \& Reissig, 1967) and subsequent cloning of cot-1 (Yarden et al., 1992) has identified this NDR kinase as one of the few characterized proteins that specifically regulate polar hyphal tip extension and restriction of supernummary branch initiations. Thus, NDR kinase signalling may be a critical pathway for the understanding of the most fundamental process required for the proliferation of fungal species - filamentous growth. The available genome data revealed that nearly all components required for RAM or MOR signalling exists in N. crassa (Table I-2). So far, only the interaction of COT1 with its potential upstream kinase POD6 was described (Seiler et al., 2006), and the integration of COT1 into a cellular signalling network was missing. This work was aiming at the identification of COT1 interacting proteins and the characterization of their function for COT1, but also their impact on other signalling pathways. This work also included the analysis of key regulatory phosphorylation sites of COT1 and the establishment of a hierarchical activation model of COT1. 


\section{References}

Asakawa, K., Yoshida, S., Otake, F. \& Toh-e, A., (2001) A novel functional domain of Cdc15 kinase is required for its interaction with Tem1 GTPase in Saccharomyces cerevisiae. Genetics 157: 1437-1450.

Baillat, G., Gaillard, S., Castets, F. \& Monneron, A., (2002) Interactions of phocein with nucleoside-diphosphate kinase, Eps15, and Dynamin I. J Biol Chem 277: 1896118966.

Baillat, G., Moqrich, A., Castets, F., Baude, A., Bailly, Y., Benmerah, A. \& Monneron, A., (2001) Molecular cloning and characterization of phocein, a protein found from the Golgi complex to dendritic spines. Mol Biol Cell 12: 663-673.

Bardin, A. J. \& Amon, A., (2001) Men and sin: what's the difference? Nat Rev Mol Cell Biol 2: 815-826.

Bardin, A. J., Visintin, R. \& Amon, A., (2000) A mechanism for coupling exit from mitosis to partitioning of the nucleus. Cell 102: 21-31.

Bennett, F. C. \& Harvey, K. F., (2006) Fat cadherin modulates organ size in Drosophila via the Salvador/Warts/Hippo signaling pathway. Curr Biol 16: 2101-2110.

Benoist, M., Gaillard, S. \& Castets, F., (2006) The striatin family: a new signaling platform in dendritic spines. J Physiol Paris 99: 146-153.

Bichsel, S. J., Tamaskovic, R., Stegert, M. R. \& Hemmings, B. A., (2004) Mechanism of activation of NDR (nuclear Dbf2-related) protein kinase by the hMOB1 protein. $J$ Biol Chem 279: 35228-35235.

Bidlingmaier, S., Weiss, E. L., Seidel, C., Drubin, D. G. \& Snyder, M., (2001) The Cbk1p pathway is important for polarized cell growth and cell separation in Saccharomyces cerevisiae. Mol Cell Biol 21: 2449-2462.

Biondi, R. M. \& Nebreda, A. R., (2003) Signalling specificity of Ser/Thr protein kinases through docking-site-mediated interactions. Biochem J 372: 1-13.

Bogomolnaya, L. M., Pathak, R., Guo, J. \& Polymenis, M., (2006) Roles of the RAM signaling network in cell cycle progression in Saccharomyces cerevisiae. Curr Genet 49: 384-392.

Bourens, M., Racki, W., Becam, A. M., Panozzo, C., Boulon, S., Bertrand, E. \& Herbert, C. J., (2008) Mutations in a small region of the exportin Crm1p disrupt the daughter cell-specific nuclear localization of the transcription factor Ace $2 p$ in Saccharomyces cerevisiae. Biol Cell 100: 343-354.

Bruno, K. S., Morrell, J. L., Hamer, J. E. \& Staiger, C. J., (2001) SEPH, a Cdc7p orthologue from Aspergillus nidulans, functions upstream of actin ring formation during cytokinesis. Mol Microbiol 42: 3-12

Callus, B. A., Verhagen, A. M. \& Vaux, D. L., (2006) Association of mammalian sterile twenty kinases, Mst1 and Mst2, with hSalvador via C-terminal coiled-coil domains, leads to its stabilization and phosphorylation. FEBS J 273: 4264-4276. 
Chan, E. H., Nousiainen, M., Chalamalasetty, R. B., Schafer, A., Nigg, E. A. \& Sillje, H. H., (2005) The Ste20-like kinase Mst2 activates the human large tumor suppressor kinase Lats1. Oncogene 24: 2076-2086.

Chen, C. T., Feoktistova, A., Chen, J. S., Shim, Y. S., Clifford, D. M., Gould, K. L. \& McCollum, D., (2008) The SIN kinase Sid2 regulates cytoplasmic retention of the $S$. pombe Cdc14-like phosphatase Clp1. Curr Biol 18: 1594-1599.

Chiba, S., Ikeda, M., Katsunuma, K., Ohashi, K. \& Mizuno, K., (2009) MST2- and Furrymediated activation of NDR1 kinase is critical for precise alignment of mitotic chromosomes. Curr Biol 19: 675-681.

Collinge, A. J., Fletcher, M. H. \& Trinci, A. P. J., (1978) Physiological and cytology of septation and branching in a temperature-sensitive colonial mutant (cot-1) of Neurospora crassa. Trans. Br. Mycol. Soc. 71: 107-120.

Colman-Lerner, A., Chin, T. E. \& Brent, R., (2001) Yeast Cbk1 and Mob2 activate daughterspecific genetic programs to induce asymmetric cell fates. Cell 107: 739-750.

Dan, I., Watanabe, N. M. \& Kusumi, A., (2001) The Ste20 group kinases as regulators of MAP kinase cascades. Trends Cell Biol 11: 220-230.

Das, M., Wiley, D. J., Chen, X., Shah, K. \& Verde, F., (2009) The conserved NDR kinase Orb6 controls polarized cell growth by spatial regulation of the small GTPase Cdc42. Curr Biol 19: 1314-1319.

de Bettignies, G. \& Johnston, L. H., (2003) The mitotic exit network. Curr Biol 13: R301.

Durrenberger, F. \& Kronstad, J., (1999) The ukc1 gene encodes a protein kinase involved in morphogenesis, pathogenicity and pigment formation in Ustilago maydis. Mol Gen Genet 261: 281-289.

Emoto, K., He, Y., Ye, B., Grueber, W. B., Adler, P. N., Jan, L. Y. \& Jan, Y. N., (2004) Control of dendritic branching and tiling by the Tricornered-kinase/Furry signaling pathway in Drosophila sensory neurons. Cell 119: 245-256.

Emoto, K., Parrish, J. Z., Jan, L. Y. \& Jan, Y. N., (2006) The tumour suppressor Hippo acts with the NDR kinases in dendritic tiling and maintenance. Nature 443: 210-213.

Enomoto, A., Kido, N., Ito, M., Morita, A., Matsumoto, Y., Takamatsu, N., Hosoi, Y. \& Miyagawa, K., (2008) Negative regulation of MEKK1/2 signaling by serinethreonine kinase 38 (STK38). Oncogene 27: 1930-1938.

Frenz, L. M., Lee, S. E., Fesquet, D. \& Johnston, L. H., (2000) The budding yeast Dbf2 protein kinase localises to the centrosome and moves to the bud neck in late mitosis. J Cell Sci 113 Pt 19: 3399-3408.

Frodin, M., Antal, T. L., Dummler, B. A., Jensen, C. J., Deak, M., Gammeltoft, S. \& Biondi, R. M., (2002) A phosphoserine/threonine-binding pocket in AGC kinases and PDK1 mediates activation by hydrophobic motif phosphorylation. EMBO J 21: 53965407.

Furge, K. A., Wong, K., Armstrong, J., Balasubramanian, M. \& Albright, C. F., (1998) Byr4 and Cdc16 form a two-component GTPase-activating protein for the Spg1 GTPase that controls septation in fission yeast. Curr Biol 8: 947-954. 
Geng, W., He, B., Wang, M. \& Adler, P. N., (2000) The tricornered gene, which is required for the integrity of epidermal cell extensions, encodes the Drosophila nuclear DBF2-related kinase. Genetics 156: 1817-1828.

Geymonat, M., Spanos, A., Smith, S. J., Wheatley, E., Rittinger, K., Johnston, L. H. \& Sedgwick, S. G., (2002) Control of mitotic exit in budding yeast. In vitro regulation of Tem1 GTPase by Bub2 and Bfa1. J Biol Chem 277: 28439-28445.

Goudreault, M., D'Ambrosio, L. M., Kean, M. J., Mullin, M. J., Larsen, B. G., Sanchez, A., Chaudhry, S., Chen, G. I., Sicheri, F., Nesvizhskii, A. I., Aebersold, R., Raught, B. \& Gingras, A. C., (2009) A PP2A phosphatase high density interaction network identifies a novel striatin-interacting phosphatase and kinase complex linked to the cerebral cavernous malformation 3 (CCM3) protein. Mol Cell Proteomics 8: 157-171.

Goulev, Y., Fauny, J. D., Gonzalez-Marti, B., Flagiello, D., Silber, J. \& Zider, A., (2008) SCALLOPED interacts with YORKIE, the nuclear effector of the hippo tumorsuppressor pathway in Drosophila. Curr Biol 18: 435-441.

Gruneberg, U., Campbell, K., Simpson, C., Grindlay, J. \& Schiebel, E., (2000) Nud1p links astral microtubule organization and the control of exit from mitosis. $E M B O \mathrm{~J} 19$ : 6475-6488.

Guertin, D. A., Chang, L., Irshad, F., Gould, K. L. \& McCollum, D., (2000) The role of the sid1p kinase and cdc14p in regulating the onset of cytokinesis in fission yeast. EMBO J 19: 1803-1815.

Guertin, D. A. \& McCollum, D., (2001) Interaction between the noncatalytic region of Sid1p kinase and Cdc14p is required for full catalytic activity and localization of Sid1p. J Biol Chem 276: 28185-28189.

Guo, C., Tommasi, S., Liu, L., Yee, J. K., Dammann, R. \& Pfeifer, G. P., (2007) RASSF1A is part of a complex similar to the Drosophila Hippo/Salvador/Lats tumor-suppressor network. Curr Biol 17: 700-705.

Hanks, S. K. \& Hunter, T., (1995) Protein kinases 6. The eukaryotic protein kinase superfamily: kinase (catalytic) domain structure and classification. FASEB J 9: 576596.

Hao, Y., Chun, A., Cheung, K., Rashidi, B. \& Yang, X., (2008) Tumor suppressor LATS1 is a negative regulator of oncogene YAP. J Biol Chem 283: 5496-5509.

Harris, S. D., (2001) Septum formation in Aspergillus nidulans. Curr Opin Microbiol 4: 736739.

Harris, S. D., Morrell, J. L. \& Hamer, J. E., (1994) Identification and characterization of Aspergillus nidulans mutants defective in cytokinesis. Genetics 136: 517-532.

He, Y., Emoto, K., Fang, X., Ren, N., Tian, X., Jan, Y. N. \& Adler, P. N., (2005a) Drosophila Mob family proteins interact with the related tricornered (Trc) and warts (Wts) kinases. Mol Biol Cell 16: 4139-4152.

He, Y., Fang, X., Emoto, K., Jan, Y. N. \& Adler, P. N., (2005b) The tricornered Ser/Thr protein kinase is regulated by phosphorylation and interacts with furry during Drosophila wing hair development. Mol Biol Cell 16: 689-700. 
Hergovich, A., Lamla, S., Nigg, E. A. \& Hemmings, B. A., (2007) Centrosome-associated NDR kinase regulates centrosome duplication. Mol Cell 25: 625-634.

Hergovich, A., Schmitz, D. \& Hemmings, B. A., (2006) The human tumour suppressor LATS1 is activated by human MOB1 at the membrane. Biochem Biophys Res Commun 345: 50-58.

Hirabayashi, S., Nakagawa, K., Sumita, K., Hidaka, S., Kawai, T., Ikeda, M., Kawata, A., Ohno, K. \& Hata, Y., (2008) Threonine 74 of MOB1 is a putative key phosphorylation site by MST2 to form the scaffold to activate nuclear Dbf2-related kinase 1. Oncogene 27: 4281-4292.

Hirata, D., Kishimoto, N., Suda, M., Sogabe, Y., Nakagawa, S., Yoshida, Y., Sakai, K., Mizunuma, M., Miyakawa, T., Ishiguro, J. \& Toda, T., (2002) Fission yeast Mor2/Cps12, a protein similar to Drosophila Furry, is essential for cell morphogenesis and its mutation induces Wee1-dependent $\mathrm{G}(2)$ delay. EMBO J 21: 4863-4874.

Ho, Y., Gruhler, A., Heilbut, A., Bader, G. D., Moore, L., Adams, S. L., Millar, A., Taylor, P., Bennett, K., Boutilier, K., Yang, L., Wolting, C., Donaldson, I., Schandorff, S., Shewnarane, J., Vo, M., Taggart, J., Goudreault, M., Muskat, B., Alfarano, C., Dewar, D., Lin, Z., Michalickova, K., Willems, A. R., Sassi, H., Nielsen, P. A., Rasmussen, K. J., Andersen, J. R., Johansen, L. E., Hansen, L. H., Jespersen, H., Podtelejnikov, A., Nielsen, E., Crawford, J., Poulsen, V., Sorensen, B. D., Matthiesen, J., Hendrickson, R. C., Gleeson, F., Pawson, T., Moran, M. F., Durocher, D., Mann, M., Hogue, C. W., Figeys, D. \& Tyers, M., (2002) Systematic identification of protein complexes in Saccharomyces cerevisiae by mass spectrometry. Nature 415: 180-183.

Hou, M. C., Guertin, D. A. \& McCollum, D., (2004) Initiation of cytokinesis is controlled through multiple modes of regulation of the Sid2p-Mob1p kinase complex. Mol Cell Biol 24: 3262-3276.

Hou, M. C., Wiley, D. J., Verde, F. \& McCollum, D., (2003) Mob2p interacts with the protein kinase Orb6p to promote coordination of cell polarity with cell cycle progression. J Cell Sci 116: 125-135.

Ito, T., Chiba, T., Ozawa, R., Yoshida, M., Hattori, M. \& Sakaki, Y., (2001) A comprehensive two-hybrid analysis to explore the yeast protein interactome. Proc Natl Acad Sci U S A 98: 4569-4574.

Jansen, J. M., Barry, M. F., Yoo, C. K. \& Weiss, E. L., (2006) Phosphoregulation of Cbk1 is critical for RAM network control of transcription and morphogenesis. J Cell Biol 175: 755-766.

Johns, S. A., Leeder, A. C., Safaie, M. \& Turner, G., (2006) Depletion of Aspergillus nidulans cotA causes a severe polarity defect which is not suppressed by the nuclear migration mutation nudA2. Mol Genet Genomics 275: 593-604.

Kanai, M., Kume, K., Miyahara, K., Sakai, K., Nakamura, K., Leonhard, K., Wiley, D. J., Verde, F., Toda, T. \& Hirata, D., (2005) Fission yeast MO25 protein is localized at SPB and septum and is essential for cell morphogenesis. EMBO J 24: 3012-3025. 
Kannan, N., Haste, N., Taylor, S. S. \& Neuwald, A. F., (2007) The hallmark of AGC kinase functional divergence is its C-terminal tail, a cis-acting regulatory module. Proc Natl Acad Sci U S A 104: 1272-1277.

Keranen, L. M., Dutil, E. M. \& Newton, A. C., (1995) Protein kinase C is regulated in vivo by three functionally distinct phosphorylations. Curr Biol 5: 1394-1403.

Kim, J. M., Lu, L., Shao, R., Chin, J. \& Liu, B., (2006) Isolation of mutations that bypass the requirement of the septation initiation network for septum formation and conidiation in Aspergillus nidulans. Genetics 173: 685-696.

Kim, J. M., Zeng, C. J., Nayak, T., Shao, R., Huang, A. C., Oakley, B. R. \& Liu, B., (2009) Timely septation requires SNAD-dependent spindle pole body localization of the septation initiation network components in the filamentous fungus Aspergillus nidulans. Mol Biol Cell 20: 2874-2884.

Krapp, A., Collin, P., Cano Del Rosario, E. \& Simanis, V., (2008) Homoeostasis between the GTPase Spg1p and its GAP in the regulation of cytokinesis in S. pombe. J Cell Sci 121: 601-608.

Krapp, A., Gulli, M. P. \& Simanis, V., (2004) SIN and the art of splitting the fission yeast cell. Curr Biol 14: R722-730.

Krapp, A. \& Simanis, V., (2005) Cell division: SIN, cytokinesis and ethanol dependency. Curr Biol 15: R605-607.

Krapp, A. \& Simanis, V., (2008) An overview of the fission yeast septation initiation network (SIN). Biochem Soc Trans 36: 411-415.

Kume, K., Goshima, T., Miyahara, K., Toda, T. \& Hirata, D., (2007) A method for Pmo25associated kinase assay in fission yeast: the activity is dependent on two $\mathrm{gC}$ kinases Nak1 and Sid1. Biosci Biotechnol Biochem 71: 615-617.

Kurischko, C., Kuravi, V. K., Wannissorn, N., Nazarov, P. A., Husain, M., Zhang, C., Shokat, K. M., McCaffery, J. M. \& Luca, F. C., (2008) The yeast LATS/Ndr kinase Cbk1 regulates growth via Golgi-dependent glycosylation and secretion. Mol Biol Cell 19: 5559-5578.

Kurischko, C., Weiss, G., Ottey, M. \& Luca, F. C., (2005) A role for the Saccharomyces cerevisiae regulation of Ace 2 and polarized morphogenesis signaling network in cell integrity. Genetics 171: 443-455.

Lee, S. E., Frenz, L. M., Wells, N. J., Johnson, A. L. \& Johnston, L. H., (2001) Order of function of the budding-yeast mitotic exit-network proteins Tem1, Cdc15, Mob1, Dbf2, and Cdc5. Curr Biol 11: 784-788.

Luca, F. C., Mody, M., Kurischko, C., Roof, D. M., Giddings, T. H. \& Winey, M., (2001) Saccharomyces cerevisiae Mob1p is required for cytokinesis and mitotic exit. Mol Cell Biol 21: 6972-6983.

Mah, A. S., Jang, J. \& Deshaies, R. J., (2001) Protein kinase Cdc15 activates the Dbf2-Mob1 kinase complex. Proc Natl Acad Sci U S A 98: 7325-7330.

Manning, G., Whyte, D. B., Martinez, R., Hunter, T. \& Sudarsanam, S., (2002) The protein kinase complement of the human genome. Science 298: 1912-1934. 
Mazanka, E., Alexander, J., Yeh, B. J., Charoenpong, P., Lowery, D. M., Yaffe, M. \& Weiss, E. L., (2008) The NDR/LATS family kinase Cbk1 directly controls transcriptional asymmetry. PLoS Biol 6: e203.

McNemar, M. D. \& Fonzi, W. A., (2002) Conserved serine/threonine kinase encoded by CBK1 regulates expression of several hypha-associated transcripts and genes encoding cell wall proteins in Candida albicans. J Bacteriol 184: 2058-2061.

Mehta, S. \& Gould, K. L., (2006) Identification of functional domains within the septation initiation network kinase, Cdc7. J Biol Chem 281: 9935-9941.

Millward, T., Cron, P. \& Hemmings, B. A., (1995) Molecular cloning and characterization of a conserved nuclear serine(threonine) protein kinase. Proc Natl Acad Sci U S A 92: 5022-5026.

Millward, T. A., Heizmann, C. W., Schafer, B. W. \& Hemmings, B. A., (1998) Calcium regulation of $\mathrm{Ndr}$ protein kinase mediated by $\mathrm{S} 100$ calcium-binding proteins. EMBO J 17: 5913-5922.

Mohl, D. A., Huddleston, M. J., Collingwood, T. S., Annan, R. S. \& Deshaies, R. J., (2009) Dbf2-Mob1 drives relocalization of protein phosphatase Cdc14 to the cytoplasm during exit from mitosis. $J$ Cell Biol.

Moreno, C. S., Lane, W. S. \& Pallas, D. C., (2001) A mammalian homolog of yeast MOB1 is both a member and a putative substrate of striatin family-protein phosphatase $2 \mathrm{~A}$ complexes. J Biol Chem 276: 24253-24260.

Morrell, J. L., Tomlin, G. C., Rajagopalan, S., Venkatram, S., Feoktistova, A. S., Tasto, J. J., Mehta, S., Jennings, J. L., Link, A., Balasubramanian, M. K. \& Gould, K. L., (2004) Sid4p-Cdc11p assembles the septation initiation network and its regulators at the S. pombe SPB. Curr Biol 14: 579-584.

Mrkobrada, S., Boucher, L., Ceccarelli, D. F., Tyers, M. \& Sicheri, F., (2006) Structural and functional analysis of Saccharomyces cerevisiae Mob1. J Mol Biol 362: 430-440.

Nelson, B., Kurischko, C., Horecka, J., Mody, M., Nair, P., Pratt, L., Zougman, A., McBroom, L. D., Hughes, T. R., Boone, C. \& Luca, F. C., (2003) RAM: a conserved signaling network that regulates Ace2p transcriptional activity and polarized morphogenesis. Mol Biol Cell 14: 3782-3803.

Newton, A. C., (2003) Regulation of the ABC kinases by phosphorylation: protein kinase $C$ as a paradigm. Biochem J 370: 361-371.

Oh, H. \& Irvine, K. D., (2008) In vivo regulation of Yorkie phosphorylation and Iocalization. Development 135: 1081-1088.

Oh, H. \& Irvine, K. D., (2009) In vivo analysis of Yorkie phosphorylation sites. Oncogene 28: 1916-1927.

Pereira, G., Hofken, T., Grindlay, J., Manson, C. \& Schiebel, E., (2000) The Bub2p spindle checkpoint links nuclear migration with mitotic exit. Mol Cell 6: 1-10.

Polesello, C., Huelsmann, S., Brown, N. H. \& Tapon, N., (2006) The Drosophila RASSF homolog antagonizes the hippo pathway. Curr Biol 16: 2459-2465. 
Ponchon, L., Dumas, C., Kajava, A. V., Fesquet, D. \& Padilla, A., (2004) NMR solution structure of Mob1, a mitotic exit network protein and its interaction with an NDR kinase peptide. J Mol Biol 337: 167-182.

Racki, W. J., Becam, A. M., Nasr, F. \& Herbert, C. J., (2000) Cbk1p, a protein similar to the human myotonic dystrophy kinase, is essential for normal morphogenesis in Saccharomyces cerevisiae. EMBO J 19: 4524-4532.

Reddy, B. V. \& Irvine, K. D., (2008) The Fat and Warts signaling pathways: new insights into their regulation, mechanism and conservation. Development 135: 2827-2838.

Roberts-Galbraith, R. H. \& Gould, K. L., (2008) Stepping into the ring: the SIN takes on contractile ring assembly. Genes Dev 22: 3082-3088.

Salimova, E., Sohrmann, M., Fournier, N. \& Simanis, V., (2000) The S. pombe orthologue of the $S$. cerevisiae mob1 gene is essential and functions in signalling the onset of septum formation. J Cell Sci 113 ( Pt 10): 1695-1704.

Scheffer, J., Ziv, C., Yarden, O. \& Tudzynski, P., (2005) The COT1 homologue CPCOT1 regulates polar growth and branching and is essential for pathogenicity in Claviceps purpurea. Fungal Genet Biol 42: 107-118.

Schneper, L., Krauss, A., Miyamoto, R., Fang, S. \& Broach, J. R., (2004) The Ras/protein kinase A pathway acts in parallel with the Mob2/Cbk1 pathway to effect cell cycle progression and proper bud site selection. Eukaryot Cell 3: 108-120.

Seiler, S., Vogt, N., Ziv, C., Gorovits, R. \& Yarden, O., (2006) The STE20/germinal center kinase POD6 interacts with the NDR kinase COT1 and is involved in polar tip extension in Neurospora crassa. Mol Biol Cell 17: 4080-4092.

Shi, J., Chen, W., Liu, Q., Chen, S., Hu, H., Turner, G. \& Lu, L., (2008) Depletion of the MobB and CotA complex in Aspergillus nidulans causes defects in polarity maintenance that can be suppressed by the environment stress. Fungal Genet Biol 45: 1570-1581.

Sohrmann, M., Schmidt, S., Hagan, I. \& Simanis, V., (1998) Asymmetric segregation on spindle poles of the Schizosaccharomyces pombe septum-inducing protein kinase Cdc7p. Genes Dev 12: 84-94.

Song, Y., Cheon, S. A., Lee, K. E., Lee, S. Y., Lee, B. K., Oh, D. B., Kang, H. A. \& Kim, J. Y., (2008) Role of the RAM network in cell polarity and hyphal morphogenesis in Candida albicans. Mol Biol Cell 19: 5456-5477.

Sparks, C. A., Morphew, M. \& McCollum, D., (1999) Sid2p, a spindle pole body kinase that regulates the onset of cytokinesis. J Cell Biol 146: 777-790.

Steele, G. C. \& Trinci, A. P., (1977) Effect of temperature and temperature shifts on growth and branching of a wild type and a temperature sensitive colonial mutant (Cot 1) of Neurospora crassa. Arch Microbiol 113: 43-48.

Stegert, M. R., Hergovich, A., Tamaskovic, R., Bichsel, S. J. \& Hemmings, B. A., (2005) Regulation of NDR protein kinase by hydrophobic motif phosphorylation mediated by the mammalian Ste20-like kinase MST3. Mol Cell Biol 25: 11019-11029.

Stegert, M. R., Tamaskovic, R., Bichsel, S. J., Hergovich, A. \& Hemmings, B. A., (2004) Regulation of NDR2 protein kinase by multi-site phosphorylation and the S100B calcium-binding protein. J Biol Chem 279: 23806-23812. 
Stork, O., Zhdanov, A., Kudersky, A., Yoshikawa, T., Obata, K. \& Pape, H. C., (2004) Neuronal functions of the novel serine/threonine kinase Ndr2. J Biol Chem 279: 45773-45781.

Tamaskovic, R., Bichsel, S. J., Rogniaux, H., Stegert, M. R. \& Hemmings, B. A., (2003) Mechanism of $\mathrm{Ca2+-mediated} \mathrm{regulation} \mathrm{of} \mathrm{NDR} \mathrm{protein} \mathrm{kinase} \mathrm{through}$ autophosphorylation and phosphorylation by an upstream kinase. J Biol Chem 278: 6710-6718.

Terenzi, H. F. \& Reissig, J. L., (1967) Modifiers of the cot gene in Neurospora: the gulliver mutants. Genetics 56: 321-329.

Tyler, D. M. \& Baker, N. E., (2007) Expanded and fat regulate growth and differentiation in the Drosophila eye through multiple signaling pathways. Dev Biol 305: 187-201.

Verde, F., Wiley, D. J. \& Nurse, P., (1998) Fission yeast orb6, a ser/thr protein kinase related to mammalian rho kinase and myotonic dystrophy kinase, is required for maintenance of cell polarity and coordinates cell morphogenesis with the cell cycle. Proc Natl Acad Sci U S A 95: 7526-7531.

Vichalkovski, A., Gresko, E., Cornils, H., Hergovich, A., Schmitz, D. \& Hemmings, B. A., (2008) NDR kinase is activated by RASSF1A/MST1 in response to Fas receptor stimulation and promotes apoptosis. Curr Biol 18: 1889-1895.

Walton, F. J., Heitman, J. \& Idnurm, A., (2006) Conserved elements of the RAM signaling pathway establish cell polarity in the basidiomycete Cryptococcus neoformans in a divergent fashion from other fungi. Mol Biol Cell 17: 3768-3780.

Watson, K. L., (1995) Drosophila warts--tumor suppressor and member of the myotonic dystrophy protein kinase family. Bioessays 17: 673-676.

Wei, X., Shimizu, T. \& Lai, Z. C., (2007) Mob as tumor suppressor is activated by Hippo kinase for growth inhibition in Drosophila. EMBO J 26: 1772-1781.

Weiss, E. L., Kurischko, C., Zhang, C., Shokat, K., Drubin, D. G. \& Luca, F. C., (2002) The Saccharomyces cerevisiae Mob2p-Cbk1p kinase complex promotes polarized growth and acts with the mitotic exit network to facilitate daughter cell-specific localization of Ace2p transcription factor. J Cell Biol 158: 885-900.

Willecke, M., Hamaratoglu, F., Kango-Singh, M., Udan, R., Chen, C. L., Tao, C., Zhang, X. \& Halder, G., (2006) The fat cadherin acts through the hippo tumor-suppressor pathway to regulate tissue size. Curr Biol 16: 2090-2100.

Williams, M. R., Arthur, J. S., Balendran, A., van der Kaay, J., Poli, V., Cohen, P. \& Alessi, D. R., (2000) The role of 3-phosphoinositide-dependent protein kinase 1 in activating AGC kinases defined in embryonic stem cells. Curr Biol 10: 439-448.

Xu, S., Huang, H. K., Kaiser, P., Latterich, M. \& Hunter, T., (2000) Phosphorylation and spindle pole body localization of the Cdc15p mitotic regulatory protein kinase in budding yeast. Curr Biol 10: 329-332.

Xu, T., Wang, W., Zhang, S., Stewart, R. A. \& Yu, W., (1995) Identifying tumor suppressors in genetic mosaics: the Drosophila lats gene encodes a putative protein kinase. Development 121: 1053-1063. 
Yang, J., Cron, P., Thompson, V., Good, V. M., Hess, D., Hemmings, B. A. \& Barford, D., (2002) Molecular mechanism for the regulation of protein kinase B/Akt by hydrophobic motif phosphorylation. Mol Cell 9: 1227-1240.

Yarden, O., Plamann, M., Ebbole, D. J. \& Yanofsky, C., (1992) cot-1, a gene required for hyphal elongation in Neurospora crassa, encodes a protein kinase. EMBO J 11: 2159-2166.

Yin, F. \& Pan, D., (2007) Fat flies expanded the hippo pathway: a matter of size control. Sci STKE 2007: pe12.

Yoshida, S. \& Toh-e, A., (2001) Regulation of the localization of Dbf2 and mob1 during cell division of Saccharomyces cerevisiae. Genes Genet Syst 76: 141-147.

Zhang, J., Smolen, G. A. \& Haber, D. A., (2008) Negative regulation of YAP by LATS1 underscores evolutionary conservation of the Drosophila Hippo pathway. Cancer Res 68: 2789-2794.

Zhao, B., Wei, X., Li, W., Udan, R. S., Yang, Q., Kim, J., Xie, J., Ikenoue, T., Yu, J., Li, L., Zheng, P., Ye, K., Chinnaiyan, A., Halder, G., Lai, Z. C. \& Guan, K. L., (2007) Inactivation of YAP oncoprotein by the Hippo pathway is involved in cell contact inhibition and tissue growth control. Genes Dev 21: 2747-2761. 


\title{
CHAPTER II
}

\section{The Ndr kinase COT1, and the MAP kinases MAK1 and MAK2 genetically interact to regulate filamentous growth, hyphal fusion and sexual development in Neurospora crassa}

\begin{abstract}
Ndr kinases, such as Neurospora crassa COT1, are important for cell differentiation and polar morphogenesis, yet their input signals as well as their integration into a cellular signaling context are still elusive. Here, we identify the cot-1 suppressor gul-4 as mak-2 and show that mutants of the gul-4/mak-2 mitogen-activated protein (MAP) kinase pathway suppress cot-1 phenotypes along with a concomitant reduction in protein kinase $A$ (PKA) activity. Furthermore, mak-2 pathway defects are partially overcome in a cot-1 background and are associated with increased MAK1 MAPK signaling. A comparative characterization of $N$. crassa MAPKs revealed that they act as three distinct modules during vegetative growth and asexual development. In addition, common functions of MAK1 and MAK2 signaling during maintenance of cell wall integrity distinguished the two ERK-type pathways from the p38type OS2 osmosensing pathway. In contrast to separate functions during vegetative growth, the concerted activity of the three MAPK pathways is essential for cell fusion and the subsequent formation of multicellular structures that are required for sexual development. Taken together, our data indicate a functional link between COT1 and MAPK signaling in regulating filamentous growth, hyphal fusion and sexual development.
\end{abstract}




\section{Introduction}

Apical tip extension is the hallmark of filamentous fungi, and fungal hyphae share, along with neurons and pollen tubes, the distinction of being amongst the most highly polarized cells found (Borkovich et al., 2004, Harris, 2006, Palanivelu \& Preuss, 2000). Polarized growth is a complex multifactorial property, which is coordinated by numerous signals. These pathways, such as the cAMP dependent protein kinase (PKA), the mitogen-activated protein kinase (MAPK) or the nuclear Dbf2-related (Ndr) kinase pathways, are highly conserved and regulate numerous aspects of growth and development including cell proliferation, differentiation, motility and survival, among many others (Hergovich et al., 2006, Lengeler et al., 2000, Lewis et al., 1998). In fungal systems they are important for maintaining polarity, pathogenicity and development (Xu, 2000, D'Souza \& Heitman, 2001, Monge et al., 2006, Xu et al., 2007).

MAPKs are modular signaling units composed of three-tiered kinase cascades, in which a series of three protein kinases phosphorylate and activate one another (Qi \& Elion, 2005). Frequently, a fourth kinase of the Ste20/PAK group acts upstream of the MAPK signaling pathways (therefore also called MAPKKKK (Dan et al., 2001)). Numerous reports have revealed that distinct MAPK pathways are tightly regulated by cross-communication with each other and other signaling pathways (summarized in (Lengeler et al., 2000, Stork \& Schmitt, 2002). Both, the functional modules of each MAPK pathway as well as the interplay between the different signaling routes are best understood in the unicellular ascomycete Saccharomyces cerevisiae and summarized in several recent reviews (Lengeler et al., 2000, Madhani \& Fink, 1998, Pan et al., 2000, Bahn et al., 2007). In the budding yeast, the MAPKs constitute five partially overlapping pathways regulating mating, filamentation, cell integrity, response to high osmolarity and ascospore formation.

In filamentous fungi that undergo highly complex and multicellular developmental phases (e.g. Neurospora crassa has been shown to differentiate into at least 28 different cell types (Bistis et al., 2003)), the situation is much less clear. Three basic MAPK modules have been identified, but, so far, only the kinase cascade homologous to the $S$. cerevisiae osmosensing/stress pathway has been fully characterized in the filamentous ascomycetes $N$. crassa and Aspergillus nidulans (Fujimura et al., 2003, Noguchi et al., 2007, Zhang et al., 2002, Jones et al., 2007). 
Osmostress signaling in N. crassa is transduced through the OS1 histidine kinase to the OS4, OS5 and OS2 MAPK cascade. os mutants are unable to grow on high osmolarity media and are resistant to phenylpyrrole fungicides. Furthermore, lysis and increased pigmentation of asexually derived spores (macroconidia) and female sterility due to the lack of protoperithecia has been reported, yet the cellular or developmental defects involved have not been analyzed in depth (Fujimura et al., 2003, Noguchi et al., 2007, Zhang et al., 2002, Jones et al., 2007). A. nidulans HOG pathway mutants are similarly growth-inhibited under high osmolarity conditions and are sensitive to oxidative stress (Furukawa et al., 2005, Kawasaki et al., 2002). In contrast to the yeast HOG pathway, which depends on two upstream osmosensing branches (the $\operatorname{SIn} 1 \mathrm{p}$ transmembrane hybrid-type histidine kinase and a putative seven transmembrane osmosensor kinase (Maeda et al., 1995, Posas \& Saito, 1998)), activation of this pathway in A. nidulans and N. crassa depends solely on the two component signaling system (Furukawa et al., 2005, Noguchi et al., 2007).

Several MAPK components homologous to the yeast pheromone/filamentation pathway have been found in N. crassa. The MAPKKK NRC1 was first identified as a repressor of the conidiation program, but was later shown to be also involved in hyphal fusion and in the activation of the MAPK MAK2 (Kothe \& Free, 1998, Li et al., 2005, Pandey et al., 2004). Mutants in mak-2 and pp-1 (the downstream transcription factor homologous to yeast Ste12p that is activated by the MAPK Fus3p/Kss1p) display reduced growth rates, the inability to undergo hyphal fusion, shortened aerial hyphae formation and de-repressed conidiation. Furthermore, they fail to develop protoperithecia, and ascospores carrying null mutations of either gene are autonomous lethal (Li et al., 2005, Pandey et al., 2004). A similar pleiotrophic phenotype has been observed in SteC MAPKKK mutants in A. nidulans, which result in slower growth rates, more branched hyphae, altered conidiophore morphology, inhibition of heterokaryon formation and inhibited sexual development (Wei et al., 2003). Additional homologues of budding yeast Fus3p/Kss1p have been characterized in several pathogenic fungi and have been shown to play key roles in appressorium formation and host colonization (Xu, 2000).

Even though mutants in the MAPK homologous to yeast Slt2 have been generated in $A$. nidulans, and in several phytopathogenic fungi (Bussink \& Osmani, 1999, Xu et al., 1998, Kojima et al., 2002, Mey et al., 2002, Hou et al., 2002), information concerning this third MAPK pathway in filamentous fungi is still limited. Common phenotypes of SIt2-like kinase 
mutants included altered cell walls and defects in conidial germination (which could be remedied by high osmolarity media) and autolysis in central areas of the colony, suggesting the involvement of a cell integrity-type MAPK pathway in filamentous fungi. Furthermore, the Fusarium graminearum SIt2 homolog MGV1 is required for female fertility, heterokaryon formation and plant infection (Hou et al., 2002).

The functional analysis of $\mathrm{Ndr}$ kinases has gained much interest in recent years. They are important for normal cell differentiation and polar morphogenesis in various organisms, yet their specific functions are still elusive (Geng et al., 2000, Racki et al., 2000, Yarden et al., 1992, Zallen et al., 2000) summarized in (Hergovich et al., 2006). An interesting connection between Ste20/PAK (= MAPKKKK) and Ndr kinase signaling was provided through the analysis of the Schizosaccharomyces pombe Ndr kinase mutant orb-6 (Verde et al., 1998). orb- 6 and pak-1 share similar phenotypes, double mutants are synthetically lethal, and the over expression of ORB6 in pak-1 partially suppressed the pak-1 defect, suggesting that PAK1 acts upstream of ORB6. Furthermore, members of the MST2 and MST3 groups of Ste20 kinases have recently been described as upstream regulators of $\mathrm{Ndr}$ kinases (Emoto et al., 2006, Kanai et al., 2005, Nelson et al., 2003, Stegert et al., 2005).

The MST3 and Ndr kinases POD6 and COT1 of N. crassa are essential for hyphal tip extension and coordinated branch formation. Both kinases have been shown to interact, they share common suppressors and are localized in a kinesin/dynein-dependent manner (Seiler et al., 2006). We have provided evidence indicating that COT1/POD6 and PKA act in parallel pathways that regulate polarity formation in a positive or negative manner, respectively, in N. crassa (SEILER et al., 2006). However, the input and outcome components of the Ndr kinase network as well as its integration into a cellular signaling context have not been described in any system. This information is critical for elucidation of the mechanistic involvement of $\mathrm{Ndr}$ kinases in cell growth and polarity.

The described differences between the MAPK pathways in various filamentous fungi and yeasts highlight the need for a comparative analysis of MAPK modules during vegetative growth and the multiple developmental decisions made in a filamentous fungus. Here, we describe three MAPK cascades, which function as distinct modules during vegetative growth of $N$. crassa, but their joint activity is necessary for hyphal fusion and the development of complex multicellular sexual structures. Furthermore, we provide evidence for cross-talk between COT1 and the MAK1 and MAK2 pathways. 


\section{Results}

\section{Mutants of the MAK2 MAP kinase pathway suppress cot-1 growth defects}

The phenotypic characteristics of the conditional cot-1(ts) mutant, which forms tight colonies with growth-arrested needle-shaped hyphal tips when germinated at restrictive temperature, facilitates the easy identification of cot-1 suppressors. This efficient procedure makes N. crassa ideal for the genetic dissection of Ndr signaling. Several mutants designated gulliver that act as modifiers of the compact cot-1(ts) morphology at restrictive temperature have been described (Bruno et al., 1996b, Seiler et al., 2006, Terenzi \& Reissig, 1967). gul-4 has been mapped to nic-3 (17\%) on linkage group VII (Perkins et al., 2001). Using additional auxotropic markers, we determined that gul-4 is closely linked with arg-10, arg-11 and met$7(<1 \%,<1 \%$, and $<0,1 \%$ recombination frequencies, respectively). This information and the available genome sequence identified several candidate genes for gul-4. By sequencing potential ORFs as well as their 5' and 3' untranslated regions, we identified a 12 bp insertion (CAA CAA CAA CAA) in the mak-2 promotor at position -270/271 upstream of the start ATG as a potential cause for the suppression of cot-1(ts). To test if gul-4 is allelic to mak-2, we generated a cot-1(ts); $\Delta m a k-2$ double mutant. When tested at restrictive temperature, the $\Delta$ mak-2 deletion partially suppressed the cot-1(ts) defect in a manner identical to that observed in the original gul-4 background (Figure II-1 A; Southern blot analyses confirming the genetic nature of the double mutants generated throughout this report are available as Supplementary Figure S II-1). Microscopic analysis of the hyphal apex revealed that in contrast to the extension-arrested pointed tips of cot-1(ts) grown at restrictive temperature, the cot-1(ts);gul-4 and cot-1(ts);Amak-2 strains generated a dome shaped apex, typical of a normal (although slow) growing tip (Figure II-1 B). The presence of a tight genetic linkage between mak-2 and gul-4 was made evident by the analysis of crosses between cot$1(t s) ; g u l-4$ and cot-1; $\Delta m a k-2$. Out of $>2,000$ progeny screened, no cot-1(ts);gul ${ }^{+}$strains were obtained. To confirm that gul-4 is allelic to mak-2, we expressed MAK2 in gul-4 and $\Delta m a k-2$ and found that it complemented the growth defects of both mutants (data not shown). Furthermore, when we expressed MAK2 in cot-1(ts);gul-4 and cot-1(ts);Amak-2, the suppression of the cot-1(ts) growth defect was abolished at the restrictive temperature (Figure II-1 C). 


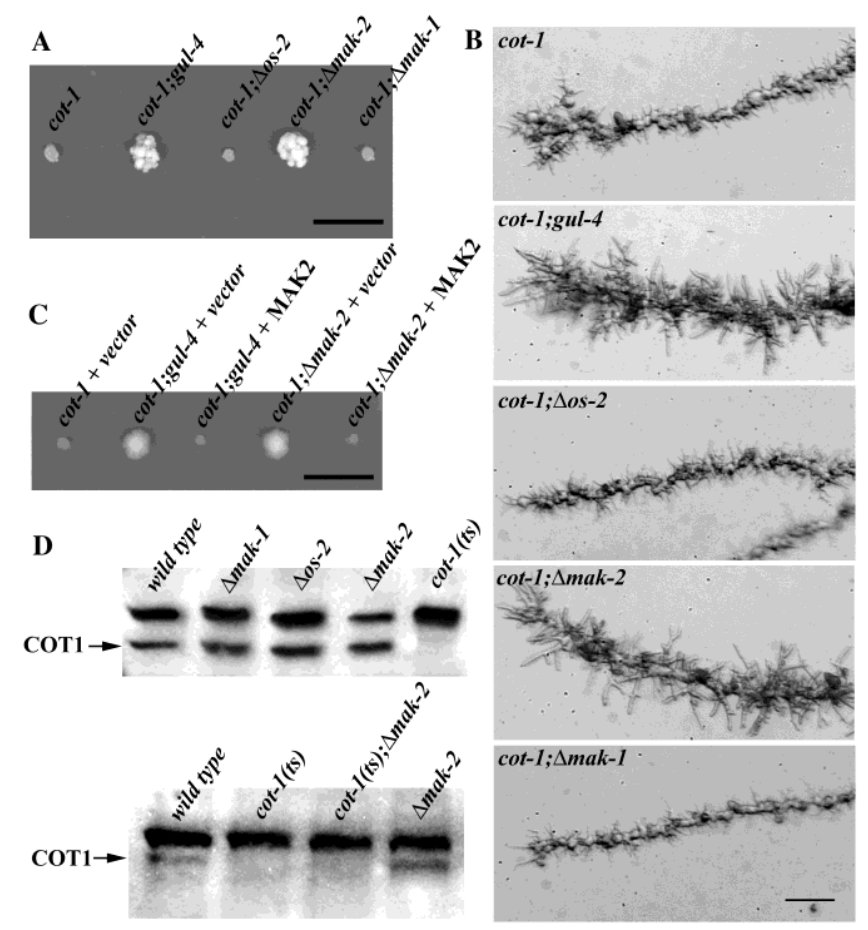

Figure II-1 gul-4/Amak-2 strains suppress the cot-1(ts) growth defects. (A) The indicated strains were germinated and grown on minimal media plates for 3 days at $37^{\circ} \mathrm{C}$. Note the increased colony diameters of cot1(ts);gul-4 and cot-1(ts); $\Delta$ mak-2 compared to cot-1(ts). Bar $=1 \mathrm{~cm}$. (B) Results of temperature shift experiments, in which strains grown at $25^{\circ} \mathrm{C}$ and shifted to $37^{\circ} \mathrm{C}$ for $8 \mathrm{~h}$ illustrate pointed growth-arrested tips of cot-1(ts), cot-1(ts);Aos-2, and cot-1(ts); $\Delta m a k-1$, while dome shaped slow-growing apices are visible in cot1(ts),gul-4 and cot-1(ts);Amak-2. Bar $=20 \mu \mathrm{m}$. (C) The indicated strains were transformed with mak-2 expression vector or the empty vector as control and grown on minimal media plates supplemented with 30 $\mu \mathrm{g} / \mathrm{ml}$ nourseothicin for 3 days at $37^{\circ} \mathrm{C}$. Bar $=1 \mathrm{~cm}$. (D) Western-Blot analysis of cell extracts probed with antiCOT1 antibodies indicate that deleting any of the three MAPKs does not affect COT1 expression (upper panel) and that the gulliver-like suppression of the cot-1(1) phenotype by $\Delta$ mak-2 at restrictive temperature is independent of the presence of the COT1 $67 \mathrm{kDa}$ band (arrow on lower panel).

To determine if the suppression of cot-1(ts) is specific to the MAK2 MAPK pathway, we generated double mutants of cot-1(ts) with loss of function mutants in os-2 and mak-1, the other two MAPK genes present in the $N$. crassa genome (Borkovich et al. 2004). When we introduced the three MAPK mutations into the cot-1(ts) background, only $\Delta m a k-2$ suppressed the cot-1(ts) growth defects, indicating a specific interaction between COT1 and MAK2 kinase signaling (Figure II-1 A, B).

Western analyses were performed in order to determine if deletion of any one of the three MAPKs affected the pattern of COT1 expression (Figure II-1 D). The typical 67 kDa COT1 band was clearly evident in protein extracts of all three MAPK mutants. Furthermore, loss of 
MAK2 function in cot-1(ts); $\Delta m a k-2$ did not confer quantitative or qualitative alterations in the COT1 protein expression pattern, indicating that the improved growth of $\cot -1$ (ts) by deleting mak-2 was not dependent on the presence of COT1. Based on these results, we concluded that COT1 and MAK2 act in independent pathways, and that the suppression of the cot-1(ts) defect was indirect.

\section{Deletion of mak2 is accompanied by a reduction in PKA activity}

The suppression of cot-1(ts) by $\Delta m a k-2$ resembled the previously described environmental suppression of cot-1(ts) and pod-6(ts) by external stresses (Gorovits \& Yarden, 2003, Seiler et al., 2006). As environmental suppression of both kinases was correlated with reduced PKA activity levels, we analyzed PKA activity in the $\Delta$ mak-2 strain and found several lines of evidence for reduced PKA activity. $m c b$ is a temperature-sensitive mutant defective in the regulatory subunit of PKA, which displays elevated PKA activity levels at restrictive temperature, resulting in apolar growth and irregular chains of spherical cells (Bruno et al., 1996a, Seiler et al., 2006, Ziv et al., 2007, Ziv et al., 2008). Genetic analysis of a Amak$2 ; m c b(14-4)$ double mutant demonstrated that $\Delta m a k-2 ; m c b(14-4)$ grew slower than the parental strains at permissive temperature, suggesting a genetic interaction between MAK2 and PKA signaling. Nevertheless, the $\Delta m a k-2$ background partially suppressed the polarity defect of $m c b$ (14-4) at restrictive temperature, suggesting that PKA activity levels are reduced in $\Delta$ mak-2 (Figure II-2 A). To test this hypothesis, we increased the cellular PKA activity in cot-1(ts); $\Delta m a k-2$ grown at $37^{\circ} \mathrm{C}$ by culturing the strain in the presence of $500 \mu \mathrm{M}$ 8-Br-cAMP, which mimics increased levels of cAMP, and found that the suppressive effect of $\Delta m a k-2$ on cot-1(ts) at restrictive temperature was abolished (Figure II-2 B), while it had only a minor affect on the growth rate of cot-1(ts) or wild type (data not shown). Finally, we directly measured PKA activity in $\Delta m a k-2$ single and $\Delta m a k-2 ; \cot -1(t s)$ double mutants and found that a significant $(P<0.001$; paired two sample $t-T e s t)$ reduction in PKA activity could be detected in these strains (Figure II-2 C). Several measurements (with independent cultures) detected a consistent $30-35 \%$ decrease in kinase activity in the $\Delta m a k-2$ and cot1(ts); Amak-2 strains in comparison to wild type. A $70 \%$ increase $(\mathrm{P}<0.001)$ in PKA activity was measured in the $m c b(14-4)$ control, as expected (ZIV et al., 2008). Thus, we suggest that 
the suppression of cot-1 by the deletion of mak-2 is part of a bypass mechanism, which includes a reduction in PKA activity levels.
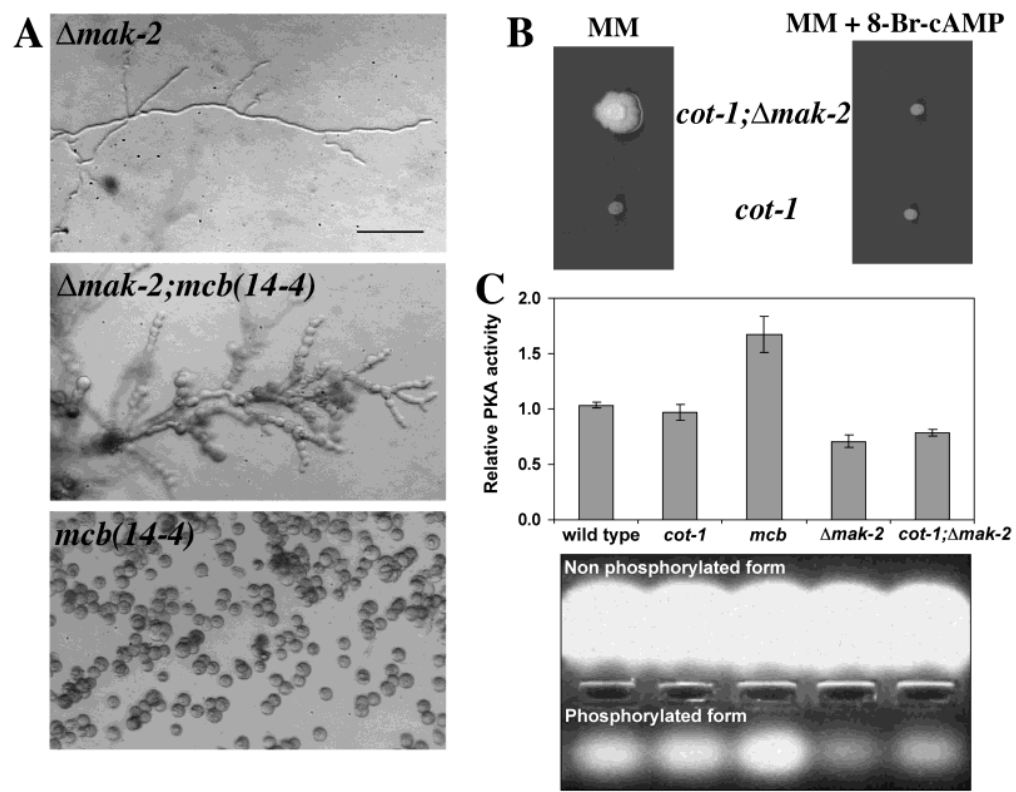

Figure II-2 PKA activity is reduced in $\Delta m a k-2$. (A) Morphology of $\Delta m a k-2 ; m c b(14-4)$, and $m c b(14-4) ; \Delta m a k-2$ germinated for $12 \mathrm{~h}$ at $37^{\circ} \mathrm{C}$. Bar $=20 \mu \mathrm{m}$. (B) Growth of cot-1(ts) and cot-1(ts); $\Delta m a k-2$ on minimal media and media supplemented with $500 \mu \mathrm{M}$ 8-Br-cAMP at restrictive temerature. (C) PKA activity in extracts of germinating conidia of wild type, cot-1(ts), mcb(14-4), $\Delta m a k-2$ and cot-1(ts); $\Delta m a k-2,11 \mathrm{~h}$ post-inoculation, relative to wild type. Cultures were incubated in pre-warmed liquid Vogel's minimal at $36^{\circ} \mathrm{C}$ and were assayed for PKA activity. Data presented in the chart are means of at least four independent experiments with two replicates each. Standard errors are shown. The bottom figure presents a selected experiment demonstrating the nonphosphorylated and phosphorylated (indicating PKA activity) fluorescent Kemptide substrates, migrated to the anode and cathode of the agarose gel, respectively. The PepTag ${ }^{\circledR}$ Assays utilize fluorescent peptide substrates specific for PKA. Phosphorylation of the substrate by PKA alters the peptide's net charge from +1 to -1 , allowing separation of the phosphorylated substrate from the non-phosphorylated on the agarose gel.

\section{The three $\boldsymbol{N}$. crassa MAP kinases act as three distinct modules during growth and development}

To further dissect the cross-communication between the MAK2 pathway and COT1 signaling, we carried out a comparative characterization of the three N. crassa MAPKs (Figure II-3). Dos-2 displayed growth rates that were similar to wild type, but frequent dichotomous 
branching events suggested minor defects at the hyphal apex. The conidial lysis defect and its sensitivity to sorbitol have already been described (Noguchi et al., 2007, Zhang et al., 2002), and these phenotypes clearly distinguished $\Delta o s-2$ from $\Delta m a k-1$ and $\Delta m a k-2 . \Delta m a k-1$ was the most drastically growth-impaired MAPK mutant with tip extension rates of less than $15 \%$ of wild type forming a rosetta-like colony (Figure II-3 A). The mutant was almost devoid of aerial hyphae, produced few conidia and the conidial population was highly enriched with arthroconidia ( $86 \%$ compared with $5 \%$ in wild type). Abnormal and apolar branching events indicated a major defect during polarity establishment of newly formed branches. Polarity establishment was also affected during germination. Only $15 \%$ of $\Delta m a k-1$ conidia produced germ tubes after $7 \mathrm{~h}$ in liquid minimal medium in contrast to $85 \%$ of wild type conidia. $\Delta m a k-1$ and $\Delta m a k-2$ exhibited a cell wall defect, as protoplast production was $\sim 4$ and 2 -fold, respectively, enhanced in comparison to wild type in the presence of Novozyme. In addition, their growth behavior on plates containing $1 \%$ sucrose and $1 \%$ sorbose in the presence of a concentration gradient of lysing enzymes indicated that both $\Delta m a k-1$ and $\Delta m a k-2$ have altered cell walls, with $\Delta m a k-1$ being more sensitive than $\Delta m a k-2$ (Figure II-3 B; note that the effect of sorbose on tip extension and the cell wall is not compensated by the addition of lysing enzymes in a manner similar to the wild type and $\Delta o s-2$ strains). Besides their common cell wall defect, the two strains displayed additional similarities such as their conidial sensitivity to high temperature, which could be overcome by the addition of $1 \mathrm{M}$ sorbitol prior to the heat shock. In addition, $\Delta m a k-1$ is sensitive to formamide, a general stressinducing agent, which is readily taken up by fungi yet is not metabolized (Hampsey, 1997). A unique defect of $\Delta m a k-2$ is its highly irregular zig-zagging growth, which suggested Spitzenkörper positioning defects, but no altered sensitivity to the microtubule inhibitors Benomyl or Nocodazol were observed (data not shown).

Amak-2 has been described as female sterile (Li et al., 2005, Pandey et al., 2004), but the exact developmental block in sexual development has not been reported. Inspection of $\Delta m a k-2$ grown on cornmeal agar plates for 10 days revealed no mature and fertilizationcompetent protoperithecia (female sexual structures in $N$. crassa), but a ca. 50 -fold reduced number of protoperithecia-like structures in comparison to wild type (Figure II-3 A). Furthermore, the protoperithecia-like structures produced in the $\Delta m a k-2$ strain were smaller, less developed and non-fertile, but morphologically resembled immature protoperithecia of wild type (e.g. Poggeler and Kuck 2004; Poggeler et al. 2006). This 
indicated that loss of the MAK2 pathway function does not abolish the capability to initiate protoperithecia formation, but rather affects their abundance and, more importantly, their maturation into fertile structures. Interestingly, when we tested $\Delta o s-2$ and $\Delta m a k-1$, we found them to also be female sterile yet they produced no protoperithecia at all. Thus, the other two MAPK mutants were blocked at an earlier developmental stage. In $\Delta m a k-1$, we observed only lasso-like structures embedded in the agar, suggesting failed attempts of hyphae to coil and fuse during ascogonia formation. In $\Delta o s-2$, we detected the presence of small, curled side branches, typical of early stages during ascogonia formation suggesting that both strains are blocked at, or even prior to, the initiation of ascogonia formation.
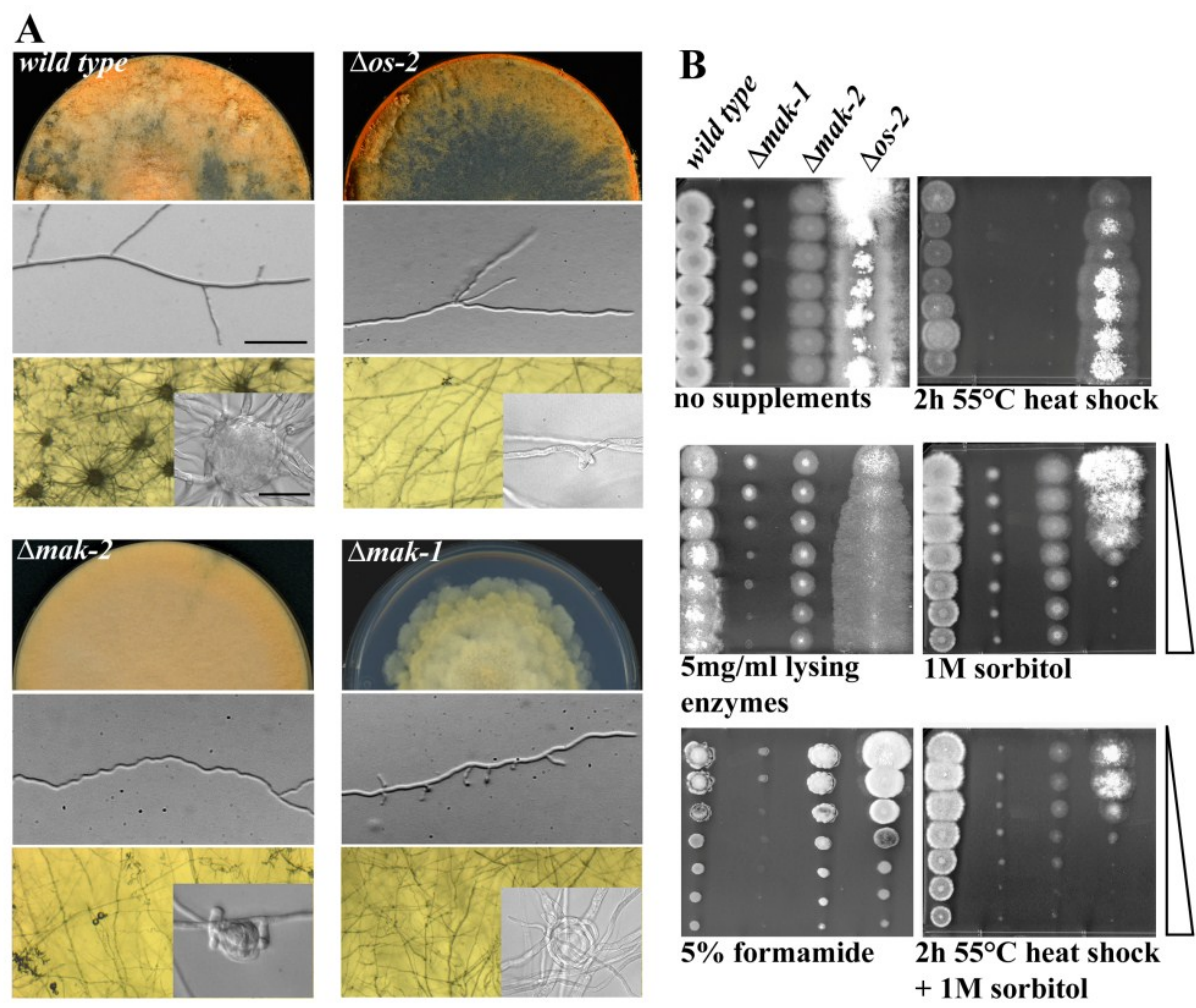

Figure II-3 Comparative characterization of the N. crassa MAP kinase mutants. (A) Colony morphology, asexual development and hyphal morphology (upper and middle panel, respectively, bar $=20 \mu \mathrm{m}$ ) of the indicated strains grown on minimal media plates is shown. Sexual development (lower panel) was induced by growth for 5 days on Cornmeal agar. The inserts illustrate the terminal morphology of the female reproductive structures (protoperithecia). Bar $=10 \mu \mathrm{m}$. (B) Growth of the three MAPK mutants on gradient plates supplemented with the indicated additives. $5 \times 10^{3}$ conidia were inoculated for each spot. Wedges denote the compound gradient. To restrict the radial growth rates of the strains, all plates were supplemented with $1 \%$ sorbose in addition to the indicated additives. 
To better characterize the modularity of the upstream MAPKs (Borkovich et al., 2004, Galagan et al., 2003), we extended this analysis to include the respective MAPKK and MAPKKK components. Three distinct MAP kinase cascades were previously found by in silico analyses in several fungal genomes (Borkovich et al., 2004, Galagan et al., 2003) but a comparative functional characterization is still lacking. Several of the mutants provided by the genome project (Dunlap et al., 2007) were only available as heterokaryons and were therefore backcrossed to wild type to isolate homokaryotic deletions or, if crosses were not successful, the heterokaryons were colony-purified several times and their homokaryotic status confirmed by Southern analysis (Table II-2). A detailed phenotypic analysis of the mutants confirmed the phylogenetic comparison and supported the existence of three functional modules (Table II-1), each consisting of a kinase, a kinase-kinase and a kinasekinase-kinase, which each displayed identical phenotypes, based on growth rate, hyphal morphology, conidiation pattern, sexual development, and behavior with respect to inhibitors. The only exception was $\Delta m i k-1$, which displayed a slightly better growth rate and produced more conidia than the respective MAPKK and MAPK mutants of the MAK1 pathway. Furthermore, double mutant analysis of $\cot -1$ (ts) with the available MAPKK and MAPKKK mutants corroborated that the suppression of the cot-1 defect was specific for mak-2 pathway deletions (Table II-1). 
Table II-1 Summary of phenotypic charateristics of $N$. crassa MAPK pathway single and respective cot-1;MAPK double mutants.

\begin{tabular}{|c|c|c|c|c|c|c|c|c|}
\hline \multirow[b]{2}{*}{ Strain } & \multicolumn{2}{|c|}{ Growth rates $^{1}$} & \multicolumn{2}{|c|}{ Vegetative fusion } & \multirow{2}{*}{$\begin{array}{c}\text { Major hyphal defects of } \\
\text { MAP kinase mutants }\end{array}$} & \multirow{2}{*}{\begin{tabular}{|c|} 
Asexual development of \\
MAP kinase mutants \\
\end{tabular}} & \multicolumn{2}{|c|}{ Female fertility $^{3}$} \\
\hline & mapk & cot-1(ts);mapk & mapk & cot-1(ts);mapk & & & mapk & cot-1(ts);mapk \\
\hline wild type & & 3.5 & & yes & l & I & & yes \\
\hline $\cot -1(t s)$ & & 3.2 & & yes & / & / & & yes \\
\hline $\begin{array}{r}\text { Osmosensing pathway } \\
\Delta o s-4 \text { (NCU03071) } \\
\Delta o s-5 \text { (NCU00587) } \\
\Delta o s-2 \text { (NCU07024) }\end{array}$ & $\begin{array}{l}3.2 \\
3.1 \\
3.2\end{array}$ & $\begin{array}{l}\mathrm{nd}^{4} \\
3.0 \\
3.2\end{array}$ & $\begin{array}{l}\text { no } \\
\text { no } \\
\text { no }\end{array}$ & $\begin{array}{l}\text { nd }^{4} \\
\text { no } \\
\text { no }\end{array}$ & $\begin{array}{l}\text { +/- wild type; } \\
\text { frequent tip splitting }\end{array}$ & conidial lysis & $\begin{array}{l}\text { no } \\
\text { no } \\
\text { no }\end{array}$ & $\begin{array}{l}\mathrm{nd}^{4} \\
\text { no } \\
\text { no }\end{array}$ \\
\hline $\begin{array}{c}\text { Cell fusion / fertility pathway } \\
\text { Anrc-1 (NCU06182) } \\
\Delta m e k-2 \text { (NCU04612) } \\
\Delta m a k-2 \text { (NCU02393) }\end{array}$ & $\begin{array}{l}1.2 \\
1.1 \\
1.2\end{array}$ & $\begin{array}{l}2.5 \\
\mathrm{nd}^{4} \\
2.6\end{array}$ & $\begin{array}{l}\text { no } \\
\text { no } \\
\text { no }\end{array}$ & $\begin{array}{l}\text { yes } \\
\mathrm{nd}^{4} \\
\text { yes }\end{array}$ & $\begin{array}{l}\text { higly irregular growth } \\
\text { axis }^{2}\end{array}$ & $\begin{array}{l}\text { reduced aerial hyphae } \\
\text { and conidia formation }{ }^{2}\end{array}$ & $\begin{array}{l}\text { no } \\
\text { no } \\
\text { no }\end{array}$ & $\begin{array}{l}\text { yes } \\
\mathrm{nd}^{4} \\
\text { yes }\end{array}$ \\
\hline $\begin{array}{c}\text { Cell wall integrity pathway } \\
\Delta m i k-1 \text { (NCU02234) } \\
\Delta m e k-1 \text { (NCU06419) } \\
\Delta m a k-1 \text { (NCU11376) }\end{array}$ & $\begin{array}{l}0.6 \\
0.5 \\
0.6\end{array}$ & $\begin{array}{l}\mathrm{nd}^{4} \\
0.6 \\
0.5\end{array}$ & $\begin{array}{l}\text { no } \\
\text { no } \\
\text { no }\end{array}$ & $\begin{array}{l}\text { nd }^{4} \\
\text { no } \\
\text { no }\end{array}$ & $\begin{array}{l}\text { polarity defect; } \\
\text { branch formation } \\
\text { abnormal }\end{array}$ & $\begin{array}{l}\text { arthroconidiation for } \\
\text { mak-1 and mek-1 }\end{array}$ & $\begin{array}{l}\text { no } \\
\text { no } \\
\text { no }\end{array}$ & $\begin{array}{l}\text { nd }^{4} \\
\text { no } \\
\text { no }\end{array}$ \\
\hline
\end{tabular}

${ }^{1}$ in $\mathrm{cm} /$ day at $20^{\circ} \mathrm{C}(\mathrm{n}=3)$ as determined by radial hyphal growth experiments.

${ }^{2}$ no differences in hyphal morphology and asexual development was observed for the single and respective cot-1 double mutants grown at $25^{\circ} \mathrm{C}$ exept for a better condidation rate of the cot-1;mak-2 and cot-1;nrc-1 double mutants compared to mak-2 and $n r c-1$.

${ }^{3}$ protoperithecia formation after 7 days at RT on $2 \%$ cornmeal agar supplemented with $0,1 \%$ glucose and viable ascospore formation when fertilized with wild type conidia.

${ }^{4}$ not determined, as we were not able to obtain viable hygromycin-resistent ascospores in crosses with wild type or cot-1 as female partner 
An increase in MAK1 activity in a cot-1 background bypasses the mak-2 pathway defects

In a more detailed analysis of the MAPK deletions and in comparison with respective cot1(ts) double mutants, we determined that cot-1(ts); $\Delta m a k-2$ and cot-1(ts); $4 n r c-1$ double mutants had a an intermediate growth rate when compared to that of the parental strains when grown at permissive conditions (Figure II-4 A; Table II-1). Additional mak-2 pathway defects, such as their shortened aerial hyphae, the de-repression of their conidial production and the female sterility, were also suppressed in the cot-1(ts) background (Figure II-4 B, C). As $\Delta m a k-2$ and $\Delta n r c-1$ have been described as hyphal fusion defective mutants (Pandey et al., 2004), we also tested if cot-1(ts) has any effect on the fusion of vegetative hyphae. cot-1(ts) grown at permissive temperature is fusioncompetent, and we did not observe any qualitative differences when compared with wild type. When we analyzed the cot-1(ts); $\Delta m a k-2$ and cot-1(ts); $\Delta n r c-1$ double mutants, we observed a suppression of the fusion defect of the mak-2 pathway deletions (Figure II-5; Table II-1). The resulting interconnected, syncycial mycelium could increase the efficiency of nutrient flow and organelle distribution throughout the colony. This, in turn, may explain the increased growth rate, the enhanced formation of aerial hyphae, the better conidiation rates and the restored female fertility of the double mutants in comparison to the mak-2 pathway deletions. 

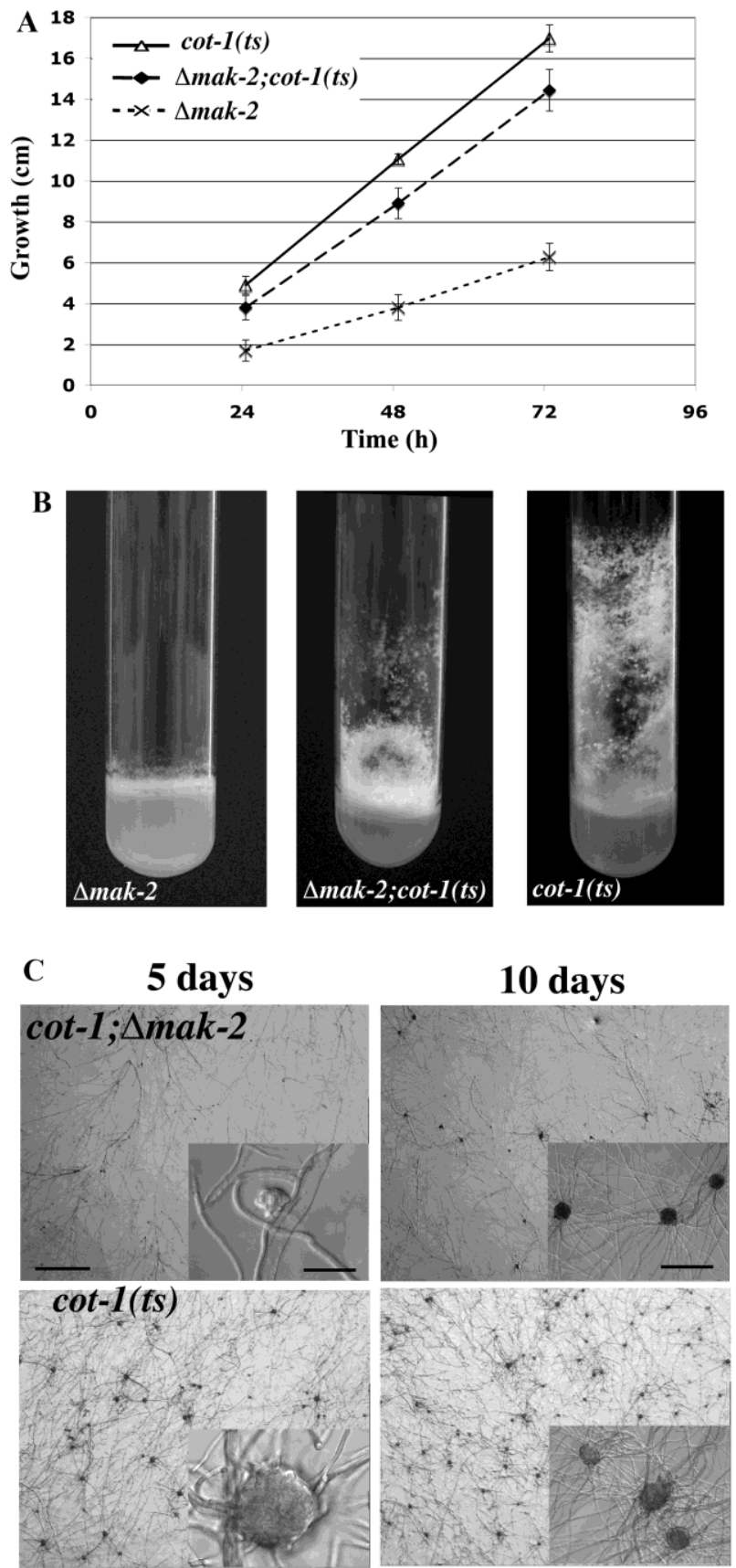

Figure II-4 mak-2 pathway defects are suppressed when COT1 activity is reduced. (A) cot-1(ts); $\Delta$ mak-2 grown at $25^{\circ} \mathrm{C}$ in race tubes has an intermediate tip extension rate $(\mathrm{A})$ and generates intermediate amounts of aerial hyphae and conidia (B) when compared to the parental strains. (C) Time course of protoperithecia formation by cot-1(ts); $\Delta$ mak-2. Bars $=100 \mu \mathrm{m}$ ( 5 and 10 days overview), $10 \mu \mathrm{m}$ (5 day insert) and $25 \mu \mathrm{m}$ (10 day insert).

To confirm that this cot-1-dependent suppression is specific for the MAK2 pathway, we analyzed the involvement of the other two MAPK modules in the hyphal fusion process. When we tested $\Delta o s-2$ and $\Delta$ mak-1, we found that both mutants were also defective in

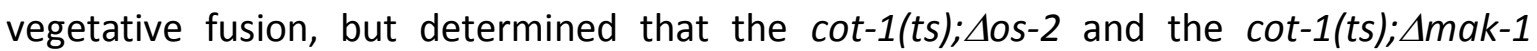


double mutants did not regain their fusion competence (Figure II-5). We also tested the remaining MAPKK and MAPKKK deletions and found them to be fusion-defective. Thus, the $\cot -1$ (ts) dependent suppression was specific for mak-2 pathway components (Table II-1). Taken together, these data indicate that the activity of all three MAPK pathways is essential for hyphal fusion. However, based on the specificity of cot-1(ts) suppression of mak-2 pathway deletion strains, this also indicates the presence of different mechanistic functions of the three MAP pathways during cell fusion.

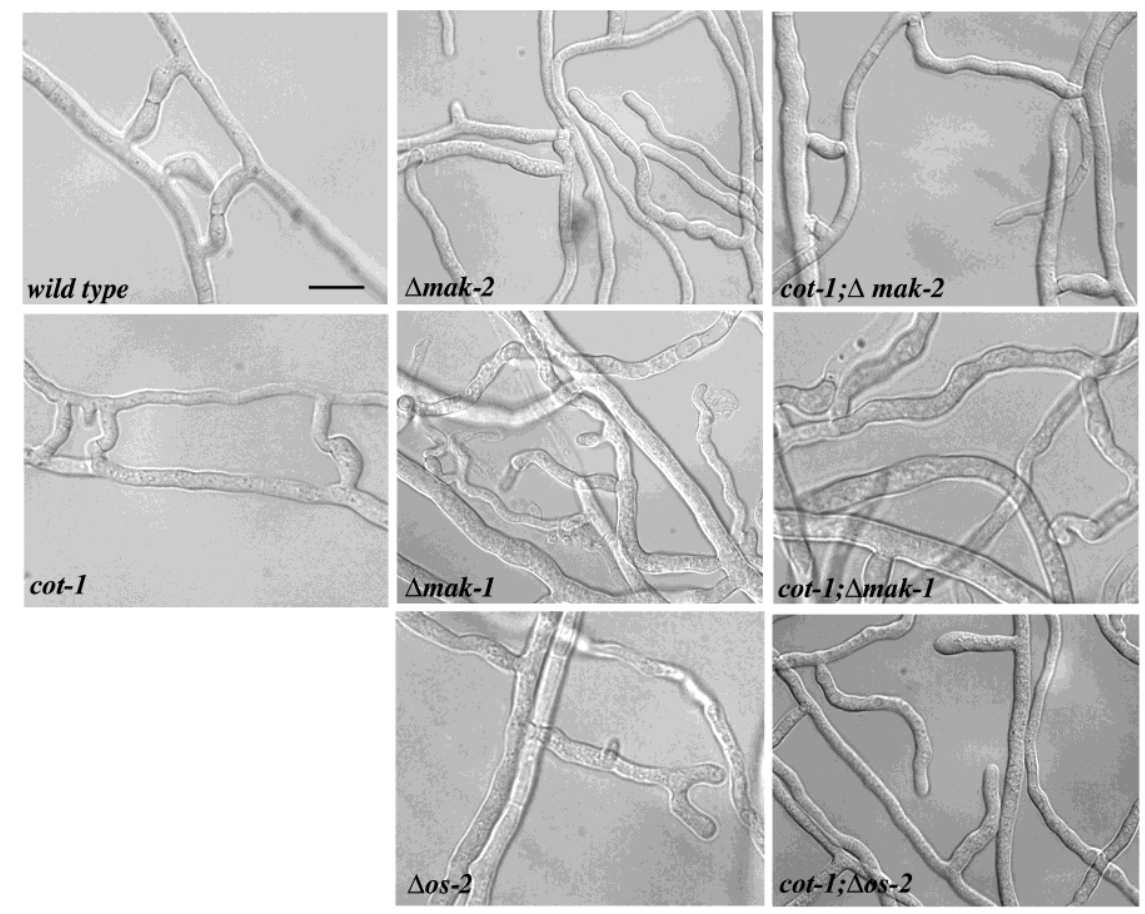

Figure II-5 Hyphal fusion is dependent on the three MAP kinase modules. Microscopic analysis of the indicated strains grown for 2 days on minimal media plates at $25^{\circ} \mathrm{C}$. Note that the three MAPK mutants show extended cell-cell contacts, but no distinct fusion bridges, which are clearly visible in wild type, cot1(ts) and cot-1(ts); $\Delta m a k-2$. Bar $=5 \mu \mathrm{m}$.

The characterization of the MAPK mutants has revealed phenotypic similarities between the mak-1 and mak-2 pathway deletion strains, indicating a potential functional overlap between the two signaling cascades. Therefore, the loss of one pathway may affect the MAPK activity of one or two of the others. We tested the activity of the three MAPKs and found it to increase under various stress conditions, as determined by the use of phospho-specific antibodies against activated MAPKs (Figure II-6 A, upper panel). Nevertheless, we detected a similar phospho-activation pattern of the two remaining MAPK pathways when one MAPK was deleted, suggesting that there is no compensatory 
activation of the other MAPK pathways under normal stress-sensing conditions (Figure II6 A, lower panel). However, in cot-1(ts), we detected a marked increase of MAK1 phosphorylation as measured $8 \mathrm{~h}$ after the shift to restrictive temperatures while MAK2 activity remained constant (Figure II-6 B). As mentioned before, cot-1(ts); $\Delta$ mak-1 pathway double mutants did not display any synthetic characteristics. Thus, these results identified COT1 as a potential negative regulator of MAK1 activity.
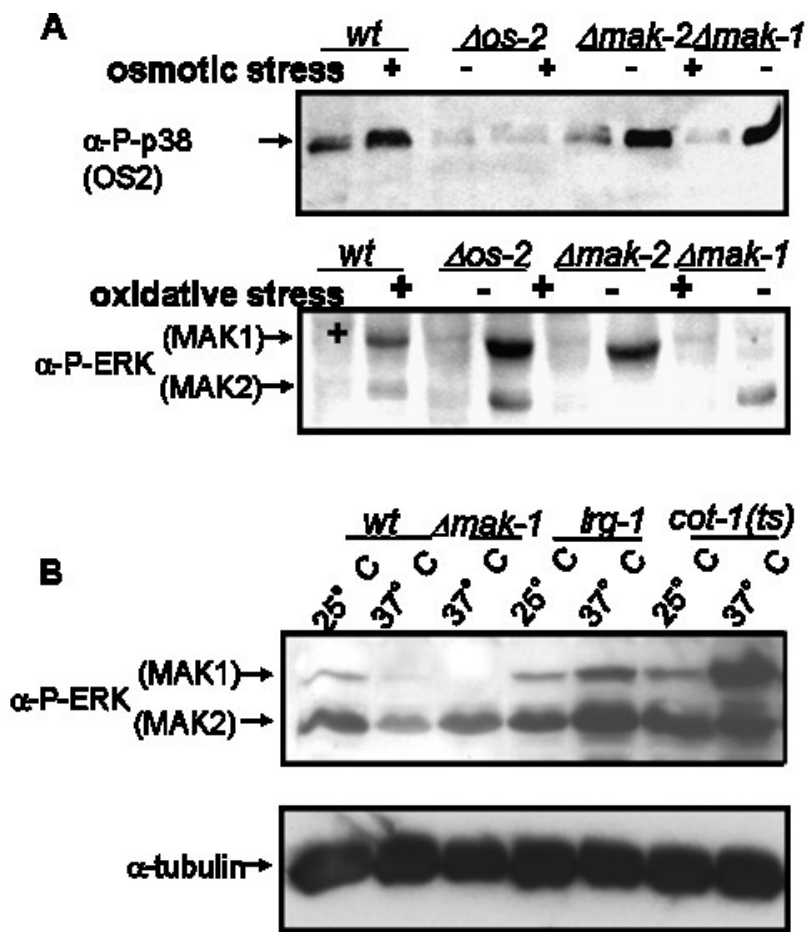

Figure II-6 MAK1 activity is increased in cot-1(ts). (A) Total soluble protein (100 $\mu \mathrm{g}$ per lane) was extracted from the indicated strains grown in the presence or absence of stress-inducers ( $1 \mathrm{M} \mathrm{NaCl} ; 7 \mathrm{mM} \mathrm{H}_{2} \mathrm{O}_{2}$ ). The blot was probed with anti-phospho-ERK ( $\alpha$-P-ERK) and anti-phospho-p38 ( $\alpha$-P-p38) antibodies to detect activated MAK1, MAK2 and OS2 kinase. (B) For the temperature shift experiments, total soluble protein (50 $\mu \mathrm{g}$ per lane) of the indicated strains grown at $25^{\circ} \mathrm{C}$ and shifted to $37^{\circ} \mathrm{C}$ for $12 \mathrm{~h}$ was extracted and the Blot probed with anti-phospho-ERK ( $\alpha$-P-ERK) antibody (upper panel). To confirm equal loading the Blot was stripped and re-probed with $\alpha$-tubulin antibody (lower panel). Irg-1 is an unrelated temperature-sensitive hyperbranching mutant used as a control. 


\section{Discussion}

Molecular understanding of fungal morphogenesis is still a major challenge. Phylogenetic analyses and the comparison of $S$. cerevisiae morphogenetic data with the limited results from various filamentous asco- and basidiomycetes have established that a core set of „polarity factors", including the existence of most signal transduction pathway components, are conserved between unicellular and filamentous fungi (Borkovich et al., 2004). Nevertheless, it is becoming increasingly evident that differences in the wiring of these conserved components and the presence of additional proteins that are absent in unicellular fungi result in dramatically different morphogenetic outcomes that range from unicellular to true filamentous growth and multicellular differentiation.

The recent advent of available genome sequences for several filamentous fungi (Dean et al., 2005, Galagan et al., 2003, Galagan et al., 2005) has provided the MAPK tool-box present in filamentous ascomycetes. In this report, we comparatively characterized the nine components of three MAPK modules of $N$. crassa, and provided evidence that they act as three distinct modules during vegetative growth and asexual development, but also that the joined activity of the three pathways is required for hyphal fusion and for the formation of more complex multicellular structures necessary to undergo sexual development. Furthermore, we suggest a partial overlap of MAK1- and MAK2-dependent signaling for maintaining the functions of the cell wall based on shared phenotypes and similar sensitivities against cell wall drugs, which distinguished the two ERK-type MAPK routes from the p38-type OS2-dependent osmosensing pathway (Figure II-7).

Despite their common phenotype as female sterile mutants, we observed distinct terminal phenotypes of the MAPK mutants during the development of female reproductive structures. Thus, the three MAPK pathways seem to act by different mechanisms in regulating sexual development. The function of the MAK2 pathway was not necessary for the initial steps during the formation of ascogonia, but was required for the maturation of young protoperithecia. In contrast, mutants in the other two pathways are blocked prior to the formation of ascogonia. The coiling of $\Delta m a k-1$ may indicate defects in cell-cell contact formation due to an altered cell wall or may suggest cell-cell signaling defects, while in $\Delta o s-2$ we only observed small, bent side branches, suggesting that even the initial attempts of hyphal curling during ascogonia formation are defective. 
Based on the relative late block in the formation of female reproductive structures in mak-2 pathway deletions, we speculate that the MAK2 pathway is an integral part of sexual development, and that blocking either of the other two pathways impairs the sexual cycle by preventing the initiation of fruiting body development or as part of the pleiotropic consequences of their inactivation.

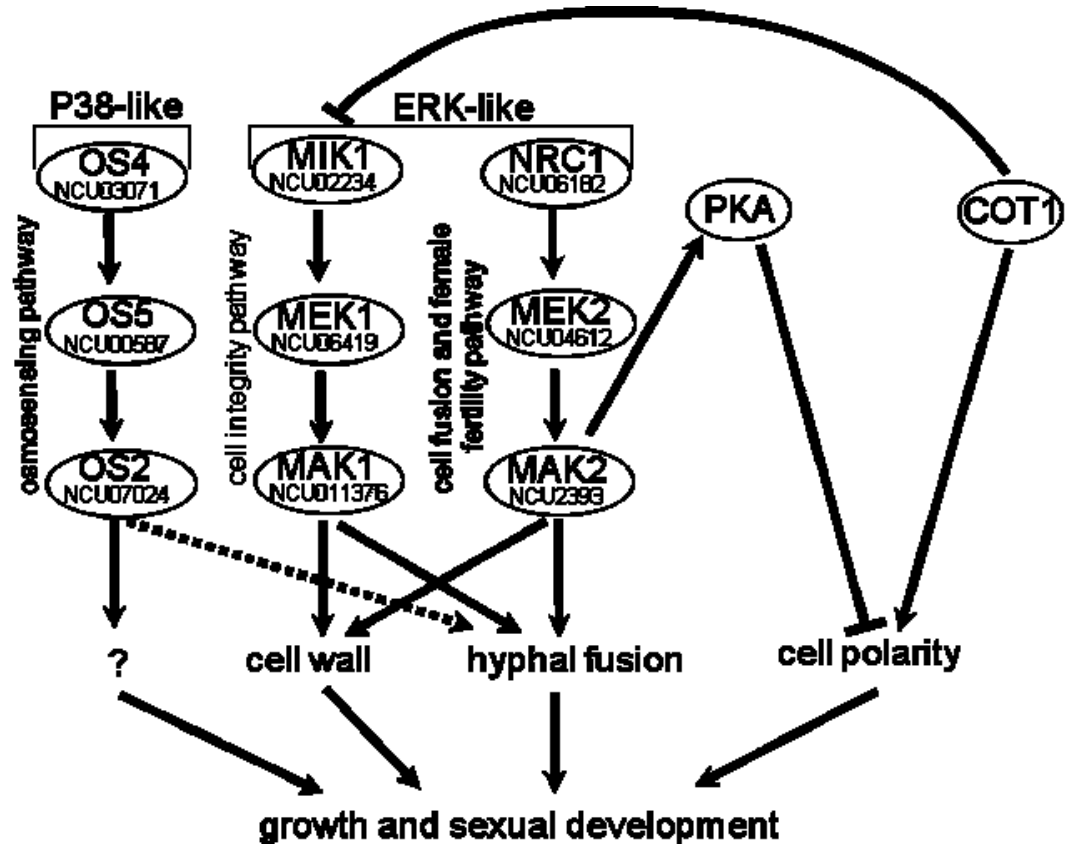

Figure II-7 Model summarizing the components and functions of the three $\mathbf{N}$. crassa MAPK modules and cross-communication between COT1, MAP kinase and PKA signaling pathways. Details are discussed in the text.

We found it interesting that all mutants described here and in the literature that lack female reproductive structures are also cell-fusion defective. This is best documented in mutants characterized in N. crassa (Perkins et al., 2001, Fleissner et al., 2005, Xiang et al., 2002, Wilson \& Dempsey, 1999) and the closely related fungus Sordaria macrospora (Poggeler \& Kuck, 2004, Engh et al., 2007), but was also observed in A. nidulans (Wei et al., 2003). The hypothesis that hyphal fusion is functionally linked with sexual fruiting body formation is also supported by our characterization of the suppression of the mak-2 pathway by cot-1(ts): the lack or delay of hyphal fusion correlated with defects in the formation of protoperithecia. Furthermore, hyphal fusion has been shown to occur in the fruiting bodies of basidiomycete species (Williams, 1985). However, it is currently still 
unclear whether hyphal fusion is a prerequisite for the formation of female reproductive structures (Glass et al., 2004, Poggeler et al., 2006).

Our genetic analysis suggests that the MAK1, MAK2 and COT1 signaling pathways in $N$. crassa are linked (Figure II-7). This is best characterized by the gulliver-type suppression of the cot-1(ts) growth defects at restrictive conditions observed in mutants that harbor mak-2 pathway deletions. We have recently presented evidence indicating that inhibiting PKA activity can suppress the cot-1(ts) phenotype (Gorovits \& Yarden, 2003, Seiler et al., 2006). Here, we demonstrate that the loss of MAK2 activity can also partially suppress the cot-1(ts) phenotype. It is tempting to speculate that the observed reduction in PKA activity in $\Delta$ mak-2 may be involved in the suppression mechanism, thus establishing a potential MAK2-PKA interaction in N. crassa. Extensive literature supports the occurrence of direct cross-talk between PKA and MAPK signaling in various organisms (Lengeler et al., 2000, Pan et al., 2000, Stork \& Schmitt, 2002, Mosch et al., 1999). However, cross-talk between these two pathways is generally directed from PKA towards the MAPK pathway and not vice versa. One of the few examples of MAPK to PKA signaling is the phosphorylation of the phospho-diesterase RegA by Erk2 in Dictyostelium discoideum that results in the degradation of the CAMP-specific diesterase and thereby the activation of PKA (Loomis, 1998, Mohanty et al., 2001). Alternatively, a common upstream link between MAPK and PKA (e.g. via the small GTPase RAS) may be responsible for coordinating the activity intensities of the MAPK and PKA pathways in a manner that confers the observed phenotypes. If this is the case, additional gulliver-type suppressors may serve as a tool to further define the MAK2/PKA pathways in N. crassa.

Another example of the link between COT1 and MAPK signaling is the suppression of mak-2 pathway defects by cot-1(ts). A candidate component of this link is MAK1, whose activity was increased in cot-1(ts). Based on the phenotypic similarities of mak-1 and mak2 pathway deletions, we suggest that both pathways have partially overlapping functions and that the increase in phospho-MAK1 in cot-1(ts) can compensate, at least in part, for the loss of mak-2 pathway functions. An interesting open question is why is this compensation mechanism specific for $\Delta m a k-2$ ? One possible explanation may be that the primary interaction between COT1 and MAPKs is via MAK1. This is supported by studies in yeasts and animals indicating the presence of a link between $\mathrm{Ndr}$ kinases and Rho-type GTPase. Genetic data in $S$. cerevisiae suggest that the COT1 homolog Cbk1p may 
negatively regulate the small GTPase Rho1p, which in turn activates the cell wall integrity pathway that is most similar to the N. crassa MAK1 pathway (Jorgensen et al., 2002, Schneper et al., 2004, Versele \& Thevelein, 2001). A physical interaction has also been shown to exist between the Ndr kinase ORB6 and the Rho-GTPase activating protein RGA4 in fission yeast (Das et al., 2007). An indication that this connection may be conserved between fungi and animals has been provided by studies in Drosophila melanogaster and Caenorhabditis elegans, which also describe genetic interactions between Ndr kinases and RhoA (Zallen et al., 2000, Emoto et al., 2004). Thus, the connection between COT1, MAK1 and MAK2 signaling during hyphal growth may provide insights in the regulation of morphogenesis in other highly polar cells such as neurons or pollen tubes. 


\section{Material and Methods}

\section{Strains, media and growth conditions}

General genetic procedures and media used in the handling of $N$. crassa have been described (Davis \& DeSerres, 1970) or are available through the Fungal Genetic Stock Center (www.fgsc.net), with the exception of genetic crosses, which were performed on $2 \%$ cornmeal agar (Sigma, USA) supplemented with $0.1 \%$ glucose. This complex, lownitrogen containing media increased the success rate of crosses with strains that are difficult/impossible to cross on standard synthetic crossing media such as gul-4 and most MAPK mutants, and the hyg ${ }^{\mathrm{R}}$ and cot-1 marker segregate perfectly in crosses which produce viable spores. Also, the terminal phenotype of mutants defective in sexual reproduction could be determined in a more reliable manner on this media compared to synthetic crossing media (Muller et al, 1995). Strains were grown in either liquid or solid (supplemented with $1.5 \%$ agar) Vogel's minimal media with $2 \%(\mathrm{w} / \mathrm{v})$ sucrose, unless otherwise stated. When required, $5 \mu \mathrm{M}$ KT5720, $500 \mu \mathrm{M} \mathrm{Br}$-cAMP or $5 \mathrm{mg} / \mathrm{ml}$ lysing enzymes, all purchased from Sigma, were added. Gradient plates contained solid Vogel's minimal media with $1 \%$ sucrose $(\mathrm{w} / \mathrm{v})$ and $1 \%$ sorbose $(\mathrm{w} / \mathrm{v})$ to restrict the radial growth rate. Inhibitors were added at $50^{\circ} \mathrm{C}$, the plates slanted during the solidification of the agar, then overlaid with an equal volume of the same medium lacking additives in horizontal position and incubated for 1 day to allow equal diffusion of the additive. To induce stress-dependent MAPK signaling, $\mathrm{H}_{2} \mathrm{O}_{2}(7 \mathrm{mM})$ or $\mathrm{NaCl}(1 \mathrm{M})$ were added to liquid cultures of the relevant strains two hours prior to harvesting. Stress induction by temperature shift was achieved by germinating the strains for $15 \mathrm{~h}$ on cellophane covered agar plates, followed by a shift to $37^{\circ} \mathrm{C}$ for $10 \mathrm{~h}$. For protein extraction, the mycelial sheet was peeled off the cellophane and plunged into liquid nitrogen.

The gul-4/mak-2 complementation construct was generated by amplifying the mak-2 ORF using the primers 2393-Not-5' (ATC GGC GGC CGC CAT GAG CAG CGC ACA AAG AGG CG) and 52393-Not-3' (ATC GGC GGC CGC TCA CCT CAT AAT CTC CTG GTA GAT C) designed to introduce Notl restriction sites. The Notl-digested PCR product was cloned into the expression vector pEHN1nat (kindly provided by Stephanie Poeggler), which allowed the 
expression of mak-2 via $A$. nidulans gpd promotor and trpC terminator sequences. DNAmediated transformation of $N$. crassa protoplasts was carried out as described (Vollmer \& Yanofsky, 1986). The nourseothricin concentration was adjusted to $30 \mu \mathrm{g} / \mathrm{ml}$ to select for transformants.

Strains used in this study are listed in Table II-2 (see also (McCluskey, 2003). gul-4 was mapped by introducing the auxotrophic markers $\arg -10$, $\arg -11$ and met-7 into the cot1(ts) background and subsequently crossing the obtained double mutants with gul-4;cot1 (ts). Progeny were plated on Vogels minimal media containing $0.005 \%$ sucrose and $2 \%$ sorbose at $25^{\circ} \mathrm{C}$, overlaid with Vogels minimal media containing $2 \%$ sucrose after 2 days and incubated for additional 5-10 days at $37^{\circ} \mathrm{C}$. The ratio between cot-1 and cot-1;gul-4 progeny was scored by stereomicroscopy and indicated the linkage of gul-4 with the auxotrophic marker.

Table II-2 Neurospora crassa strains used in this study.

\begin{tabular}{|c|c|c|}
\hline Strain & Genotype & Source \\
\hline wild type & 74-OR23-1A & FGSC \#987 \\
\hline $\cot -1$ (ts) & $\cot -1(C 102 t)$ & FGSC \#4066 \\
\hline gul-4;pe;fl;cot-1;inl & gul-4 pe fl cot-1 inl & FGSC \#1173 \\
\hline $\cot -1 ; g u l-4$ & gul-4 cot-1 & This study \\
\hline$\Delta o s-4$ (heterokaryon) & hph::os-4 4 bar::mus-51 + bar::mus-51 & FGSC \#11479 \\
\hline$\Delta o s-4$ (microconidia) & hph::os-4A bar::mus-51 & This study \\
\hline$\Delta n r c-1$ (heterokaryon) & hph::nrc-1A bar::mus-51 + bar::mus-51 & FGSC \#11466 \\
\hline$\Delta n r c-1$ & hph::nrc-1 & This study \\
\hline$\Delta m i k-1$ & hph::mik-1A & FGSC \#11326 \\
\hline$\Delta o s-5$ (heterokaryon) & hph::os-54 bar::mus-51 + bar::mus-51 & FGSC \#11480 \\
\hline$\Delta o s-5$ & hph::os-5 & This study \\
\hline$\Delta m e k-2$ (heterokaryon) & hph::mek-24 bar::mus-51 + bar::mus-51 & FGSC \#11481 \\
\hline$\Delta m e k-2$ (microconidia) & hph::mek-2A bar::mus-51 & This study \\
\hline$\Delta m e k-1$ & hph::mek-1A & FGSC \#11318 \\
\hline$\Delta o s-2$ & hph::os-2A & FGSC \#11436 \\
\hline$\Delta m a k-2$ & hph::mak-2A & Li et al., 2005 \\
\hline$\Delta m a k-1$ & hph::mak-1A & FGSC \#11321 \\
\hline$\Delta n r c-1 ; \cot -1(t s)$ & $h p h:: n r c-1$ cot-1(C102t) & This study \\
\hline$\Delta o s-5 ; \cot -1$ (ts) & hph::os-5 $\Delta$ cot-1(C102t) & This study \\
\hline$\Delta m e k-1 ; \cot -1(t s)$ & hph::mek-1A cot-1(C102t) & This study \\
\hline$\Delta o s-2 ; \cot -1(t s)$ & hph::os-2A cot-1(C102t) & This study \\
\hline$\Delta m a k-2 ; \cot -1$ (ts) & hph::mak-2A cot-1(C102t) & This study \\
\hline$\Delta m a k-1 ; \cot -1$ (ts) & hph::mak-1A cot-1(C102t) & This study \\
\hline$m c b(14-4)$ & $m c b(14-4)$ & Seiler and Plamann, 2003 \\
\hline$\Delta m a k-2 ; m c b(14-4)$ & hph::mak-1А mcb(14-4) & This study \\
\hline
\end{tabular}




\section{Protein extraction, immunoblotting and PKA activity measurement}

Western blot analysis was performed as previously described (Gorovits \& Yarden, 2003). Briefly, N. crassa mycelial samples were frozen in liquid nitrogen, pulverized, and suspended in lysis buffer [1 M sorbitol, 10 mM HEPES (pH 7.5), 5 mM EDTA, 5 mM EGTA, $5 \mathrm{mM} \mathrm{NaF}, 0.1 \mathrm{M} \mathrm{KCl}, 0.2 \%$ Triton X-100 and Complete ${ }^{\mathrm{TM}}$ (Roche Applied Science, Germany) protease inhibitor mixture]. The samples were homogenized by 10 strokes of pestle $A$ in a Dounce homogenizer. The homogenates were centrifuged for $40 \mathrm{~min}$ at $10,000 \mathrm{~g}$ and the supernatant recovered and stored at $-70^{\circ} \mathrm{C}$ until analysis. Proteins were separated by 7.5 or $10 \%$ SDS-PAGE and subsequently blotted onto nitrocellulose membranes. Antibodies used throughout this study included anti-COT1 (Gorovits et al., 1999), anti-PhosphoMAPK (Cell Signaling Technology, USA), monoclonal $9 E 10$ anti-cMYC (Santa Cruz, USA) and goat peroxidase-coupled secondary antibody (Amersham Biosciences, Germany).

PKA assays were performed as previously described (Ziv et al., 2007) with minor modifications. Specifically, $10^{6}$ conidia/ml were shaken for $11 \mathrm{~h}$ in pre-warmed $\left(36^{\circ} \mathrm{C}\right)$ Vogel's sucrose minimal medium. The cultures were harvested by centrifugation (10 min, $3.000 \mathrm{~g}, 4^{\circ} \mathrm{C}$ ) and immediately assayed for PKA activity. Differences in kemptide phosphorylation were determined by densitometry and subjected to paired two sample tTest analyses.

\section{Microscopy}

Samples were viewed with an ORCA ER digital camera (Hamamatsu, Japan) mounted on an Axiovert S100 microscope (Zeiss, Germany). Image acquisition was done using the Openlab 5.01 software (Improvision, United Kingdom) and images were further processed using Photoshop CS2 (Adobe, USA). Low magnification documentation of fungal hyphae or colonies was performed with an SZX12 stereomicroscope (Olympus, Japan) and a PS30 camera (Kappa, Germany). 


\section{Acknowledgments}

This research project was financially supported by the German Bundesland of Lower Saxony and the Volkswagen Foundation (SS and OY), by the Deutsche Forschungsgemeinschaft through the DFG Research Center of Molecular Physiology of the Brain (CMPB) and the DFG priority program "Cell Polarity" (SS), and by The Israel Science Foundation (OY). We thank Stephanie Poeggeler for kindly providing us with plasmid pEHN1nat. 


\section{Supplementary Data}
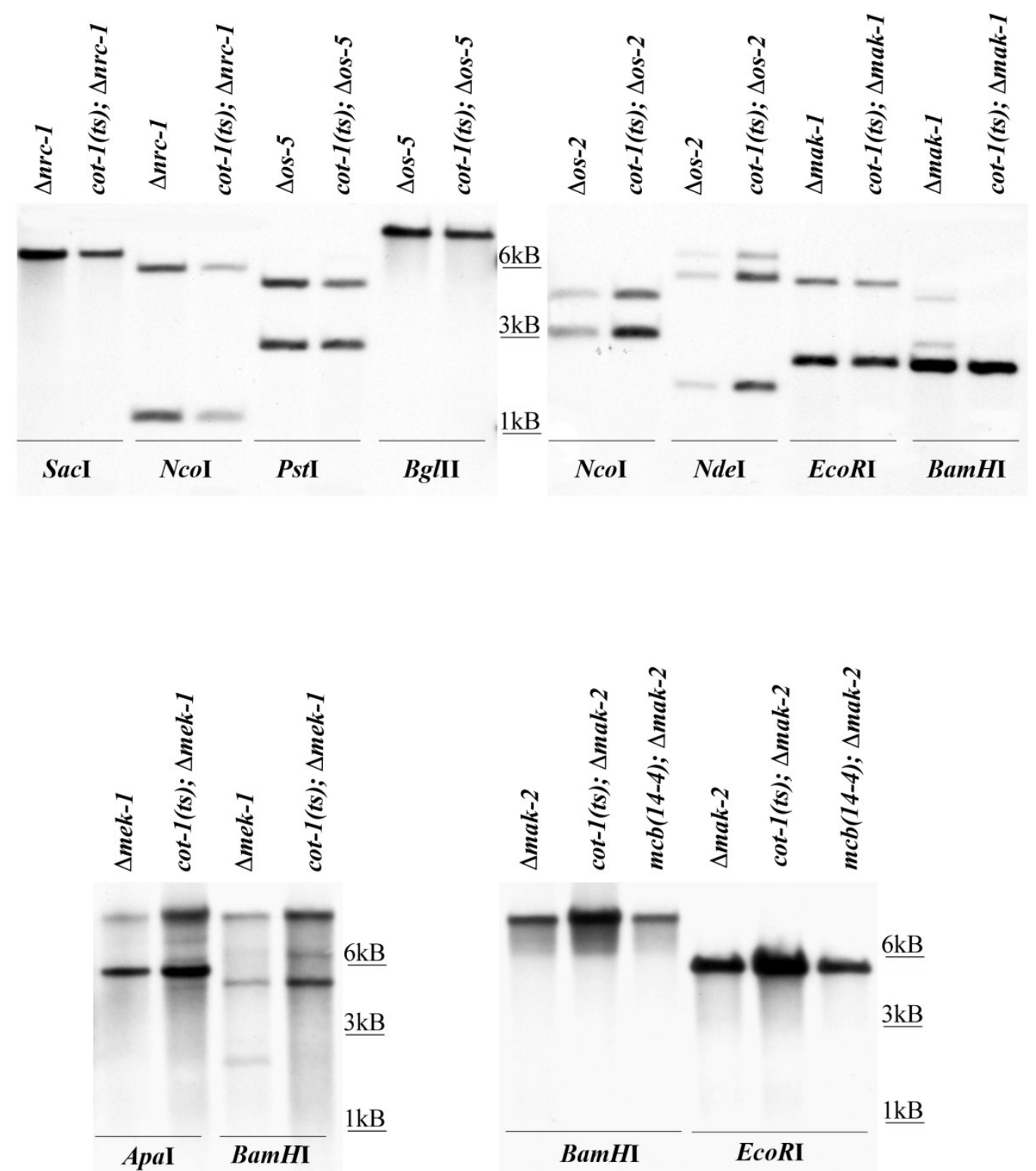

Figure S II-1 Southern blot analysis of the mutant strains generated throughout this work. The hyg ${ }^{\mathrm{R}}$ resistance cassette was used to probe genomic DNA of the cot-1;mak double mutants and respective mak single mutants digested with the indicated restriction enzymes. The identical restriction patterns of the mapk single and cot-1;mapk double mutants confirmed the mapk deletion genotypes in the corresponding strains. 


\section{References}

Bahn, Y. S., Xue, C., Idnurm, A., Rutherford, J. C., Heitman, J. \& Cardenas, M. E., (2007) Sensing the environment: lessons from fungi. Nat Rev Microbiol 5: 57-69.

Bistis, G. N., Perkins, D. D. \& Read, N. D., (2003) Different cell types in Neurospora crassa. Fungal Genet. Newsl. 50: 17-19.

Borkovich, K. A., Alex, L. A., Yarden, O., Freitag, M., Turner, G. E., Read, N. D., Seiler, S., Bell-Pedersen, D., Paietta, J., Plesofsky, N., Plamann, M., Goodrich-Tanrikulu, M., Schulte, U., Mannhaupt, G., Nargang, F. E., Radford, A., Selitrennikoff, C., Galagan, J. E., Dunlap, J. C., Loros, J. J., Catcheside, D., Inoue, H., Aramayo, R., Polymenis, M., Selker, E. U., Sachs, M. S., Marzluf, G. A., Paulsen, I., Davis, R., Ebbole, D. J., Zelter, A., Kalkman, E. R., O'Rourke, R., Bowring, F., Yeadon, J., Ishii, C., Suzuki, K., Sakai, W. \& Pratt, R., (2004) Lessons from the genome sequence of Neurospora crassa: tracing the path from genomic blueprint to multicellular organism. Microbiol Mol Biol Rev 68: 1-108.

Bruno, K. S., Aramayo, R., Minke, P. F., Metzenberg, R. L. \& Plamann, M., (1996a) Loss of growth polarity and mislocalization of septa in a Neurospora mutant altered in the regulatory subunit of cAMP-dependent protein kinase. Embo J 15: 5772-5782.

Bruno, K. S., Tinsley, J. H., Minke, P. F. \& Plamann, M., (1996b) Genetic interactions among cytoplasmic dynein, dynactin, and nuclear distribution mutants of Neurospora crassa. Proc Natl Acad Sci U S A 93: 4775-4780.

Bussink, H. J. \& Osmani, S. A., (1999) A mitogen-activated protein kinase (MPKA) is involved in polarized growth in the filamentous fungus, Aspergillus nidulans. FEMS Microbiol Lett 173: 117-125.

D'Souza, C. A. \& Heitman, J., (2001) Conserved cAMP signaling cascades regulate fungal development and virulence. FEMS Microbiol Rev 25: 349-364.

Dan, I., Watanabe, N. M. \& Kusumi, A., (2001) The Ste20 group kinases as regulators of MAP kinase cascades. Trends Cell Biol 11: 220-230.

Das, M., Wiley, D. J., Medina, S., Vincent, H. A., Larrea, M., Oriolo, A. \& Verde, F., (2007) Regulation of cell diameter, For3p localization, and cell symmetry by fission yeast Rho-GAP Rga4p. Mol Biol Cell 18: 2090-2101.

Davis, R. D. \& DeSerres, F. J., (1970) Genetic and microbiological research techniques for Neurospora crassa. Methods Enzymol. 17: 79-143.

Dean, R. A., Talbot, N. J., Ebbole, D. J., Farman, M. L., Mitchell, T. K., Orbach, M. J., Thon, M., Kulkarni, R., Xu, J. R., Pan, H., Read, N. D., Lee, Y. H., Carbone, I., Brown, D., Oh, Y. Y., Donofrio, N., Jeong, J. S., Soanes, D. M., Djonovic, S., Kolomiets, E., Rehmeyer, C., Li, W., Harding, M., Kim, S., Lebrun, M. H., Bohnert, H., Coughlan, S., Butler, J., Calvo, S., Ma, L. J., Nicol, R., Purcell, S., Nusbaum, C., Galagan, J. E. \& Birren, B. W., (2005) The genome sequence of the rice blast fungus Magnaporthe grisea. Nature 434: 980-986.

Dunlap, J. C., Borkovich, K. A., Henn, M. R., Turner, G. E., Sachs, M. S., Glass, N. L., McCluskey, K., Plamann, M., Galagan, J. E., Birren, B. W., Weiss, R. L., Townsend, J. P., Loros, J. J., Nelson, M. A., Lambreghts, R., Colot, H. V., Park, G., Collopy, P., 
Ringelberg, C., Crew, C., Litvinkova, L., DeCaprio, D., Hood, H. M., Curilla, S., Shi, M., Crawford, M., Koerhsen, M., Montgomery, P., Larson, L., Pearson, M., Kasuga, T., Tian, C., Basturkmen, M., Altamirano, L. \& Xu, J., (2007) Enabling a community to dissect an organism: overview of the Neurospora functional genomics project. Adv Genet 57: 49-96.

Emoto, K., He, Y., Ye, B., Grueber, W. B., Adler, P. N., Jan, L. Y. \& Jan, Y. N., (2004) Control of dendritic branching and tiling by the Tricornered-kinase/Furry signaling pathway in Drosophila sensory neurons. Cell 119: 245-256.

Emoto, K., Parrish, J. Z., Jan, L. Y. \& Jan, Y. N., (2006) The tumour suppressor Hippo acts with the NDR kinases in dendritic tiling and maintenance. Nature 443: 210-213.

Engh, I., Wurtz, C., Witzel-Schlomp, K., Zhang, H. Y., Hoff, B., Nowrousian, M., Rottensteiner, H. \& Kuck, U., (2007) The WW domain protein PRO40 is required for fungal fertility and associates with Woronin bodies. Eukaryot Cell 6: 831-843.

Fleissner, A., Sarkar, S., Jacobson, D. J., Roca, M. G., Read, N. D. \& Glass, N. L., (2005) The so locus is required for vegetative cell fusion and postfertilization events in Neurospora crassa. Eukaryot Cell 4: 920-930.

Fujimura, M., Ochiai, N., Oshima, M., Motoyama, T., Ichiishi, A., Usami, R., Horikoshi, K. \& Yamaguchi, I., (2003) Putative homologs of SSK22 MAPKK kinase and PBS2 MAPK kinase of Saccharomyces cerevisiae encoded by os-4 and os-5 genes for osmotic sensitivity and fungicide resistance in Neurospora crassa. Biosci Biotechnol Biochem 67: 186-191.

Furukawa, K., Hoshi, Y., Maeda, T., Nakajima, T. \& Abe, K., (2005) Aspergillus nidulans HOG pathway is activated only by two-component signalling pathway in response to osmotic stress. Mol Microbiol 56: 1246-1261.

Galagan, J. E., Calvo, S. E., Borkovich, K. A., Selker, E. U., Read, N. D., Jaffe, D., FitzHugh, W., Ma, L. J., Smirnov, S., Purcell, S., Rehman, B., Elkins, T., Engels, R., Wang, S., Nielsen, C. B., Butler, J., Endrizzi, M., Qui, D., lanakiev, P., Bell-Pedersen, D., Nelson, M. A., Werner-Washburne, M., Selitrennikoff, C. P., Kinsey, J. A., Braun, E. L., Zelter, A., Schulte, U., Kothe, G. O., Jedd, G., Mewes, W., Staben, C., Marcotte, E., Greenberg, D., Roy, A., Foley, K., Naylor, J., Stange-Thomann, N., Barrett, R., Gnerre, S., Kamal, M., Kamvysselis, M., Mauceli, E., Bielke, C., Rudd, S., Frishman, D., Krystofova, S., Rasmussen, C., Metzenberg, R. L., Perkins, D. D., Kroken, S., Cogoni, C., Macino, G., Catcheside, D., Li, W., Pratt, R. J., Osmani, S. A., DeSouza, C. P., Glass, L., Orbach, M. J., Berglund, J. A., Voelker, R., Yarden, O., Plamann, M., Seiler, S., Dunlap, J., Radford, A., Aramayo, R., Natvig, D. O., Alex, L. A., Mannhaupt, G., Ebbole, D. J., Freitag, M., Paulsen, I., Sachs, M. S., Lander, E. S., Nusbaum, C. \& Birren, B., (2003) The genome sequence of the filamentous fungus Neurospora crassa. Nature 422: 859-868.

Galagan, J. E., Calvo, S. E., Cuomo, C., Ma, L. J., Wortman, J. R., Batzoglou, S., Lee, S. I., Basturkmen, M., Spevak, C. C., Clutterbuck, J., Kapitonov, V., Jurka, J., Scazzocchio, C., Farman, M., Butler, J., Purcell, S., Harris, S., Braus, G. H., Draht, O., Busch, S., D'Enfert, C., Bouchier, C., Goldman, G. H., Bell-Pedersen, D., Griffiths-Jones, S., Doonan, J. H., Yu, J., Vienken, K., Pain, A., Freitag, M., Selker, E. U., Archer, D. B., Penalva, M. A., Oakley, B. R., Momany, M., Tanaka, T., Kumagai, T., Asai, K., Machida, M., Nierman, W. C., Denning, D. W., Caddick, M., Hynes, M., Paoletti, M., 
Fischer, R., Miller, B., Dyer, P., Sachs, M. S., Osmani, S. A. \& Birren, B. W., (2005) Sequencing of Aspergillus nidulans and comparative analysis with A. fumigatus and A. oryzae. Nature 438: 1105-1115.

Geng, W., He, B., Wang, M. \& Adler, P. N., (2000) The tricornered gene, which is required for the integrity of epidermal cell extensions, encodes the Drosophila nuclear DBF2-related kinase. Genetics 156: 1817-1828.

Glass, N. L., Rasmussen, C., Roca, M. G. \& Read, N. D., (2004) Hyphal homing, fusion and mycelial interconnectedness. Trends Microbio/ 12: 135-141.

Gorovits, R., Propheta, O., Kolot, M., Dombradi, V. \& Yarden, O., (1999) A mutation within the catalytic domain of COT1 kinase confers changes in the presence of two COT1 isoforms and in Ser/Thr protein kinase and phosphatase activities in Neurospora crassa. Fungal Genet Biol 27: 264-274.

Gorovits, R. \& Yarden, O., (2003) Environmental suppression of Neurospora crassa cot-1 hyperbranching: a link between COT1 kinase and stress sensing. Eukaryot Cell 2: 699-707.

Hampsey, M., (1997) A review of phenotypes in Saccharomyces cerevisiae. Yeast 13: 1099-1133.

Harris, S. D., (2006) Cell polarity in filamentous fungi: shaping the mold. Int Rev Cyto/ 251: 41-77.

Hergovich, A., Stegert, M. R., Schmitz, D. \& Hemmings, B. A., (2006) NDR kinases regulate essential cell processes from yeast to humans. Nat Rev Mol Cell Biol 7: 253-264.

Hou, Z., Xue, C., Peng, Y., Katan, T., Kistler, H. C. \& Xu, J. R., (2002) A mitogen-activated protein kinase gene (MGV1) in Fusarium graminearum is required for female fertility, heterokaryon formation, and plant infection. Mol Plant Microbe Interact 15: 1119-1127.

Jones, C. A., Greer-Phillips, S. E. \& Borkovich, K. A., (2007) The Response Regulator RRG-1 Functions Upstream of a Mitogen-activated Protein Kinase Pathway Impacting Asexual Development, Female Fertility, Osmotic Stress, and Fungicide Resistance in Neurospora crassa. Mol. Biol. Cell 18: 2123-2136.

Jorgensen, P., Nelson, B., Robinson, M. D., Chen, Y., Andrews, B., Tyers, M. \& Boone, C., (2002) High-resolution genetic mapping with ordered arrays of Saccharomyces cerevisiae deletion mutants. Genetics 162: 1091-1099.

Kanai, M., Kume, K., Miyahara, K., Sakai, K., Nakamura, K., Leonhard, K., Wiley, D. J., Verde, F., Toda, T. \& Hirata, D., (2005) Fission yeast MO25 protein is localized at SPB and septum and is essential for cell morphogenesis. Embo J 24: 3012-3025.

Kawasaki, L., Sanchez, O., Shiozaki, K. \& Aguirre, J., (2002) SakA MAP kinase is involved in stress signal transduction, sexual development and spore viability in Aspergillus nidulans. Mol Microbiol 45: 1153-1163.

Kojima, K., Kikuchi, T., Takano, Y., Oshiro, E. \& Okuno, T., (2002) The mitogen-activated protein kinase gene MAF1 is essential for the early differentiation phase of appressorium formation in Colletotrichum lagenarium. Mol Plant Microbe Interact 15: $1268-1276$. 
Kothe, G. O. \& Free, S. J., (1998) The isolation and characterization of nrc-1 and nrc-2, two genes encoding protein kinases that control growth and development in Neurospora crassa. Genetics 149: 117-130.

Lengeler, K. B., Davidson, R. C., D'Souza, C., Harashima, T., Shen, W. C., Wang, P., Pan, X., Waugh, M. \& Heitman, J., (2000) Signal transduction cascades regulating fungal development and virulence. Microbiol Mol Biol Rev 64: 746-785.

Lewis, T. S., Shapiro, P. S. \& Ahn, N. G., (1998) Signal transduction through MAP kinase cascades. Adv Cancer Res 74: 49-139.

Li, D., Bobrowicz, P., Wilkinson, H. H. \& Ebbole, D. J., (2005) A mitogen-activated protein kinase pathway essential for mating and contributing to vegetative growth in Neurospora crassa. Genetics 170: 1091-1104.

Loomis, W. F., (1998) Role of PKA in the timing of developmental events in Dictyostelium cells. Microbiol Mol Biol Rev 62: 684-694.

Madhani, H. D. \& Fink, G. R., (1998) The riddle of MAP kinase signaling specificity. Trends Genet 14: 151-155.

Maeda, T., Takekawa, M. \& Saito, H., (1995) Activation of yeast PBS2 MAPKK by MAPKKKs or by binding of an SH3-containing osmosensor. Science 269: 554-558.

McCluskey, K., (2003) The Fungal Genetics Stock Center: from molds to molecules. Adv Appl Microbiol 52: 245-262.

Mey, G., Held, K., Scheffer, J., Tenberge, K. B. \& Tudzynski, P., (2002) CPMK2, an SLT2homologous mitogen-activated protein (MAP) kinase, is essential for pathogenesis of Claviceps purpurea on rye: evidence for a second conserved pathogenesisrelated MAP kinase cascade in phytopathogenic fungi. Mol Microbiol 46: 305-318.

Mohanty, S., Lee, S., Yadava, N., Dealy, M. J., Johnson, R. S. \& Firtel, R. A., (2001) Regulated protein degradation controls PKA function and cell-type differentiation in Dictyostelium. Genes Dev 15: 1435-1448.

Monge, R. A., Roman, E., Nombela, C. \& Pla, J., (2006) The MAP kinase signal transduction network in Candida albicans. Microbiology 152: 905-912.

Mosch, H. U., Kubler, E., Krappmann, S., Fink, G. R. \& Braus, G. H., (1999) Crosstalk between the Ras2p-controlled mitogen-activated protein kinase and CAMP pathways during invasive growth of Saccharomyces cerevisiae. Mol Biol Cell 10: 1325-1335.

Nelson, B., Kurischko, C., Horecka, J., Mody, M., Nair, P., Pratt, L., Zougman, A., McBroom, L. D., Hughes, T. R., Boone, C. \& Luca, F. C., (2003) RAM: a conserved signaling network that regulates Ace $2 p$ transcriptional activity and polarized morphogenesis. Mol Biol Cell 14: 3782-3803.

Noguchi, R., Banno, S., Ichikawa, R., Fukumori, F., Ichiishi, A., Kimura, M., Yamaguchi, I. \& Fujimura, M., (2007) Identification of OS-2 MAP kinase-dependent genes induced in response to osmotic stress, antifungal agent fludioxonil, and heat shock in Neurospora crassa. Fungal Genet Biol 44: 208-218.

Palanivelu, R. \& Preuss, D., (2000) Pollen tube targeting and axon guidance: parallels in tip growth mechanisms. Trends Cell Biol 10: 517-524. 
Pan, X., Harashima, T. \& Heitman, J., (2000) Signal transduction cascades regulating pseudohyphal differentiation of Saccharomyces cerevisiae. Curr Opin Microbiol 3: 567-572.

Pandey, A., Roca, M. G., Read, N. D. \& Glass, N. L., (2004) Role of a mitogen-activated protein kinase pathway during conidial germination and hyphal fusion in Neurospora crassa. Eukaryot Cell 3: 348-358.

Perkins, D. D., Radford, A. \& Sachs, M. S., (2001) The Neurospora compendium. Academic Press.

Poggeler, S. \& Kuck, U., (2004) A WD40 repeat protein regulates fungal cell differentiation and can be replaced functionally by the mammalian homologue striatin. Eukaryot Cell 3: 232-240.

Poggeler, S., Nowrousian, M. \& Kuck, U., (2006) Fruiting-Body Development in Ascomycetes. In: The Mycota I. U. Kües \& R. Fischer (eds). Heidelberg: Springer, pp. 325-355.

Posas, F. \& Saito, H., (1998) Activation of the yeast SSK2 MAP kinase kinase kinase by the SSK1 two-component response regulator. Embo J 17: 1385-1394.

Qi, M. \& Elion, E. A., (2005) MAP kinase pathways. J Cell Sci 118: 3569-3572.

Racki, W. J., Becam, A. M., Nasr, F. \& Herbert, C. J., (2000) Cbk1p, a protein similar to the human myotonic dystrophy kinase, is essential for normal morphogenesis in Saccharomyces cerevisiae. Embo J 19: 4524-4532.

Schneper, L., Krauss, A., Miyamoto, R., Fang, S. \& Broach, J. R., (2004) The Ras/protein kinase A pathway acts in parallel with the Mob2/Cbk1 pathway to effect cell cycle progression and proper bud site selection. Eukaryot Cell 3: 108-120.

Seiler, S., Vogt, N., Ziv, C., Gorovits, R. \& Yarden, O., (2006) The STE20/germinal center kinase POD6 interacts with the NDR kinase COT1 and is involved in polar tip extension in Neurospora crassa. Mol Biol Cell 17: 4080-4092.

Stegert, M. R., Hergovich, A., Tamaskovic, R., Bichsel, S. J. \& Hemmings, B. A., (2005) Regulation of NDR protein kinase by hydrophobic motif phosphorylation mediated by the mammalian Ste20-like kinase MST3. Mol Cell Biol 25: 11019-11029.

Stork, P. J. \& Schmitt, J. M., (2002) Crosstalk between CAMP and MAP kinase signaling in the regulation of cell proliferation. Trends Cell Biol 12: 258-266.

Terenzi, H. F. \& Reissig, J. L., (1967) Modifiers of the cot gene in Neurospora: the gulliver mutants. Genetics 56: 321-329.

Verde, F., Wiley, D. J. \& Nurse, P., (1998) Fission yeast orb6, a ser/thr protein kinase related to mammalian rho kinase and myotonic dystrophy kinase, is required for maintenance of cell polarity and coordinates cell morphogenesis with the cell cycle. Proc Natl Acad Sci U S A 95: 7526-7531.

Versele, M. \& Thevelein, J. M., (2001) Lre1 affects chitinase expression, trehalose accumulation and heat resistance through inhibition of the Cbk1 protein kinase in Saccharomyces cerevisiae. Mol Microbiol 41: 1311-1326.

Vollmer, S. J. \& Yanofsky, C., (1986) Efficient cloning of genes of Neurospora crassa. Proc Natl Acad Sci U S A 83: 4869-4873. 
Wei, H., Requena, N. \& Fischer, R., (2003) The MAPKK kinase SteC regulates conidiophore morphology and is essential for heterokaryon formation and sexual development in the homothallic fungus Aspergillus nidulans. Mol Microbiol 47: 1577-1588.

Williams, M. A. J., (1985) Ultrastructural aspects of fruit body differentiation in Flammulina velutipes. In: Developmental Biology of Higher Fungi. D. Moore (ed). Cambridge: University Press, pp. 429-450.

Wilson, J. F. \& Dempsey, J. A., (1999) A hyphal fusion mutant in Neurospora crassa. Fungal Genetics Newsletter 46: 31.

Xiang, Q., Rasmussen, C. \& Glass, N. L., (2002) The ham-2 locus, encoding a putative transmembrane protein, is required for hyphal fusion in Neurospora crassa. Genetics 160: 169-180.

Xu, J. R., (2000) Map kinases in fungal pathogens. Fungal Genet Biol 31: 137-152.

Xu, J. R., Staiger, C. J. \& Hamer, J. E., (1998) Inactivation of the mitogen-activated protein kinase Mps1 from the rice blast fungus prevents penetration of host cells but allows activation of plant defense responses. Proc Natl Acad Sci U S A 95: 1271312718.

Xu, J. R., Zhao, X. \& Dean, R. A., (2007) From genes to genomes: a new paradigm for studying fungal pathogenesis in Magnaporthe oryzae. Adv Genet 57: 175-218.

Yarden, O., Plamann, M., Ebbole, D. J. \& Yanofsky, C., (1992) cot-1, a gene required for hyphal elongation in Neurospora crassa, encodes a protein kinase. Embo J 11: 2159-2166.

Zallen, J. A., Peckol, E. L., Tobin, D. M. \& Bargmann, C. I., (2000) Neuronal cell shape and neurite initiation are regulated by the $\mathrm{Ndr}$ kinase SAX-1, a member of the Orb6/COT-1/warts serine/threonine kinase family. Mol Biol Cell 11: 3177-3190.

Zhang, Y., Lamm, R., Pillonel, C., Lam, S. \& Xu, J. R., (2002) Osmoregulation and fungicide resistance: the Neurospora crassa os-2 gene encodes a HOG1 mitogen-activated protein kinase homologue. Appl Environ Microbiol 68: 532-538.

Ziv, C., Gorovits, R. \& Yarden, O., (2008) Carbon source affects PKA-dependent polarity of Neurospora crassa in a CRE-1-dependent and independent manner. Fungal Genet Biol 45: 103-116. 


\title{
CHAPTER III
}

\section{Two NDR kinase - MOB complexes function as distinct modules during septum formation and tip extension in Neurospora crassa}

\begin{abstract}
NDR kinases are important for growth and differentiation and require interaction with MOB proteins for activity and function. We characterized the NDR kinases and MOB activators in Neurospora crassa and identified two NDR kinases (COT1 and DBF2) and four MOB proteins (MOB1, MOB2A, MOB2B, and MOB3/phocein) that form two functional NDR-MOB protein complexes. The MOB1-DBF2 complex is not only essential for septum formation in vegetative cells and during conidiation, but also functions during sexual fruiting body development and ascosporogenesis. The two MOB2-type proteins interact with both COT1 isoforms and control polar tip extension and branching by regulating COT1 activity. The conserved region directly preceding the kinase domain of COT1 is sufficient for the formation of COT1-MOB2 heterodimers, but also for kinase homodimerization. An additional $\mathrm{N}$-terminal extension that is poorly conserved, but present in most fungal NDR kinases, is required for further stabilization of both types of interactions and for stimulating COT1 activity. COT1 lacking this region is degraded in a mob-2 background. We propose a specific role of $\mathrm{MOB} 3 /$ phocein during vegetative cell fusion, fruiting body development and ascosporogenesis that is unrelated to the three other MOB proteins and NDR kinase signaling.
\end{abstract}




\section{Introduction}

Establishment of cell polarity and maintenance of cellular asymmetry are essential cellular properties that govern morphogenesis and development of uni- and multicellular organisms. Members of the conserved nuclear $\underline{D} b f 2 p$-related (NDR) kinase family are important for growth and differentiation in various organisms. In Drosophila melanogaster, the NDR kinase Tricornered is required for controlling cell proliferation as well as for neuronal morphogenesis (Emoto et al., 2004; Emoto et al., 2006; Geng et al., 2000; Justice et al., 1995; Wei et al., 2007; Xu et al., 1995), while the Caenothabditis elegans homolog SAX-1 regulates aspects of neuronal cell shape and has been proposed to be involved in cell spreading, neurite initiation and dendritic tiling (Gallegos and Bargmann, 2004; Zallen et al., 2000). Further work has resulted in an emerging NDR signaling network (Kanai et al., 2005; Nelson et al., 2003 summarized in Hergovich et al., 2006), in which NDR kinase activity is controlled by its binding partner MOB (Bichsel et al., 2004; Hergovich et al., 2005) and an upstream germinal center kinase of the Ste20 superfamily that controls NDR phosphorylation status and is required for its full activation (Stegert et al., 2005; Wei et al., 2007).

Although NDR pathway elements are highly conserved among eukaryotes, impairing their functions can result in highly divergent cellular responses, indicating that the detailed wiring of these components is critical for NDR signaling in a specific organism. This is best illustrated by the comparison of Cbk1p pathway mutants in Saccharomyces cerevisiae and Cryptococcus neoformans (called RAM mutants in these organisms for "regulation of Ace2p activity and cellular morphogenesis"). Mutations in RAM components in these two organisms result in either loss of cell polarity or in hyperpolarized growth, respectively (Nelson et al., 2003; Walton et al., 2006).

Phylogenetic analyses support the presence of a second group of NDR kinases in all eukaryotes called Dbf2p and SID2 in budding and fission yeast, respectively. The activity of both kinases requires binding of MOB1, several upstream protein kinases and a Rassuperfamily GTPase (Krapp and Simanis, 2008; Walther and Wendland, 2003; Wolfe and Gould, 2005). The function of this network, called septation initiation network (SIN) in fission yeast or mitotic exit network (MEN) in budding yeast, is the coordination of nuclear division with cytokinesis. The unifying feature of mutants in SIN components is 
defective septum formation and the result are aseptate strains. S. cerevisiae MEN mutants behave slightly different and arrest as dumbbell shaped cells, indicative of late telophase arrest. Thus, each NDR kinase pathway in the two yeasts has clearly defined and separate functions and either connects mitotic exit with cytokinesis or is involved in polarity and cellular morphogenesis, respectively (Kanai et al., 2005; Krapp and Simanis, 2008; Nelson et al., 2003).

This clear separation between cell cycle and morphogenetic functions of NDR signaling is not conserved in animals, and the number of NDR kinases and of MOB adapter proteins and thus of potential MOB-NDR kinase interaction pairs has increased with the increasing complexity of the organism. Filamentous fungal genomes contain two ndr and up to four mob genes (Galagan et al., 2003; Galagan et al., 2005), while four NDR kinases (representing two kinases in each major subgroup) and six MOB proteins are present in vertebrates (Bichsel et al., 2004; Devroe et al., 2004). Each kinase has been found to be able to associate with several MOB proteins in higher eukaryotes, and the distinction between cell cycle control and morphogenesis is less strict than in yeasts (Bichsel et al., 2004; He et al., 2005b; Hergovich et al., 2005; Lai et al., 2005).

Despite the relevance of an apically growing tip cell for most members of the fungal kingdom, the key components that are required for tip extension and for colonization of substrates are poorly understood. Furthermore, NDR pathway components have been shown to be essential for pathogenicity and virulence in all fungal pathogens analyzed so far (Durrenberger and Kronstad, 1999; McNemar and Fonzi, 2002; Scheffer et al., 2005; Walton et al., 2006). To date, the protein kinase COT1 of Neurospora crassa, the founding member of the NDR kinase family (Yarden et al., 1992), and POD6, a germinal center kinase, which is associated with COT1 (Maerz et al., 2008; Seiler et al., 2006), are among the best-characterized components that specifically regulate tip growth and branch formation, but not cell polarity per se. Temperature-sensitive mutants of these two kinases cease hyphal elongation with a needle-shaped apex at restrictive temperature and produce massive amounts of extension-arrested new tips along the entire cell. Strains in which these genes have been deleted are viable and form compact colonies with growth-arrested tips, indicating that both kinases are essential for tip extension and for restricting supernumerary branch formation, but are not required for establishing new sites of growth (Collinge and Trinci, 1974; Collinge et al., 1978; Seiler and Plamann, 2003; 
Seiler et al., 2006; Yarden et al., 1992). cot-1 encodes for two transcripts, whose ratio of expression is photo-regulated, but the functional significance of the two generated COT1 isoforms that differ only in their N-terminus remains undetermined (Gorovits et al., 1999; Lauter et al., 1998).

The importance of NDR signaling for fungal growth and the subtle differences in the wiring of these elements has prompted us to dissect the function and nature of interactions between NDR kinases and MOB proteins in more detail. We show that the MOB1-DBF2 and MOB2-COT1 complexes function as distinct modules during septation and tip growth. MOB3/phocein, however, is specific for vegetative cell fusion and fruiting body development and is unrelated to NDR kinase signaling. 


\section{Results}

\section{Three types of MOB proteins with distinct functions are present in filamentous fungi}

Database searches identified four MOB proteins in the genome of $N$. crassa that were most similar to fungal MOB1 and MOB2 and to the more distantly related MOB family member MOB3/phocein (Figure III-1).
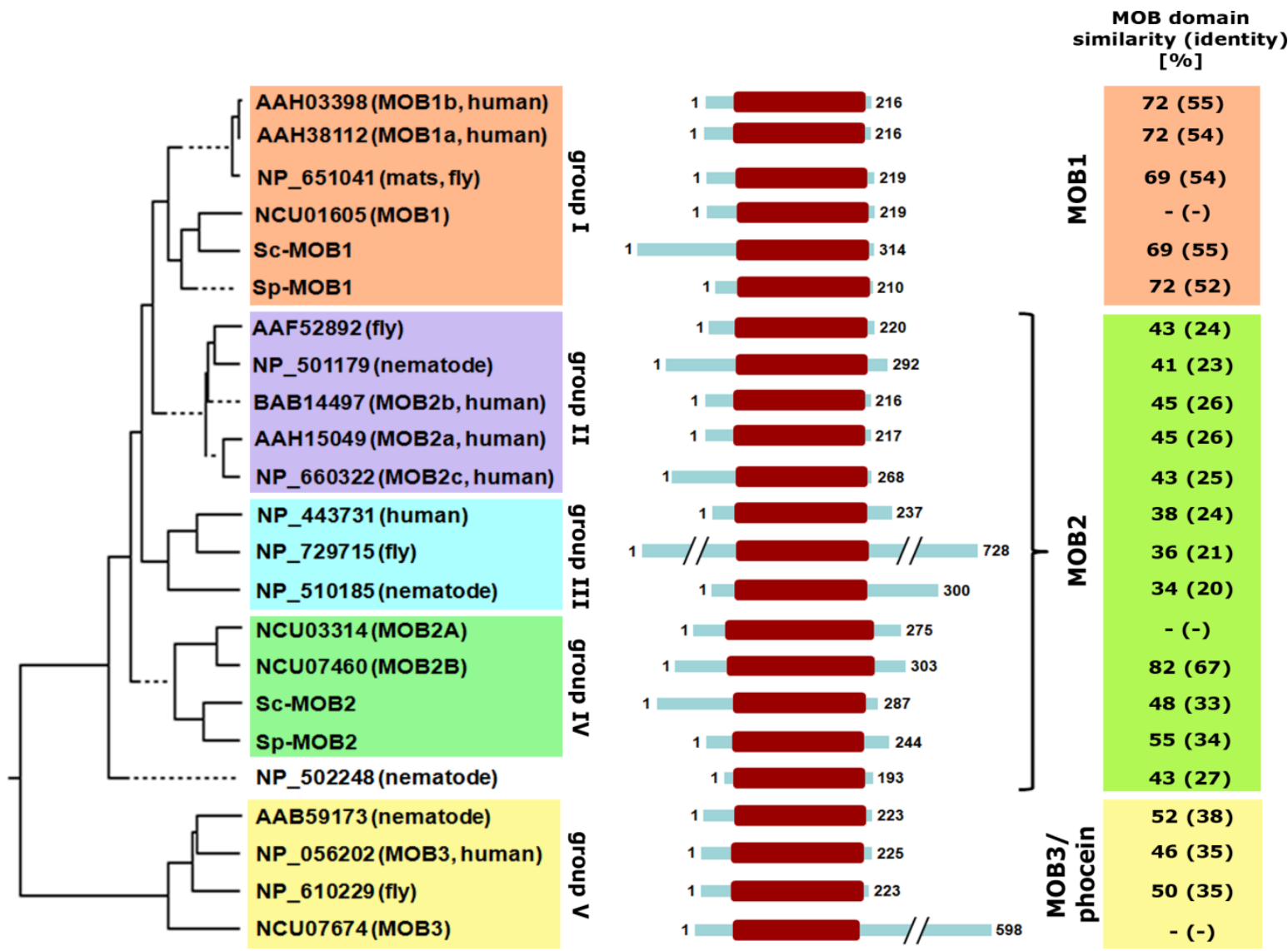

Figure III-1 Phylogenetic distribution of the MOB protein family. MOB proteins of budding and fission yeast (named ScMOB and SpMOB, respectively), N. crassa (BROAD accession numbers NCUxxxx) and selected animal MOBs (NCBI accession numbers) were aligned using the MegAlign program from DNAStar (Lasergene) to generate a phylogenetic tree based on the Clustal V (PAM 250) method. MOB1 and MOB3/phocein proteins form distinct subgroups, while MOB2-type proteins separate into two animal specific clusters and a fungal specific group. The C. elegans protein NP_502248 is likely a distinct member of the MOB1 group. Schematic diagrams of individual MOB proteins depict the conserved MOB domain. On the right, the percentage of sequence identity and similarity between the MOB domains and the respective N. crassa MOB protein in each subgroup is indicated. 
Based on these sequence similarities, NCU01605 was designated mob-1, NCU03314 and NCU07460 mob-2a and mob-2b, respectively, and NCU07674 mob-3. Sequence comparisons within the available fungal genomes revealed the presence of at least one MOB protein of each type in all filamentous growing members of the fungal kingdom. MOB3/phocein, however, was detected only in filamentous fungi and higher eukaryotes, but not in unicellular yeasts.

Strains harboring deletions of the four mob genes were provided by the N. crassa genome project (Dunlap et al., 2007) and were used to determine cellular functions of the different MOB proteins (Figure III-2). $\Delta m o b-1$ was characterized by a growth rate that was reduced to $40 \%$ of wild type, increased branch formation and a strong cell lysis defect. The generation of aerial mycelium was abolished and conidiation was reduced to $<1 \%$ of wild type. Furthermore, $\Delta m o b$-1 was unable to generate female reproductive structures. This inability to form protoperithecia may be seen as a secondary consequence of the described vegetative defects, but fertilization of wild type with heterokaryotic $\Delta m o b-1+$ $m o b-1^{+}$conidia resulted in defective ascosporogenesis and the frequent formation of asci containing only a single, large, ascospore. These giant ascospores produced colonial growth similar to wild type on selective medium, indicating that the deletion phenotype was sheltered by $m o b-1^{+}$in these progeny and that MOB1 is directly involved in meiosis and ascosporogenesis.

$\Delta m o b-2 a$ and $\Delta m o b-2 b$ displayed similar defects, which were less pronounced than those of $\Delta m o b-1$. The deletions resulted in slightly reduced growth rates accompanied by increased branching frequencies, with $\Delta m o b-2 a$ being more compromised than $\Delta m o b-2 b$ (70\% and $92 \%$ of the wild type growth rate, respectively). Furthermore, we observed altered aerial hyphae formation and the generation of reduced amounts of conidia ( $11 \%$ and $54 \%$ of wild type, respectively). The sexual development of $\Delta m o b-2 a$ and $\Delta m o b-2 b$ was not affected, and the strains were female fertile and generated abundant, normally shaped ascospores in $\Delta \mathrm{x}$ wild type and in $\Delta \mathrm{x} \Delta$ crosses with ascospore germination rates that were indistinguishable from wild type. 
A
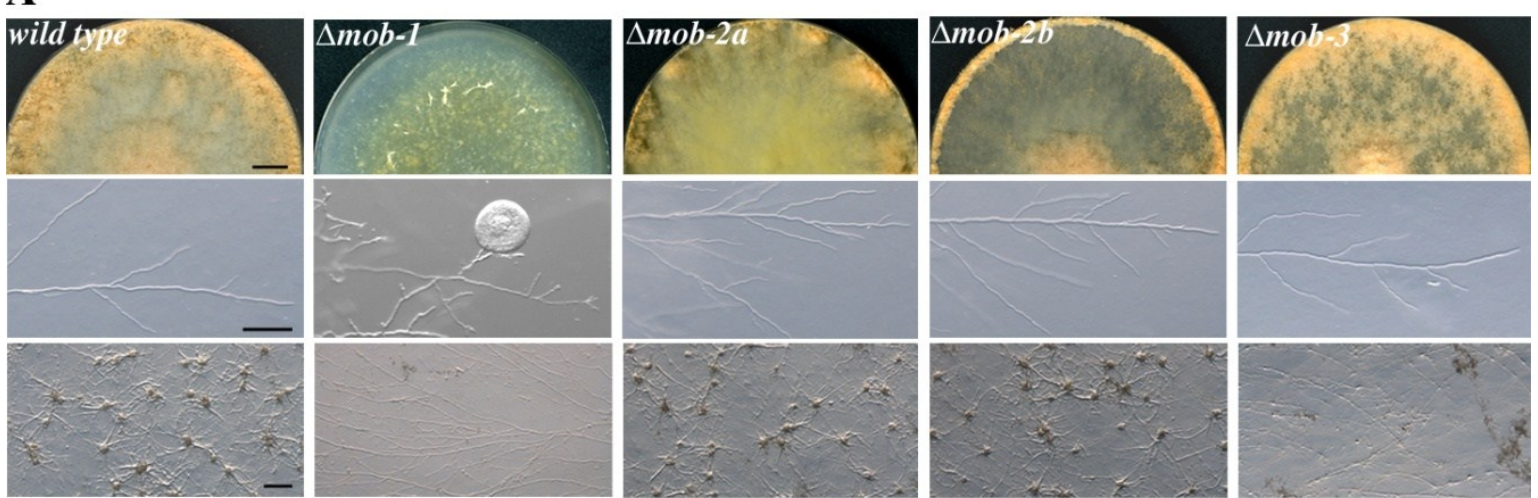

B
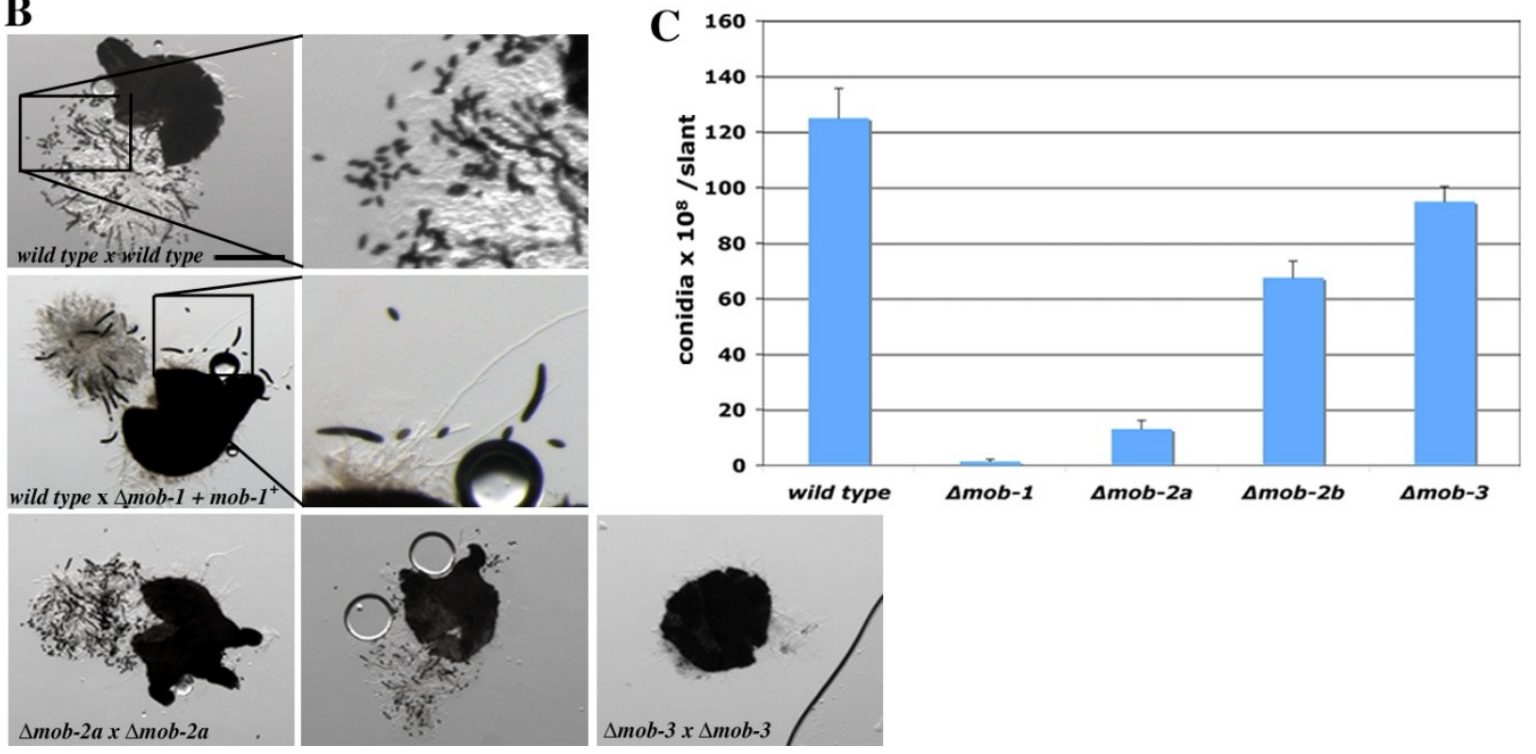

Figure III-2 MOB proteins have distinct cellular function in $\mathbf{N}$. crassa. (A) Phenotypic characterization of the indicated mob deletion strains with regard to colony morphology (upper panel; growth for $5 \mathrm{~d}$ on minimal medium; bar $=1 \mathrm{~cm}$ ), hyphal morphology on minimal medium (middle panel; bar $=50 \mu \mathrm{m}$ ), and protoperithecia formation; (lower panel; growth for $7 \mathrm{~d}$ on cornmeal medium; bar $=300 \mu \mathrm{m}$ ). (B) Ascus development three weeks after fertilization with the indicated male partner in squeezed perithecia of the indicated crosses (bar $=100 \mu \mathrm{m}$ ); note the presence of large banana-shaped ascospores in the $\Delta m o b-1$ cross. (C) Production of conidiospores was quantified by counting conidia generated in slants grown at room temperature for $5 \mathrm{~d}(\mathrm{n}=5$; standard deviations are indicated as bars).

The growth rate of $\Delta m o b-3$ was almost as high as the wild type (89\%). Conidial production was only mildly affected ( $76 \%$ of wild type) and probably a consequence of reduced aerial mycelium formation. This contrasted with a ca. 30-fold reduction in the number of protoperithecia produced by $\Delta m o b-3$. Furthermore, the few protoperithecia produced were much smaller and less developed than in the wild type. When these $\Delta m o b-3$ protoperithecia were fertilized with wild type or $\Delta m o b-3$ conidia, further development was blocked and only empty perithecia were formed that lacked ascogenous hyphae and 
developing asci. Wild type protoperithecia that were fertilized with $\Delta$ mob-3 conidia developed further, but also resulted in only very few viable ascospores. Such defects in fruiting body formation and sexual development have been frequently connected with a failure of vegetative cell fusion (Fleissner et al., 2008; Maerz et al., 2008; Wei et al., 2003). When we compared the ability to undergo cell fusion in germlings and mature hyphae of $\Delta m o b-3$ and wild type (Figure III-3), cell fusion was readily visible in wild type under both conditions using conventional light microscopy, but we were unable to detect fusion events in $\Delta m o b-3$, indicating that cell fusion is dependent on MOB3 function. These assays do not rule out that cell fusion may occur at significantly lower frequencies, which may be suggested by the limited formation of fertile perithecia in wild type $\mathrm{x} \Delta$ mob-3 crosses. However, it is worth noting that the capability for and mechanistic nature of selfself fusion (which was assayed in the microscopic tests) may not be identical to selfnonself fusion (detected in the cross).
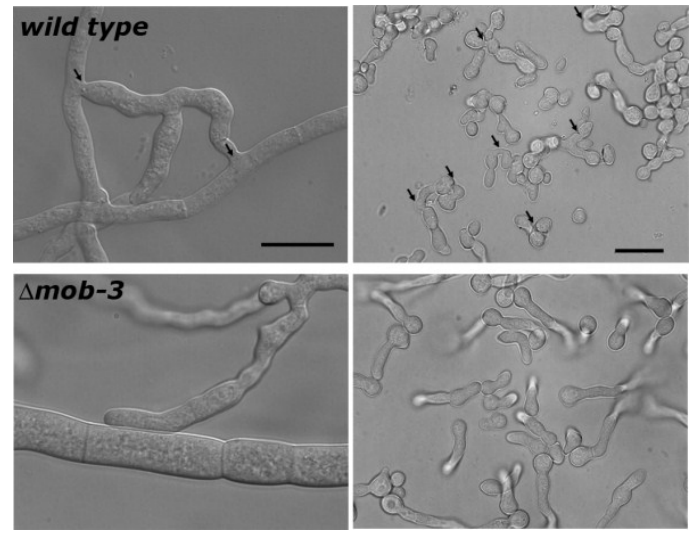

Figure III-3 МОВ3 is required for vegetative cell fusion. Hyphal fusion (left images; bar = $10 \mu \mathrm{m}$ ) and germling fusion (right images; bar $=5 \mu \mathrm{m}$ ) in wild type and $\Delta m o b-3$ cultures was assessed by light microscopy. Fusion events are indicated by arrows. Cell fusion was not observed in $\Delta m o b-3$.

\section{MOB1-DBF2 and MOB2A/2B-COT1 complexes function as distinct modules during septation and tip growth}

To test for potentially redundant functions of the four MOB proteins, we generated double mutants. No obvious synthetic interaction was observed in $\Delta m o b-1 ; \Delta m o b-2 a$ and $\Delta m o b-1 ; \Delta m o b-2 b$, and both strains displayed lysing hyphae as their most characteristic phenotype (data not shown). $\Delta m o b-2 a ; \Delta m o b-2 b$ however, formed tight hyperbranching colonies with extension-arrested tips, a phenotypic trait highly reminiscent to conditional 
or deletion mutants of both kinases of the COT1 complex (cot-1 and pod-6) germinating at restrictive temperature (Figure III-4 A). We did not detect synthetic defects in any mob double mutant combinations containing $\Delta m o b$-3. Thus, we propose MOB3 has a specific role during vegetative cell fusion and sexual development that is unrelated to the functions of the three other MOB proteins.

The mutant characteristics of $m o b$ and $n d r$ deletion strains suggested specific interactions between $\mathrm{COT} 1$ and $\mathrm{MOB} 2$ proteins and $\mathrm{DBF} 2$ and MOB1. To further test these possible interactions, we generated strains harboring tagged versions of both NDR kinases to identify copurifying proteins. A myc-dbf-2-containing construct ectopically integrated in $\Delta d b f-2 ;$ his-3 complemented the septation defect, indicating functionality of the fusion protein. In addition, we generated a strain, in which a 6xmyc tag was inserted in-frame at the second ATG of the endogenous cot-1 locus, which allowed the simultaneous detection of both COT1 isoforms (designated myc-cot-1). The wild type growth of this strain demonstrated the functionality of the modified endogenous cot-1 allele. Products of protein immunoprecipitation (IP) were resolved by SDS-PAGE, and specific bands were excised from gels and analyzed by mass spectrometry (Figure III-4 C). We observed two bands of proteins of ca. 30 and $38 \mathrm{kDa}$ that were consistently associated with myc-COT1 and identified them as MOB2A and MOB2B, respectively. The interaction of MOB2A with COT1 was tighter than that with MOB2B, as we repeatedly detected reduced amounts of MOB2B, but never of MOB2A in our IPs. MOB1 could not undoubtedly be identified in myc-DBF2 precipitants by LC-MS (see Material and Methods for criteria of LC-MS identification). Other copurifying proteins were also detected, in varying amounts, in IPs using untagged wild type, and are, most likely, contaminants. 

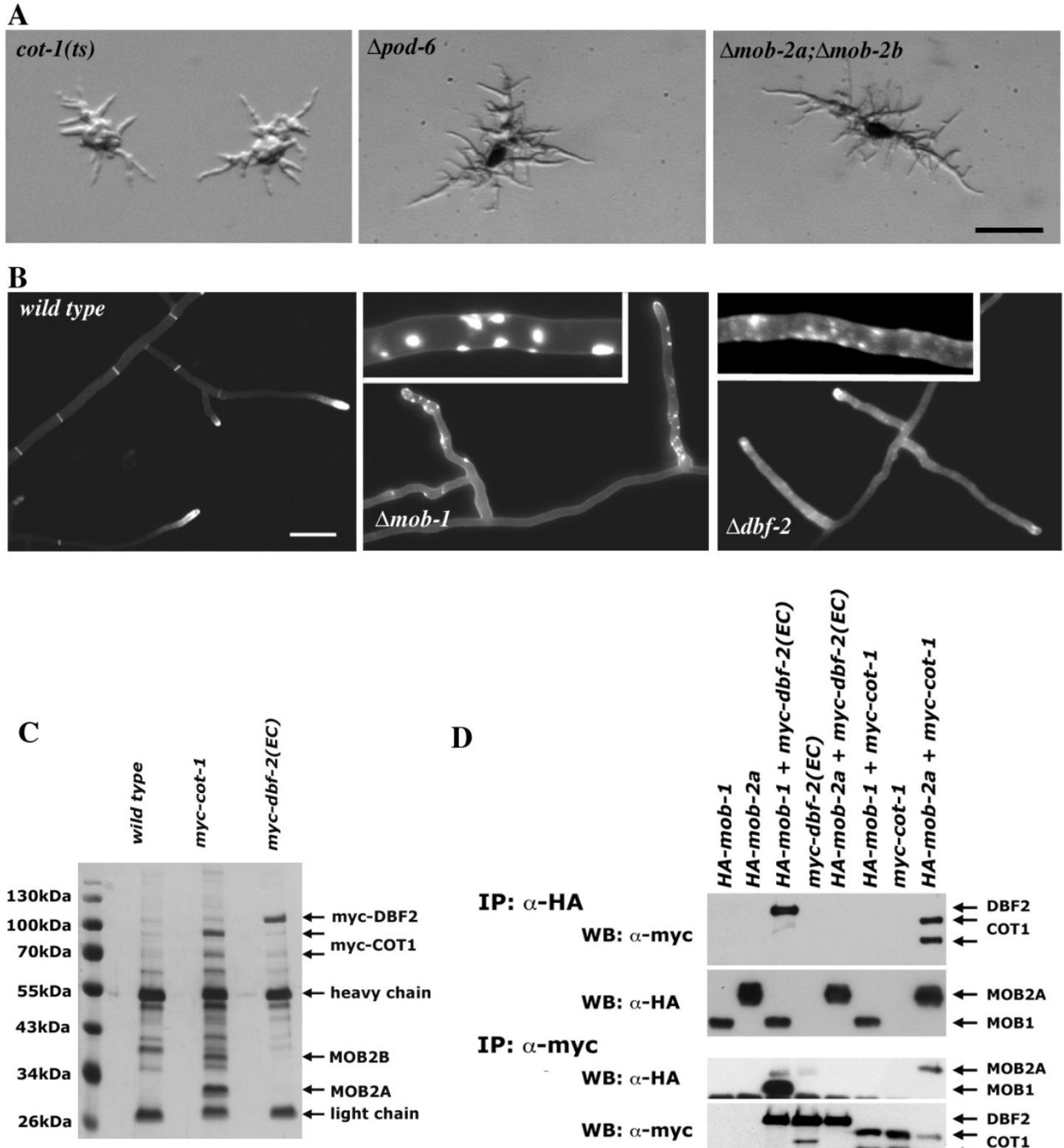

D

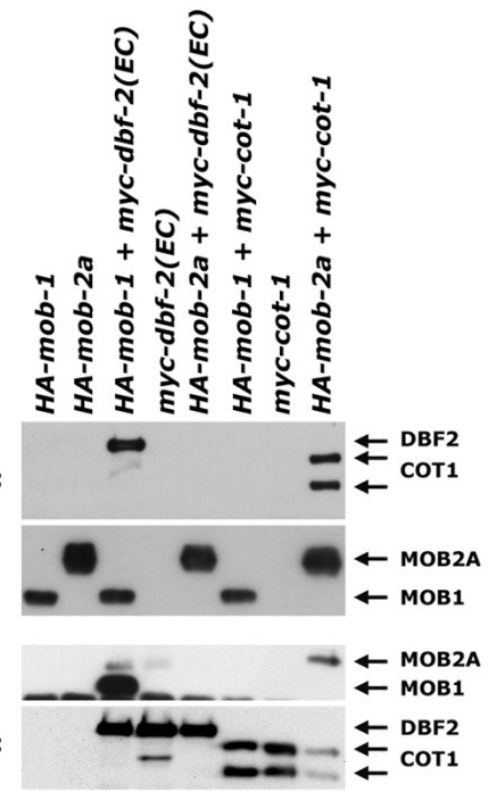

Figure III-4 MOB1-DBF2 and MOB2A/2B-СOT1 function as distinct modules during septation and tip growth. (A) Asco- or conidospores of the indicated mutants were germinated for $2 \mathrm{~d}$ or $1 \mathrm{~d}$, respectively, on minimal medium supplemented with hygromycin at $37^{\circ} \mathrm{C}(\mathrm{bar}=5 \mu \mathrm{m})$. (B) Calcofluor White staining indicated aberrant septation and the lack of functional cross walls in $\Delta m o b-1$ and $\Delta d b f-2$ (bar $=10 \mu \mathrm{m}$ ). Stainable cell wall material accumulated in a patchy manner in both mutants, but was more prominent in $\Delta m o b$-1 than in $\Delta d b f-2$. (C) Copurification of myc-DBF2 and myc-COT1 associated proteins from the indicated strains. (D) Immunoprecipitation (IP) experiments with anti-HA and anti-MYC antibodies and subsequent Western blot analysis (WB) from the indicated strains. 
The identification of DBF2-associated MOB1 by LC-MS analysis was challenging, as the antibody light chain used for the IP experiments had the same molecular weight as predicted for MOB1. To overcome this difficulty and to further test for the specificity of the NDR - MOB interactions, we expressed HA-tagged versions of mob-1 and mob-2a in a nic-3 strain. Furthermore, myc-cot-1 was crossed into a his-3 background and myc-dbf-2 ectopically integrated in a his-3 strain. Various combinations of forced heterokaryons, capable of growing on minimal medium by complementation of the individual strains' auxotrophies were then generated and subsequently used for co-IP experiments. We detected interactions of COT1 with MOB2A and of DBF2 with MOB1, but not between COT1 and MOB1 or DBF2 and MOB2A (Figure III-4 D). Thus, the interaction profile of NDR kinases and MOBs and the phenotypic characteristics of the individual ndr and mob deletion strains indicated the existence of two distinct NDR-MOB complexes consisting of MOB1 associated with DBF2 and MOB2A and MOB2B bound to COT1.

\section{MOB2 proteins affect kinase activity and COT1 stability}

To determine the relative impact of individual MOB2 proteins on COT1 function, we generated double mutants of mob deletions with conditional cot-1(ts) and pod-6(ts) strains and assayed them for synthetic growth defects at different temperatures (Table III-1). Strong synthetic interactions were detected for $\Delta m o b-2 a ; \cot -1$ (ts) and $\Delta m o b$ 2a;pod-6(ts). Their growth rates were reduced to $53 \%$ and $55 \%$ at permissive temperature, and to $4 \%$ and $43 \%$ under semipermissive conditions, respectively, compared to the slower growing parental strains. Moreover, $\Delta m o b-2 a ; \cot -1$ (ts) developed the typical cot-like hyperbranched colonies at semipermissive conditions, indicating the importance of MOB2A for COT1 function. Synthetic interactions were also detected for double mutants of $\Delta m o b-1$ and $\Delta m o b-2 b$ with both conditional kinase strains at semipermissive temperatures. We did not detect any synthetic interactions of cot-1(ts) or pod-6(ts) with $\Delta m o b-3$, a further indication for the lack of related functions between MOB3 and NDR signaling pathways. 
Table III-1 Radial growth rates of COT1 complex single and double mutants ( $n \geq 3$ )

\begin{tabular}{|c|c|c|c|c|c|}
\hline & \multicolumn{3}{|c|}{ growth rate $(\mathrm{cm} /$ day $\pm S D)$} & \multicolumn{2}{|c|}{$\begin{array}{c}\text { relative growth (\% of slower } \\
\text { parental strain) }\end{array}$} \\
\hline & \multirow[t]{2}{*}{ permissive* } & \multicolumn{2}{|c|}{ semipermissive* } & \multirow[t]{2}{*}{ permissive* } & \multirow[t]{2}{*}{ semipermissive* } \\
\hline & & $30^{\circ} \mathrm{C}$ & $34^{\circ} \mathrm{C}$ & & \\
\hline $\cot -1(t s)$ & $3.0 \pm 0.3$ & $2.5 \pm 0.1$ & & / & / \\
\hline pod-6 (ts) & $3.1 \pm 0.1$ & & $3.7 \pm 0.2$ & / & / \\
\hline$\Delta m o b-1$ & $1.4 \pm 0.1$ & $2.7 \pm 0.2$ & $2.6 \pm 0.2$ & / & / \\
\hline $\cot -1(t s) ; \Delta m o b-1$ & $1.3 \pm 0.1$ & $0.6 \pm 0.3$ & & 93 & 25 \\
\hline pod-6(ts); $\Delta m o b-1$ & $1.4 \pm 0.1$ & & $0.8 \pm 0.1$ & 100 & 30 \\
\hline$\Delta m o b-2 a$ & $2.1 \pm 0.2$ & $3.9 \pm 0.2$ & $4.5 \pm 0.1$ & / & / \\
\hline $\cot -1(t s) ; \Delta m o b-2 a$ & $1.1 \pm 0.1$ & $0.1 \pm 0$ & & 53 & 4 \\
\hline pod-6(ts); $\Delta m o b-2 a$ & $1.2 \pm 0.1$ & & $1.6 \pm 0.2$ & 55 & 43 \\
\hline$\Delta m o b-2 b$ & $3.2 \pm 0.2$ & $5.2 \pm 0.2$ & $5.4 \pm 0.2$ & / & / \\
\hline $\cot -1(t s) ; \Delta m o b-2 b$ & $2.6 \pm 0.3$ & $2 \pm 0.3$ & & 87 & 82 \\
\hline$p o d-6(t s) ; \Delta m o b-2 b$ & $2.1 \pm 0.2$ & & $2.5 \pm 0.3$ & 68 & 68 \\
\hline$\Delta m o b-3$ & $3.1 \pm 0.2$ & $4.7 \pm 0.1$ & $5.6 \pm 0.2$ & / & / \\
\hline $\cot -1(t s) ; \Delta m o b-3$ & $2.8 \pm 0.1$ & $2.1 \pm 0.2$ & & 95 & 86 \\
\hline pod-6(ts); $\Delta m o b-3$ & $3.1 \pm 0.1$ & & $3.2 \pm 0.2$ & 100 & 89 \\
\hline
\end{tabular}

Next, we generated $\Delta m o b$ strains, in which cot-1 was myc-tagged at the endogenous locus, to quantify the impact of individual MOBs on COT1's kinase activity by crossing the deletion strains with myc-cot-1. In contrast to myc-COT1 precipitated from myc-cot-1, which displayed robust in vitro kinase activity, myc-COT1 purified from myc-cot-1; $\Delta m o b$ $2 a$ and $m y c-c o t-1 ; \Delta m o b-2 b$ showed activities that were reduced to $45 \pm 3 \%$ and $80 \pm 17 \%$ (n = 3), respectively (Figure III-5 A). The copurification of both MOB2 proteins and their double mutant phenotype suggested overlapping functions of the two MOBs. Thus, we performed kinase assays with precipitants of a $\Delta m o b-2 a ; \Delta m o b-2 b ; m y c-c o t-1$ strain. We were able to precipitate myc-СОT1 from these poorly growing cultures. The detected in vitro activity was only barely above background $(1.0 \pm 0.5 \%$ of myc-COT1; $n=3)$, indicating that the presence of the two MOB2 proteins is a prerequisite for in vitro COT1 activity and in vivo COT1 function. 
We found that the small myc-СОT1 isoform in the precipitant was almost absent from $\Delta m o b-2 a ; \Delta m o b-2 b ; m y c-c o t-1$ precipitant. To determine if this was due to the loss of kinase activity or to the absence of the MOB proteins, we examined the presence of the large and small COT1 isoforms in cell extracts of $\Delta m o b-2 a ; \Delta m o b-2 b ; m y c-c o t-1$ (Figure III-5 B). As a control, we generated a kinase dead strain, in which the catalytic aspartate 337 was mutated to alanine. myc-cot-1(D337A) displayed highly compact growth and hyperbranching defects, which were undistinguishable from $\Delta$ cot-1 or $\Delta$ pod-6 cells (Figure III-5 C). When we compared these extracts, we found that the degradation of COT1's smaller isoform was due to the absence of MOB2 proteins and not due to the lacking kinase activity.
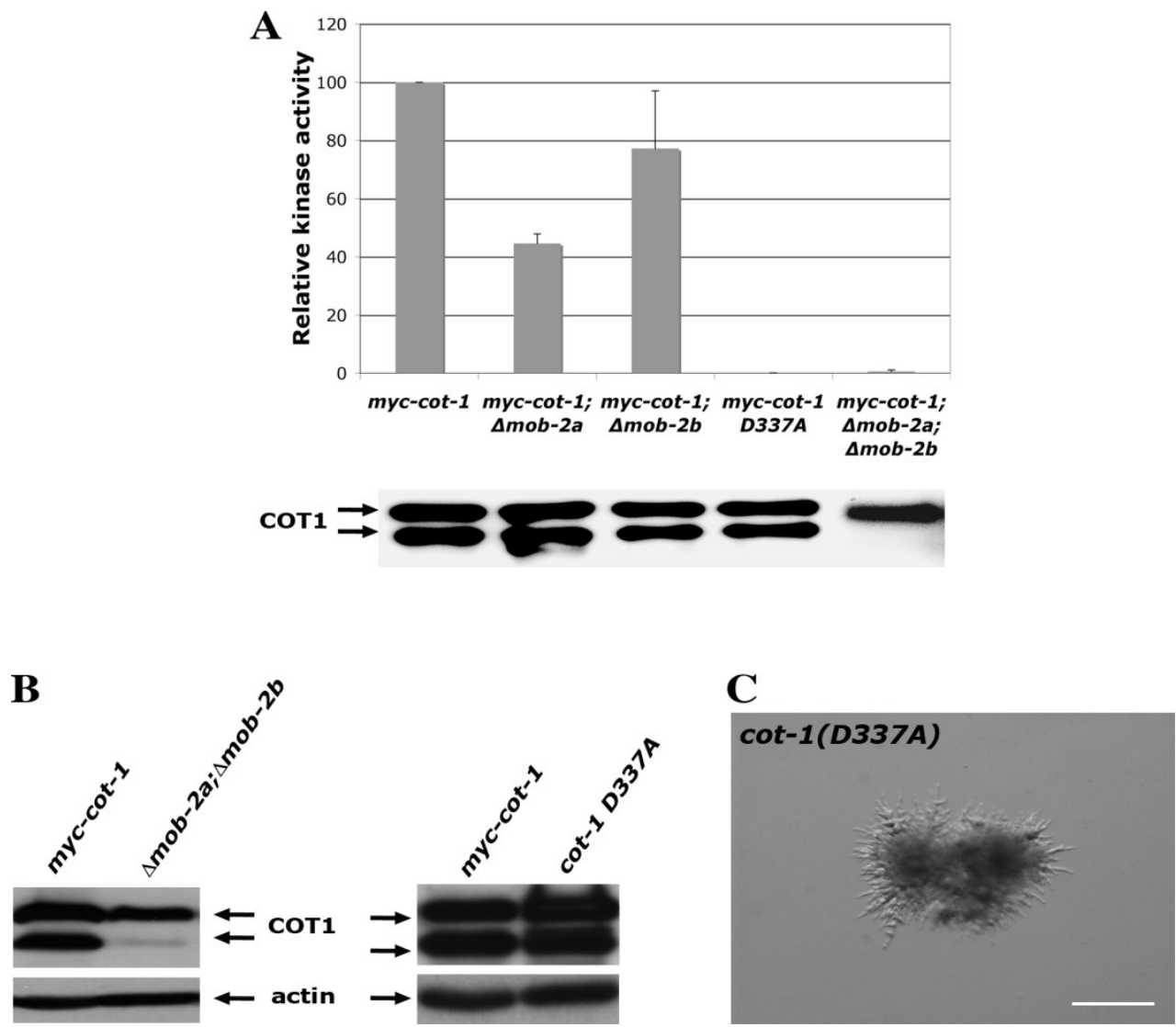

Figure III-5 МОВ2 proteins are essential for СОТ1 function. (A) Kinase activity of myc-СОT1 purified from the indicated mutants and the catalytically inactive strain cot-1(D337A). Data represent means of at least four independent experiments with $\geq 3$ independent clones of each mutant (standard deviations are indicated as bars). (B) The abundance of both COT1 isoforms in cell extracts of the indicated strains was determined by Western Blot analysis with anti-myc antibody and probed with an anti-actin antibody as control. (C) The kinase dead cot-1(D337A) strain has morphological defects indistinguishable from $\Delta c o t-1$. 


\section{MOB2 binding is required, but not sufficient for COT1 activation}

The presence of two MOB2-type proteins that interact with myc-COT1, and the fact that two isoforms of COT1 are expressed in $N$. crassa that differ by an 118 amino acid $\mathrm{N}$ terminal extension of unknown function (Gorovits et al., 1999) led us to ask if a specific MOB2 protein interacted with each COT1 isoform. We generated two cot-1 constructs, in which a 3xmyc-6xhis tag was fused with the first or second ATG of cot-1, respectively. Expression of myc-COT1(long) or myc-COT1(short) was controlled by the inducible qa-2 promoter, and the constructs were ectopically inserted at the his-3 locus of a cot-1(ts); his3 strain. Both constructs complemented the temperature-sensitive growth defects in the presence of $10 \mathrm{mM}$ quinic acid, indicating that each isoform did substitute for all COT1 functions defective in the temperature-sensitive strain. These strains were used for single step anti-myc co-IP and tandem his-myc affinity purification experiments (Figure III-6 A). Both purification conditions resulted in the copurification of both MOB2 proteins with the long and the short COT1 isoform, indicating that the $\mathrm{N}$-terminal extension did not specify the interaction with $\mathrm{MOB2A}$ or МОВ2B. Although we observed variable amounts of coprecipitated МOB2B from both extracts, this variability was not significantly different from control-IPs from myc-cot-1 cultures, and the total amount of coprecipitated MOB corresponded to the level of precipitated COT1 $(n=3)$. We then used IP products of cultures expressing the two isoforms to determine their in vitro kinase activities (Figure III-6 B). myc-COT1(long) displayed $45 \pm 4 \%$ of myc-COT1 activity, which was consistent with the presence of only one isoform and thus of $56 \pm 19 \%$ precipitated myc-COT1(long) compared to myc-COT1 ( $\mathrm{n}=3$ ). myc-COT1(short) activity, however, was reduced to $33 \pm 4 \%$ of myc-COT1(long) and to $15 \pm 2 \%$ of myc-COT1, despite the fact that its protein level was only reduced to $94 \pm 31 \%$ and $45 \pm 5 \%$ of myc-COT1(long) and myc-COT1, respectively. Thus, the $\mathrm{N}$-terminal extension of myc-COT1 is involved in the stimulation of the kinase. 


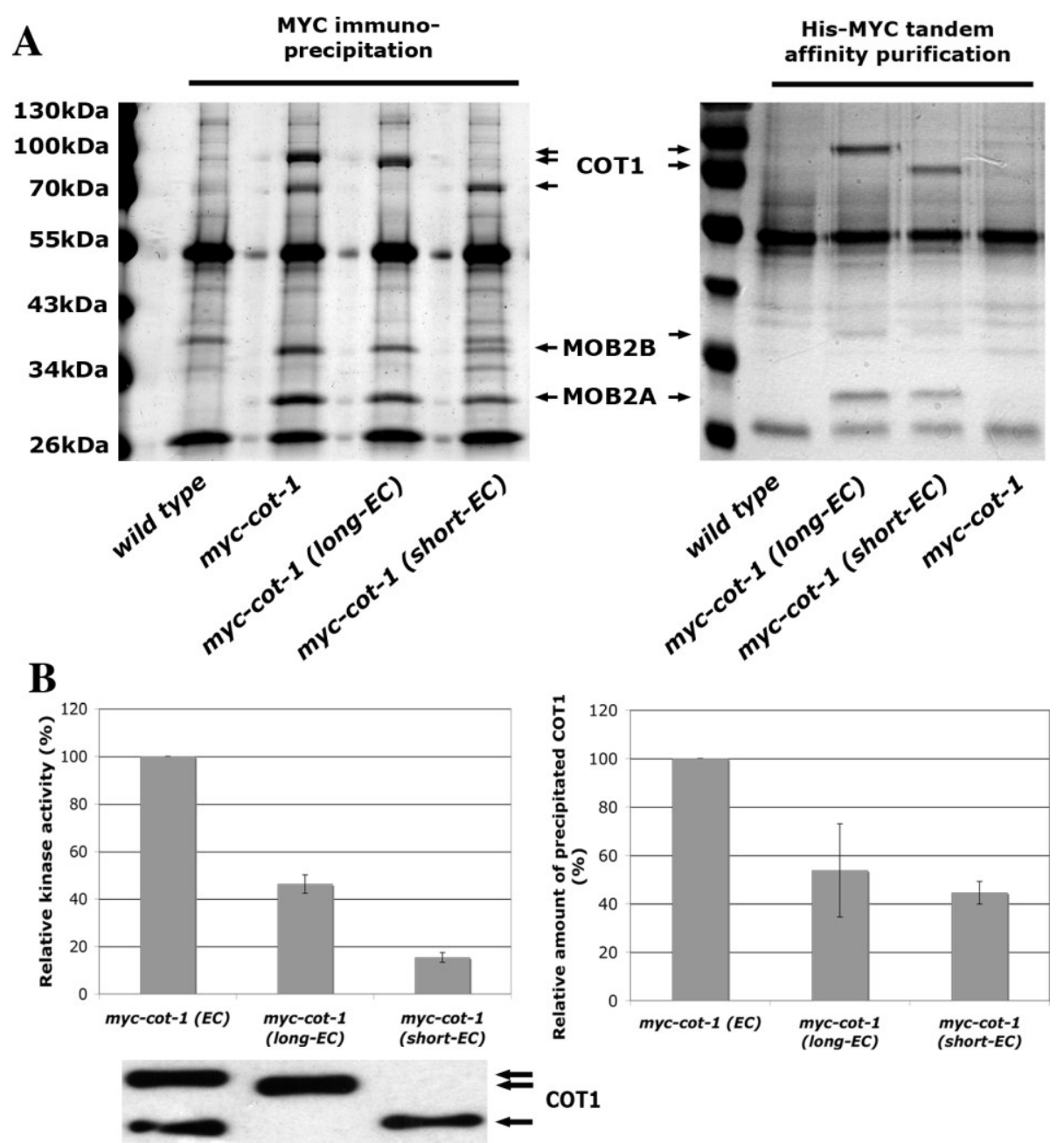

Figure III-6 Functional characterization of the two COT1 isoforms. (A) Single myc and double myc-his purification experiments of the individual СOT1 isoforms from the indicated strains (note that endogenously tagged myc-cot-1 is modified only by a 5xmyc-tag, while the ectopically integrated constructs are expressed as myc-his-fusion proteins). (B) Quantification of in vitro kinase activities (left diagram) and amounts of precipitated myc-COT1 (right diagram) from the strains expressing the individual isoforms ( $n=3$; standard deviations are indicated as bars).

\section{COT1 dimerization and interaction with MOB requires overlapping regions}

The binding of MOB to NDR requires the N-terminus of the kinase (Bichsel et al., 2004; He et al., 2005b; Song et al., 2008). In addition to this proposed MOB binding interface that is conserved in fungal and animal NDRs, most fungal NDR kinases contain an uncharacterized N-terminal extension of varying length. Sequence analysis of the available fungal proteins revealed the existence of homology groups that differ in this $\mathrm{N}$ terminal extension (supplementary Figure S III-1). S. pombe ORB6 is a representative of an NDR kinase that, similar to animal counterparts, has no N-terminal extension. This 
contrasts with S. cerevisiae Cbk1p that has a 260 amino acid extension lacking sequence homology to other proteins. However, a conserved feature of the Cbk1p extension that is shared with NDR kinase extensions in filamentous ascomycetes is its high content of asparagine and glutamine residues. In addition, NDR kinases of filamentous ascomycetes share conserved sequence motifs. For example, a methionine corresponding to the start codon of the short COT1 isoform is present in several (but not all) species, suggesting that the existence of multiple isoforms may be beneficial for filamentous growth.

Thus, we analyzed the sequence requirements for the COT1-MOB2 interaction in more detail. First, we confirmed the interaction of COT1 with both MOB2 proteins by yeast two hybrid assays (Figure III-7 A). Furthermore, we detected two hybrid interactions between COT1 and POD6 and observed dimerization of COT1. No interaction of POD6 with any of the $\mathrm{MOB}$ proteins and between the two $\mathrm{MOB}$ proteins was detected in these assays. We then determined which domain of COT1 is required for MOB binding by analyzing the $\mathrm{N}$ terminal regions of the two COT1 isoforms (amino acids 1-212 and 119-212; designated long and short, respectively; Figure III-7 B). Specific interactions were detected between both COT1 fragments and MOB2A, indicating that region 119-212 of COT1 is sufficient to interact with MOB proteins. In addition, we found that the long fragment also interacted with MOB2B. Moreover, growth curves of two hybrid cultures under selective conditions revealed a stronger interaction of MOB2A with the COT1 region 1-212 than with the region 119-212 (Figure III-7 C). These data are consistent with our biochemical purifications that showed tight binding of MOB2A with myc-COT1 and a more variable interaction between myc-СОT1 and МOB2B (Figures III-4 C and III-6 A).

Because COT1 dimerized in the two hybrid assays, we also examined which region of COT1 is required for self-association (Figure III-7 B, C). Both N-terminal COT1 fragments interacted with full length COT1, yet the interaction of COT1 was stronger with fragment 1-212 than with fragment 119-212. Self association was also detected between the two long COT1 fragments, but not when the two short fragments were used, indicating that region $119-212$ is sufficient for homodimerization, but also that the interaction is stabilized by amino acids 1-118. 
A

\begin{tabular}{|c|c|c|}
\hline $\begin{array}{c}\text { positive } \\
\text { control }\end{array}$ & $\begin{array}{c}\text { negative } \\
\text { control }\end{array}$ & $\begin{array}{c}\text { COT1 } \\
+ \\
\text { COT1 }\end{array}$ \\
\hline $\begin{array}{c}\text { COT1 } \\
+ \\
\text { POD6 }\end{array}$ & $\begin{array}{c}\text { COT1 } \\
+ \\
\text { MOB2A }\end{array}$ & $\begin{array}{c}\text { COT1 } \\
+ \\
+ \\
\text { MOB2B }\end{array}$ \\
\hline $\begin{array}{c}\text { COT1 } \\
+ \\
\text { pGADT7 }\end{array}$ & $\begin{array}{c}\text { pGBKT7 } \\
+ \\
\text { COT1 }\end{array}$ & $\begin{array}{c}\text { pGBKT7 } \\
+ \\
\text { POD6 }\end{array}$ \\
\hline $\begin{array}{c}\text { PGBKT7 } \\
+ \\
\text { MOB2A }\end{array}$ & $\begin{array}{c}\text { pGBKT7 } \\
+ \\
\text { MOB2B }\end{array}$ & \\
\hline
\end{tabular}
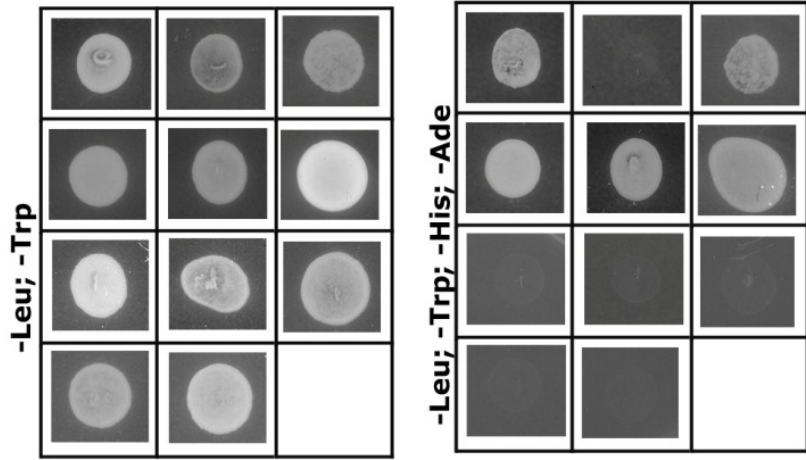

B

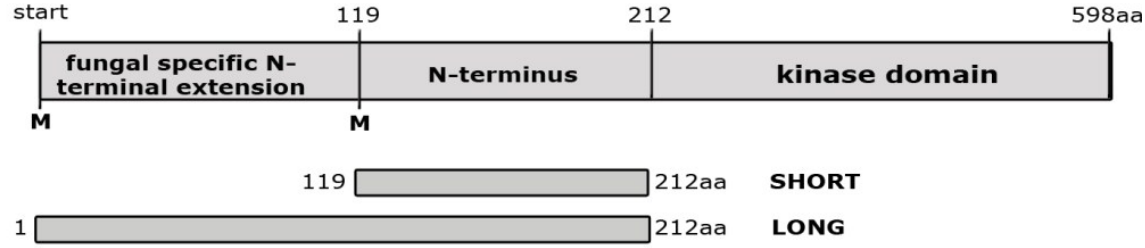

\begin{tabular}{|c|c|c|}
\hline $\begin{array}{c}\text { LONG } \\
+ \\
\text { LONG }\end{array}$ & $\begin{array}{c}\text { SHORT } \\
+ \\
\text { SHORT }\end{array}$ & $\begin{array}{c}\text { LONG } \\
+ \\
\text { COT1 }\end{array}$ \\
\hline $\begin{array}{c}\text { SHORT } \\
+ \\
\text { COT1 }\end{array}$ & $\begin{array}{c}\text { LONG } \\
+ \\
\text { MOB2A }\end{array}$ & $\begin{array}{c}\text { LONG } \\
+ \\
\text { MOB2B }\end{array}$ \\
\hline $\begin{array}{c}\text { SHORT } \\
+ \\
\text { MOB2A }\end{array}$ & $\begin{array}{c}\text { SHORT } \\
+ \\
\text { MOB2B }\end{array}$ & $\begin{array}{c}\text { LONG } \\
+ \\
\text { pGADT7 }\end{array}$ \\
\hline $\begin{array}{c}\text { SHORT } \\
+ \\
\text { PGADT7 }\end{array}$ & $\begin{array}{c}\text { PGBKT7 } \\
+ \\
\text { LONG }\end{array}$ & $\begin{array}{c}\text { PGBKT7 } \\
+ \\
\text { SHORT }\end{array}$ \\
\hline
\end{tabular}
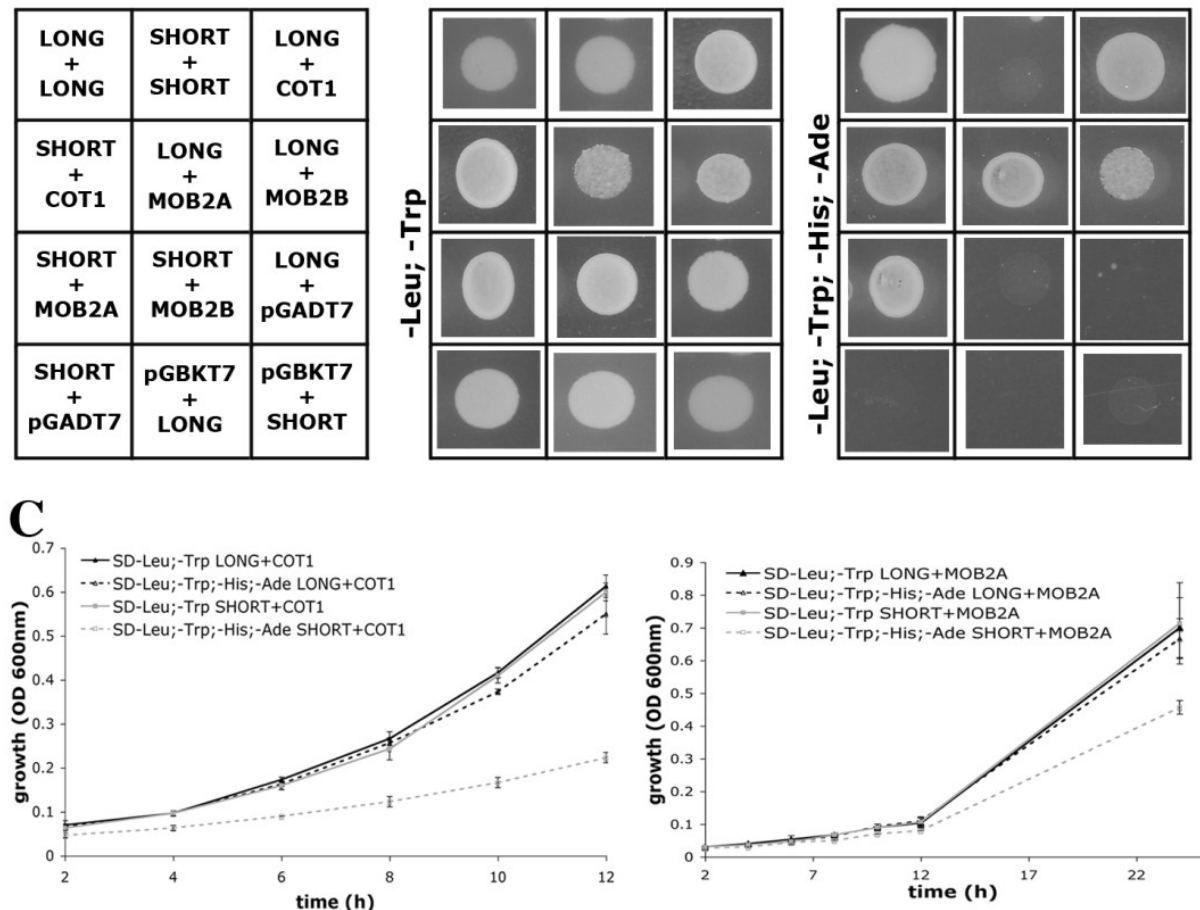

Figure III-7 Homo- and heterodimerization of COT1 requires overlapping regions. (A) Yeast two-hybrid analysis of COT1 complex components. Genes cloned into pGBKT7 and pGADT7 (mentioned as $1^{\text {st }}$ or $2^{\text {nd }}$ fusion, respectively) were co-expressed as fusions with the GAL4 DNA-binding domain and activation domains, respectively. Plasmids expressing the indicated proteins either as prey or bait alone were used as negative controls. pGBKT7-53 (murine p53) and pGADT7-recT (SV40 large T antigene) fusions were used as positive control. (B) Interaction analysis of two N-terminal COT1 fragments. (C) Growth curves of yeast harboring the indicated two hybrid plasmids in liquid medium. 


\section{Discussion}

As part of our comparative characterization we have assigned distinct cellular functions to the four members of the MOB family. The two NDR kinases present in $N$. crassa interact with a specific subset of MOB adaptors. COT1 is regulated by the combined function of MOB2A and MOB2B. This is indicated by the copurification of COT1 with these two MOBs, the cot-like synthetic phenotype of the $\Delta m o b-2 a ; \Delta m o b-2 b$ double mutant and the deletion's impact on the in vitro kinase activity of COT1. Based on our findings that include the reduction in kinase activity in mob mutants and synthetic interaction of mob mutants with cot-1(ts), we believe that MOB2A contributes more to COT1 function than MOB2B. Currently, we do not understand, why two close paralogs have evolved in $N$. crassa. We have, however, indications that several splice variants of MOB2 proteins are expressed in N. crassa (Maerz and Seiler, unpublished), which may suggest additional levels of COT1 regulation through the two MOB2 proteins.

The cot-1 locus allows the translation of two isoforms. The length of the N-terminus of the short isoform corresponds to the $\mathrm{N}$-terminus of animal NDR kinases, while the long version contains a fungal-specific extension of 118 amino acids. We show that the region directly preceding the kinase domain (amino acids 119-212 of COT1(long)) is sufficient for homodimerization of COT1. Furthermore, this region is also responsible for the interaction of COT1 with MOB2 proteins, suggesting the presence of either COT1 homoor COT1-MOB2 heterodimers in the cell and a potential for regulating NDR function at the level of dimmer formation. The sequence of the $\mathrm{N}$-terminal extension is poorly conserved, but its presence is a feature of most fungal NDR kinases and characterized by a high abundance of the amino acids asparagine and glutamine (e.g. 24\% and 36\% asparagine and glutamine residues in $N$. crassa COT1 and S. cerevisiae Cbk1p, respectively). This region is required for stabilization of kinase homo- and of COT1-MOB2 heterodimers and is also involved in the stimulation of the kinase activity. The fact that the level of MOB binding to COT1(long) and COT1(short) in vivo is similar, yet their in vitro kinase activities are different, indicates that kinase activation is not a mere consequence of $\mathrm{MOB}$ binding, but requires additional level of regulation. This may include additional interacting proteins and/or posttranslational modifications of the kinase. 
The reduced stability of COT1(short) homodimers may be a reason for the degradation of COT1(short) in the mob-2 double deletion background. This prediction is consistent with data from budding and fission yeast. The size of ORB6 corresponds to the short COT1 isoform, and shutoff experiments of mob-2 in S. pombe result in degradation of ORB6. Budding yeast Cbk1p, however, has a long $\mathrm{N}$-terminal extension and deletion of mob-2 does not affect Cbk1p stability in this yeast (Hergovich et al., 2006; Jansen et al., 2006). The presence of a second ATG and thus the potential for the presence two expressed isoforms in N. crassa and related species (e.g. Podospora anserina, Magnaporthe grisea and Sclerotinia sclerotiorum) allows the prediction that the stability of animal NDR kinases is also regulated through their interaction with MOB proteins. However, no data are currently available regarding animal knock down experiments of MOB proteins and their impact on endogenous NDR levels.

We compared the expression profiles of the four mob and the two $n d r$ kinase genes within a growing colony (whole genome microarray data are available at http://bioinfo.townsend.yale.edu/index.jsp; Kasuga and Glass, 2008). These data indicate that cot-1 and mob-2a were expressed at higher levels in the peripheral region of the colony, while $d b f-2$ displayed equal expression levels in young and older regions of the colony. mob-1, mob-2b and mob-3 RNAs were not even detected in the youngest section, but constant throughout the remaining colony (Supplementary Figure S III-2 A). These expression profiles are consistent with the observed defects in the respective mutants, and support functions of COT1 and MOB2A during tip growth, while the remaining proteins may primarily function in subapical regions.

We have previously shown that the mRNA levels of the two COT1 transcripts are photoregulated. In dark-grown cultures the large transcript is favored, while light induced the expression of the small transcript (Lauter et al., 1998). To extend this analysis, we used our endogenously tagged myc-cot-1 strain and assayed for COT1 expression in cells are grown under vegetative conditions either in the light or dark as well as under conditions favoring asexual or sexual development. COT1 protein level decreased during the sexual development, but COT1 abundance was constant in the other conditions (supplementary Figure III-2 B). More importantly, the ratio of the two isoforms did not change significantly during any growth condition. Thus, the light-dependent differences in mRNA abundance 
do not translate into significantly different protein levels of the two COT1 isoforms, and the significance of these mRNA profiles is currently unclear.

The mutant characteristics and our coprecipitation data indicate that the primary adaptor of the NDR kinase DBF2 is MOB1. This NDR-MOB pair is important for connecting mitotic exit with septum formation in both yeasts and in Aspergillus nidulans (Kim et al., 2006; Krapp and Simanis, 2008), and mutations in components of the septation initiation network (SIN) result in aseptate strains. A full set of SIN components can be detected in the genome of N. crassa (e.g. CDC7/NCU01335; SPG1/NCU08878; CDC14/NCU06636; SID1/NCU04096; CDC11/NCU03545; SID2/NCU09071; MOB1/NCU01605), suggesting that this network operates in a similar manner in N. crassa. This is further supported by the fact that all mutants in these components display defects in septum formation (Maerz and Seiler, unpublished). However, in contrast to unicellular fungi (Krapp et al., 2004; Luca and Winey, 1998; Salimova et al., 2000; Schweitzer and Philippsen, 1991), SIN components are not essential for vegetative viability in N. crassa, and mutants still allow filamentous growth and colony formation. However, conidiation, which resembles a budding-type growth program and also requires septum formation, is abolished in $\Delta m o b$ 1 and $\Delta d b f-2$ and only regained in strains harboring suppressor mutations. In addition to their function in septation, our data also indicate a function of the two SIN components during sexual development. The fact that we were unable to isolate viable haploid progeny harboring only $\Delta d b f-2$, along with the presence of viable hygromycin resistant $d b f-2^{+}$progeny and the abnormal shape and size of the generated ascospores, suggests that DBF2 may have an essential function during meiosis. In contrast to $d b f-2$, the deletion of mob-1 also impaired meiosis, but functional ascosporogenesis was still possible, albeit at a reduced rate. A possible function of MOB1/DBF2 in controlling the mitotic cell cycle and coordinating mitotic exit with septation was not analyzed here, but may be suggested by the cognate yeast and $A$. nidulans mutants.

It was interesting that double mutants of conditional cot-1(ts) or pod-6(ts) strains with $\Delta m o b-1$ displayed synthetic defects that were more prominent than the growth defects observed in mutant combinations with $\Delta m o b-2 b$. This suggests that the deletion of $m o b-1$ has more impact on the function of COT1 than the absence of the direct COT1-interactor MOB2B. Indirect data from budding yeast suggest an involvement of MEN function in regulating the localization of Mob2p and Cbk1p (Weiss et al., 2002). In addition, the 
activity of the SIN components CDC7 and SID1 has been shown to control ORB6 activity in interphase fission yeast cells (Kanai et al., 2005; Weiss et al., 2002). Thus we speculate that a similar, currently poorly-understood connection between the two NDR kinase pathways also exists in $N$. crassa that may involve the presence of MOB1-bound DBF2 activity. A function of COT1 and POD6 as potential SIN effectors during septation is further supported by the localization of both proteins at the forming septum (Seiler et al., 2006).

MOB3 is required for vegetative cell-cell fusion and during sexual development. Based on these defects and the lack of synthetic interactions, it has a function unrelated to NDR signaling and the other $\mathrm{MOB}$ proteins. The mammalian homolog phocein has been shown to interact with the multidomain protein striatin, which has been suggested as a scaffolding protein linking cell signaling and endocytosis (Benoist et al., 2006). A functional conservation of mammalian and fungal striatin genes has been demonstrated in Sordaria macrospora by complementation of the striatin/pro11 mutant with a mouse striatin cDNA (Poggeler and Kuck, 2006). Interesting is that S. macrospora pro11 and N. crassa $\Delta$ mob-3 (and also the $N$. crassa striatin deletion strain; Maerz and Seiler, unpublished) display highly similar developmental defects resulting in arrested protoperithecial development and subsequently sterility. This suggests the presence of a conserved signaling complex required for developmental decisions in filamentous ascomycetes and higher eukaryotes, yet not in unicellular yeasts, which lack detectable mob-3 and striatin genes. 


\section{Experimental procedures}

\section{Strains, media and growth conditions}

Strains used in this study are listed in Table III-2 (see also McCluskey, 2003). General genetic procedures and media used in the handling of $N$. crassa have been described (Davis, 2000; Davis and DeSerres, 1970) or are available through the Fungal Genetic Stock Center (www.fgsc.net). Microscopic documentation of fungal hyphae or colonies was performed with an SZX16 stereomicroscope, equipped with a Colorview III camera and Cell ${ }^{\mathrm{D}}$ imaging software (Olympus, Japan) or an ORCA ER digital camera (Hamamatsu, Japan) mounted on an Axiovert S100 microscope (Zeiss, Germany). Image acquisition was done using the Openlab 5.01 software (Improvision, Great Britain) and images were further processed using Photoshop CS2 (Adobe, USA).

Table III-2 Neurospora crassa strains used in this study

\begin{tabular}{|c|c|c|}
\hline Strain & Genotype & Source \\
\hline wild type & 74-OR23-1 Mat A & FGSC \#987 \\
\hline wild type & ORS-SL6 Mat a & FGSC \#4200 \\
\hline $\cot -1(t s)$ & $\cot -1(C 102 t)$ & FGSC \#4066 \\
\hline pod-6(ts) & pod-6(I310K) & Seiler et al., 2006 \\
\hline$\Delta$ mob-1 (heterokaryon) & 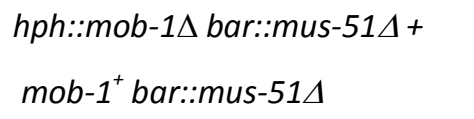 & FGSC \#11487 \\
\hline$\Delta m o b-1$ & $h p h:: m o b-1 \Delta$ & FGSC \#11487 x FGSC \#987 \\
\hline$\Delta m o b-2 a$ & $h p h:: m o b-2 a \Delta$ & FGSC \#11296 \\
\hline$\Delta m o b-2 b$ & $h p h:: m o b-2 b \Delta$ & FGSC \#13575 \\
\hline$\Delta m o b-3$ & 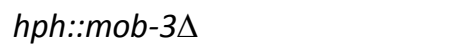 & FGSC \#12362 \\
\hline$\Delta d b f-2$ & 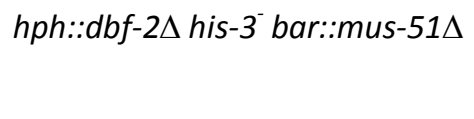 & $\begin{array}{l}\text { microconidia of FGSC } \\
\# 12000\end{array}$ \\
\hline$\Delta m o b-1 ; \cot -1(t s)$ & $h p h:: m o b-1 \Delta \cot -1(C 102 t)$ & this study \\
\hline$\Delta m o b-2 a ; \cot -1$ (ts) & $h p h:: m o b-2 a \Delta$ cot-1(C102t) & this study \\
\hline$\Delta m o b-2 b ; \cot -1(t s)$ & $h p h:: m o b-2 b \Delta \cot -1(C 102 t)$ & this study \\
\hline$\Delta m o b-3, c o t-1(t s)$ & $h p h:: m o b-3 \Delta \cot -1(C 102 t)$ & this study \\
\hline$\Delta m o b-1 ; p o d-6(t s)$ & hph::mob-1د pod-6(I310K) & this study \\
\hline$\Delta m o b-2 a ; p o d-6(t s)$ & hph::mob-2as pod-6(I310K) & this study \\
\hline
\end{tabular}




\begin{tabular}{|c|c|c|}
\hline Strain & Genotype & Source \\
\hline wild type & 74-OR23-1 Mat A & FGSC \#987 \\
\hline wild type & ORS-SL6 Mat a & FGSC \#4200 \\
\hline $\cot -1(t s)$ & $\cot -1(C 102 t)$ & FGSC \#4066 \\
\hline pod-6(ts) & pod-6(I310K) & Seiler et al., 2006 \\
\hline$\Delta m o b-1$ (heterokaryon) & 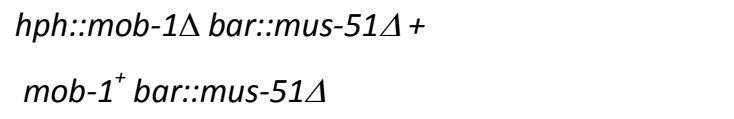 & FGSC \#11487 \\
\hline$\Delta m o b-1$ & 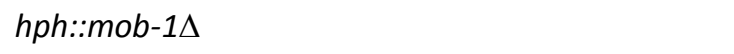 & FGSC \#11487 x FGSC \#987 \\
\hline$\Delta m o b-2 a$ & $h p h:: m o b-2 a \Delta$ & FGSC \#11296 \\
\hline$\Delta m o b-2 b$ & $h p h:: m o b-2 b \Delta$ & FGSC \#13575 \\
\hline$\Delta m o b-3$ & $h p h:: m o b-3 \Delta$ & FGSC \#12362 \\
\hline$\Delta d b f-2$ & 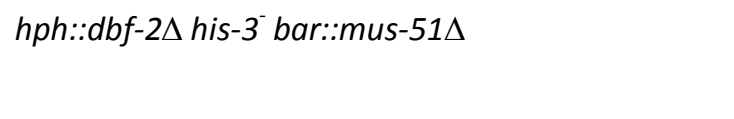 & $\begin{array}{l}\text { microconidia of FGSC } \\
\# 12000\end{array}$ \\
\hline$\Delta m o b-1 ; \cot -1$ (ts) & $h p h:: m o b-1 \Delta$ cot-1(C102t) & this study \\
\hline$\Delta m o b-2 a ; \cot -1(t s)$ & $h p h:: m o b-2 a \Delta$ cot-1(C102t) & this study \\
\hline$\Delta m o b-2 b ; \cot -1(t s)$ & $h p h:: m o b-2 b \Delta \cot -1(c 102 t)$ & this study \\
\hline$\Delta m o b-3, c o t-1(t s)$ & $h p h:: m o b-3 \Delta \cot -1(C 102 t)$ & this study \\
\hline$\Delta m o b-1 ; p o d-6(t s)$ & hph::mob-1s pod-6(I310K) & this study \\
\hline$\Delta m o b-2 a ; p o d-6(t s)$ & hph::mob-2as pod-6(I310K) & this study \\
\hline$\Delta m o b-2 b ; p o d-6(t s)$ & hph ::mob-2bs pod-6(I310K) & this study \\
\hline$\Delta m o b-3 ; p o d-6(t s)$ & hph::mob-3s pod-6(I310K) & this study \\
\hline myc-cot-1(long) & cot-1(C102t) his-3::pqa2-myc5-his6-cot-1(1-1842) & this study \\
\hline myc-cot-1(short) & $\begin{array}{l}\text { cot-1(C102t) his-3::pqa2-myc5-his6-cot-1(433- } \\
\text { 1842) }\end{array}$ & this study \\
\hline myc-cot-1 & myc-cot-1(183-4) & this study \\
\hline$m y c-c o t-1 ; \Delta m o b-2 a$ & hph::mob-2as myc-cot-1(183-4) & this study \\
\hline$m y c-c o t-1 ; \Delta m o b-2 b$ & hph::mob-2bs myc-cot-1(183-4) & this study \\
\hline$\Delta m o b-2 a ; \Delta m o b-2 b$ & $h p h:: m o b-2 a \Delta h p h:: m o b-2 b \Delta$ & this study \\
\hline $\begin{array}{l}\Delta m o b-2 a ; \Delta m o b-2 b ; m y c- \\
\text { cot-1 }\end{array}$ & 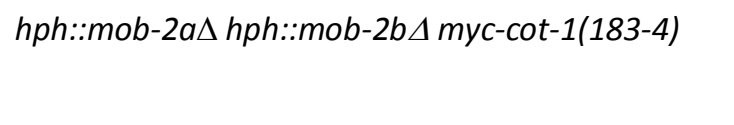 & this study \\
\hline trp-1;his-3 & $\operatorname{trp}-1^{-}$his-3 & FGSC\#4050 x FGSC\#6103 \\
\hline myc-cot-1;his-3 & myc-cot-1(183-4) his-3 & this study \\
\hline$m y c-d b f 2(E C)$ & PgpdA-myc-his-dbf2::nat(EC) & this study \\
\hline$m y c-d b f 2(E C) ; h i s-3$ & PgpdA-myc-his-dbf2::nat(EC);his-3- & this study \\
\hline$H A-m o b-1$ & his-3::Pccg-1-HA-mob-1;nic-3 & this study \\
\hline$H A-m o b-2 a$ & his-3::Pccg-1-HA-mob-2a;nic-3 & this study \\
\hline myc-cot-1(D337A) & myc-cot-1(D337A) & this study \\
\hline
\end{tabular}




\section{Tagged constructs}

The myc-tagged cot-1 replacement cassette, pCZ18 (Seiler et al., 2006) was linearized with Xhol and co-transformed with a HindIII/BamHI genomic fragment containing the his3 gene in cot-1; $\Delta$ mus-51; his-3 strain. Transformants where screened for their ability to grow on minimal medium at $34^{\circ} \mathrm{C}$. Five transformants were backcrossed to obtain the tagged cot-1 allele in a wild type background. Proper integration of the myc-cot-1 cassette at the cot-1 locus (and the replacement of the native cot-1 ORF) was verified by Southern blot analysis and by sequencing. To generate myc-cot-1(D337A) pCZ18 was mutagenized using the QuikChange site-directed mutagenesis kit (Stratagene, La Jolla, CA) according to the manufacturer's protocol using the oligonucleotides CAC GGT TGT GCA TGC AGA GCT ATT AAG CCA GAC and its complement (not specified) for the mutagenesis.

For the expression of double-tagged cot-1 constructs, the pQA2-myc-his vector was used, that allowed the expression of $\mathrm{N}$-terminally 3xmyc-6xhis-tagged proteins from the his-3 locus (He et al., 2005a). PCR fragments containing genomic DNA of the long or short cot-1 ORF, respectively, and their 3'-UTRs were cloned into pQA-myc-his. The primers used for myc-his-COT1(long) were AGA CAA GGT GAA TTC ATG GAC AAC and AGC AAG CGC TAG TTG TAT TT, and for myc-his-COT1(short) TAT CTG AGC GAA TTC ATG CCT TCG and CGT ATC CCG GGC ATA GTA TT. The resulting constructs were transformed by electroporation into a cot-1(ts); his-3 strain at the his-3 locus.

To generate tagged $d b f-2$ (NCU9071), the 3xmyc-6xhis tag was excised using Dral and Cfrgl from the pQA-myc-his plasmid and blunted using Klenow DNA polymerase. The obtained fragment was cloned between the Pgpd promoter and TrpC terminator of pEHN1nat (kindly provided by Stefanie Pöggeler), which was linearized with $\mathrm{Ncol}$ and blunted with Mung Bean nuclease. PCR-amplified fragments of the genomic $d b f-2$ ORF were obtained using the primers GGC GCG CCT ATG TCT AGC TAC TTG ACA AAC TTC and CTA GGA TCC CTA CAG CAT CGT ACC AAA ATT G. The integrity of the myc-his-dbf-2 expression vector was verified by sequencing prior to its transformation into wild type or his-3 protoplasts. Transformants were selected on minimal medium containing $30 \mu \mathrm{g} / \mathrm{ml}$ nourseothricin.

PCR-amplified fragments of the respective mob ORF were cloned into pHAN1 (Kawabata and Inoue, 2007), allowing expression of HA-tagged proteins from the his-3 locus. The following primers were used: GGG ATC CAT GAG CTC CTT TCT TAC GAC C and GGG GCC 
cCCT AGT CGC TGC GTA ACA TGC for $m o b-1$ and GCC CGG GTA TGG ATC CCA ATA ATG GTT CG and GGA ATT CCT AGC TCG AGG GTG GGC C for mob-2a. For expression of HA- tagged MOB proteins, the HA constructs were transformed into nic-3; his-3 or trp-1;his-3 protoplasts, respectively, and were selected for complementation of the his-3 auxotrophy. Immunoprecipitation was performed with cell extracts from fused, heterokaryotic, strains that were selected by their ability to grow on minimal media lacking supplements.

\section{Yeast Two-Hybrid Assays}

The Matchmaker Two-Hybrid system 3 (Clontech, USA) was used according to the manufacturer's instructions. cDNA of the indicated genes was amplified with primers spanning the ORFs from start to stop codons as annotated by the $N$. crassa database (http://www.broad.mit.edu/annotation/fungi/neurospora_crassa_7/index.html) and cloned either into the pGADT7 vector containing the GAL4 activation domain or into pGBKT7 containing the DNA-binding domain using the following primers: MOB1EcoRI-5' (GGA ATT CAT GAG CTC CTT TCT TAC GAC C), MOB1BamHI-3' (CG G GT CCC TAG TCG CTG CGT AAC ATG C), MOB2aEcoRI-5' (GGA ATT CAT GTC CAA CCT CTT TTC TGG AA), MOB2aEcoRI-3' (GGA ATT CCT AGC TCG AGG GTG GGC C), MOB2bEcoRI-5' (GGA ATT CAT GTC TTG GAG CTC AGC CAA C), MOB2bBamHI-3' (CGG GAT CCT TAA GCC AGG CCT GCC ATC TG), COT1longEcoRI-5' (GGA ATT CAT GGA CAA CAC CAA CCG CC), COT1BamHI-3' (CGG GAT CCT TAT CGG AAG TTG TTG TCG AAA C), COT1shortEcoRI-5' (CGG AAT TCA TGC CTT CGA ATA CCC AGA CC), COT1-N-termBamHI-3' (CGG GAT CCT TAC TCG GGC TTG TCC TTG GTT C), POD6Ndel-5' (GAT CAG CAT ATG GCG ACC CTA TCG GTA TAC), POD6EcoRI-3' (GGA ATT CCT ACC TCC CTC AGA CAC TCG TG). The fusion proteins were (co-)expressed in S. cerevisiae $\mathrm{AH} 109$ and potential interactions determined by activation of lacZ or his 3 and ade 2 reporter constructs that discriminate positive interactions on the basis of color in the presence of X- $\alpha$-galactopyranoside or by selection for viability on SD medium lacking adenine and histidine. 


\section{NDR kinase purification and identification of copurifying proteins}

All buffers contained the following phosphatase and protease inhibitors: $20 \mathrm{mM} \beta$ glycerophosphate, $2 \mathrm{mM} \mathrm{Na}_{3} \mathrm{VO}_{4}, 5 \mathrm{mM} \mathrm{NaF}, 0.5 \mathrm{mM}$ PMSF, $1 \mathrm{mM}$ benzamidine, $1 \mathrm{mM}$ DTT, $1 \mu \mathrm{g} / \mathrm{ml}$ pepstatin A, $5 \mu \mathrm{g} / \mathrm{ml}$ aprotinin. Mycelial samples were frozen in liquid nitrogen, pulverized and suspended either in lysis buffer 1 for immunoprecipitation experiments ( $20 \mathrm{mM}$ Tris pH 7.5, $150 \mathrm{mM} \mathrm{NaCl}, 10 \%$ glycerol, $0.5 \mathrm{mM}$ EDTA, 0.1\% NP-40,) or in lysis buffer 2 for double tag his-myc purifications $(20 \mathrm{mM}$ Tris $\mathrm{pH} 7.5,150 \mathrm{mM} \mathrm{NaCl}$, $10 \%$ glycerol, $25 \mathrm{mM}$ glucose, $0.01 \%$ triton $\mathrm{X}-100)$. For the double tag purification, $25 \mathrm{ml}$ cleared crude extract (10 min, $16.000 \mathrm{~g}$ ) were incubated for $2 \mathrm{~h}$ at $4^{\circ} \mathrm{C}$ with $500 \mu \mathrm{l} \mathrm{Ni-NTA}$ agarose beads (Quiagen, Germany). The beads were washed twice with washing buffer (20 mM Tris pH 7.5, $500 \mathrm{mM} \mathrm{NaCl}, 10 \%$ glycerol, $25 \mathrm{mM}$ glucose, $0.01 \%$ triton $\mathrm{X}-100$ ) and were eluted in $20 \mathrm{mM}$ Tris pH 7.5, $137 \mathrm{mM} \mathrm{NaCl}, 10 \%$ glycerol, $25 \mathrm{mM}$ glucose, $0.01 \%$ triton X-100, 200mM imidazole. For the immunoprecipitation, the supernatant of cell extract (10 $\mathrm{min} ; 16.000 \mathrm{~g}$ ) or the elution fraction of the Ni-NTA purification were incubated with monoclonal 9E10 anti-myc (Santa Cruz, USA) antibody on a rotation device for $2 \mathrm{~h}$ at $4^{\circ} \mathrm{C}$, and with Protein-A-Sepharose beads for additional $1 \mathrm{~h}$ at $4^{\circ} \mathrm{C}$. The beads were washed three times ( $20 \mathrm{mM}$ Tris $\mathrm{pH} 7.5,500 \mathrm{mM} \mathrm{NaCl}, 10 \%$ glycerol, $0.5 \mathrm{mM}$ EDTA, $0.1 \%$ NP-40). Immunoprecipitated proteins were recovered by boiling the beads for 10 min at $98^{\circ} \mathrm{C}$ in Laemmli buffer and separated by $10 \%$ SDS-PAGE. Quantification of precipitated proteins were performed by densiometry using the software AIDA Image Analyzer 4.2.2 (Raytest, USA) of Coomassie Brillant Blue stained gels (Blum et al., 1987).

For protein identification by LC-MS, peptides of the in-gel trypsinated proteins (Shevchenko et al., 1996) were extracted from gel slices of silver stained protein bands and separated on a Dionex NAN75-15-03-C18 PM column with an ultimate3000 HPLC system (Dionex, Amsterdam, Netherlands) prior to mass analyses with an LCQ DecaXP mass spectrometer (Thermo Electron Corp., San Jose, USA). Cycles of MS spectra with $\mathrm{m} / \mathrm{z}$ ratios of peptides and four data-dependent MS2 spectra were recorded by mass spectrometry. The "peak list" was created with extractms provided by the Xcalibur software package (BioworksBrowser 3.3.1SP1). The MS2 spectra were analyzed against the $N$. crassa genome protein database using the TurboSEQUEST program (Eng et al., 1994) of the Bioworks software (Version 3.1, Thermo Electron; Germany). Protein identification required at least two different high scoring peptides meeting the following 
criteria: i) XCorr $(1+, 2+, 3+)>2.0,2.5,3.0$. ii) $\Delta C n>0.4$. iii) $\mathrm{Sp}>500$. MS2 spectra of the highest scoring peptides were manually verified.

\section{COT1 activity assays}

Mycelial samples were frozen in liquid nitrogen, pulverized and resuspended in immunoprecipitation (IP) buffer (50 mM Tris $\mathrm{pH} 7.5,100 \mathrm{mM} \mathrm{KCl}, 10 \mathrm{mM} \mathrm{MgCl}, 0.1 \%$ NP40, $20 \mathrm{mM} \beta$-glycerophosphate, $2 \mathrm{mM} \mathrm{Na}_{3} \mathrm{VO}_{4}, 5 \mathrm{mM} \mathrm{NaF}, 0.5 \mathrm{mM}$ PMSF, $1 \mathrm{mM}$ benzamidine, $2 \mathrm{mM}$ EGTA, $1 \mathrm{mM}$ DTT, $1 \mu \mathrm{g} / \mathrm{ml}$ pepstatin A, $5 \mu \mathrm{g} / \mathrm{ml}$ aprotinin). The samples were homogenized, centrifuged at $4.000 \mathrm{~g}$ for $15 \mathrm{~min}$, and the supernatant subjected to a second centrifugation step for $15 \mathrm{~min}$ at $22.000 \mathrm{~g}$. To equalize the protein concentration of the crude extracts protein content was measured by a Bradford assay using Roti-Quant (ROTH, Germany). For immunoprecipitation, $1 \mathrm{ml}$ aliquots of crude extracts were incubated for $2 \mathrm{~h}$ at $4^{\circ} \mathrm{C}$ on a rotation device with $1.5 \mu \mathrm{g}$ anti-myc antibody 9E10 (Santa Cruz, USA). The antigen-antibody complexes were recovered using protein Asepharose (Amersham, UK) and washed once with IP buffer, twice with IP buffer containing $1 \mathrm{M} \mathrm{NaCl}$ followed by two times with kinase reaction buffer $(20 \mathrm{mM}$ Tris $\mathrm{pH}$ 7.5, $10 \mathrm{mM} \mathrm{MgCl}$, $1 \mathrm{mM}$ DTT, $1 \mathrm{mM}$ benzamidine, $1 \mathrm{mM} \mathrm{Na}_{3} \mathrm{VO}_{4}, 5 \mathrm{mM} \mathrm{NaF}$ ). Kinase assays were performed using a modified protocol described previously for $\mathrm{Ndr}$ kinase (Millward et al., 1998). Briefly, beads were resuspended in $30 \mu$ l kinase reaction buffer containing $2 \mathrm{mM}$ of synthetic substrate peptide (KKRNRRLSVA), $0.5 \mathrm{mM}$ ATP and $1 \mu \mathrm{Ci}$ $\left.{ }^{32} \mathrm{P}\right]$ ATP. After incubation for $1 \mathrm{~h}$ at $37^{\circ} \mathrm{C}$, samples were centrifuged for $5 \mathrm{~min}$ at $16.000 \mathrm{~g}$, the supernatant was spotted onto P81 phosphocellulose paper circles (Whatman, UK). Dried circles were washed 5 times for 30 min with 1\% phosphoric acid and once with acetone before incorporation of phosphate into the substrate peptide was measured by liquid scintillation counting. The remaining protein-sepharose pellet was boiled for $10 \mathrm{~min}$ in Laemmli buffer and the supernatant was used to determine the myc-COT1 concentration in the kinase reaction by SDS-PAGE and Western blot. 


\section{Acknowledgment}

This research project was financially supported by the Deutsche Forschungsgemeinschaft through the DFG Research Center of Molecular Physiology of the Brain (SS), The Israel Science Foundation (OY) and by a joint DFG research grant to SS and OY (SE 1054/3-1). We thank Chen She (Beijing Normal University, China) for his technical assistance during the early stage of this project. We thank Takao Kasuga (University of California, Davis, USA) for providing the mRNA profiling dataset and for his help with the analysis. 


\section{Supplementary Data}

\section{fungal specific N-terminal extension}

s.c. Cbk1p

U.m. U404956

A.n. $\cot A$

c. $t$. COI 1

c.p. CPCOT1

M.g. HQG 05376

N. G. COI1 (long)

N. a. COIl (short)

N. .P. ORB6

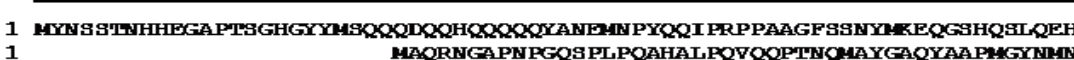

S.c. Cbk1p
U.m. UN04956
A.n. CotA
C.t. COT1
C.P. CPCOT1
M.g. HOS 05376
N.c. COI1 (long)
N.c. COI1 (short)
S.P. ORB6
H.s. NDR2

\section{fungal specilic N-terminal extension}

71 LQRETENLGSGFTDW PALWYPATPPFHNNYAASWQUINTPPPIEGGLYRHNNNSQGMVQNGNGSGNAQLP 47 L.ASNHGPVAYGFSAAGQGGFAQS - QAYAQQASAAHQYHAPPHLHAQIHQAAIAAQAQAPPSQLQHPAMSQ 10 INFGYNRRAFNPAAANNRAYPTTPSAFPQPIYQSQSPCDYMIMAQNG . . . . . . . . . . . VYGQGYF

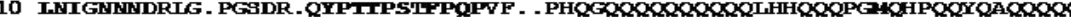

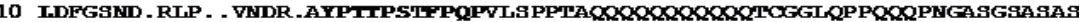

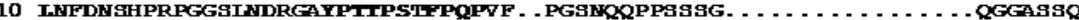
9 I.NLGINIDTRAM. P .NDR .TYPTTPSTFPQPVF. . PGQ . . . . . . . . . . . . . . . . . QAGGSQQ

\section{S.c. Cbk1p \\ U.m. Uw04956 \\ A.n. Cota \\ c.t. COI 1 \\ C.P. CPCOT1 \\ M.g. MGG 05376 \\ N. G. COI1 (long) \\ N. c. COM1 (short) \\ S.P. ORB6}

\section{fungal specilic N-terminal extension}

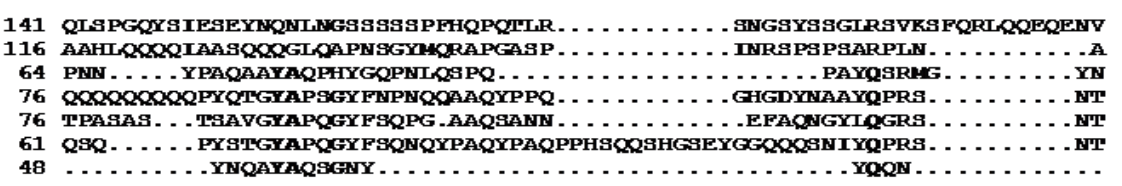

48 Q. . . PYSTGYAPQGYFSQNQYPAQYPAQPPHSQQSHGSEYGGQQQSNIYQPRS $\ldots \ldots \ldots \ldots$ s.c. Cbk1p

V.m. 0404956

A.n. Cota

c.t. COT1

C.P. CRCOT1

M.g. HGG 05376

N. G. COI1 (long)

N. $a$. COI1 (short)

S.P. ORB6

H.S. NDR2

\begin{tabular}{|c|c|c|}
\hline & fungal specific N-terminal extension & \\
\hline 199 & 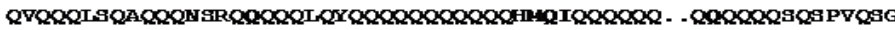 & BFNNG . . . . TI \\
\hline 163 & 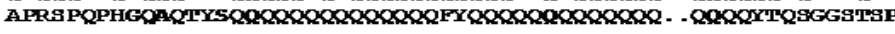 & PNPAN . . . . EK \\
\hline 98 & 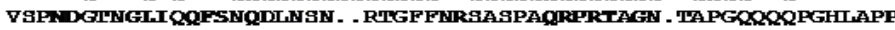 & PVPRSP \\
\hline 124 & PGIVWPNVGIAHQFSHQNLGGAARASPYGSRGPSPGQRPRTAEA. SGQPPSGYGHATPE & PLPNQR \\
\hline 119 & PGIVDPNVGLAHQFSHQNLGGSVRSAP . . .RGPPSSQRPRTAGSQPAGAYAGYSNSNAPF & PLPTQSGISTV \\
\hline 115 & PGINDPNVGIAHQFSQQNLR. . . . APYGSRGPSPAGRPRTACA. PGQRP . . YGGYHSQI & MAPPAPAQYPA \\
\hline 63 & 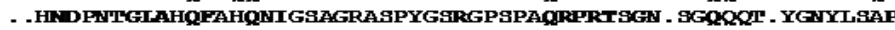 & PAPSN . . . . TQ \\
\hline 1 & & MPSN . . . . TQ \\
\hline 1 & & MTR ........ \\
\hline
\end{tabular}

homo-fheterod inerization

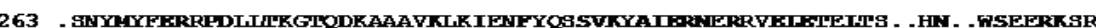

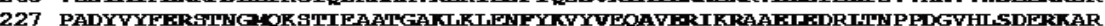

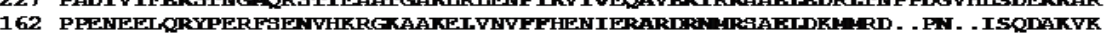

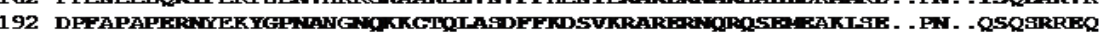

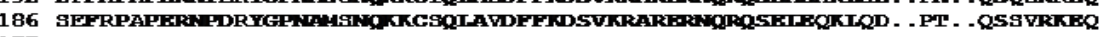

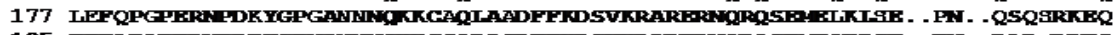

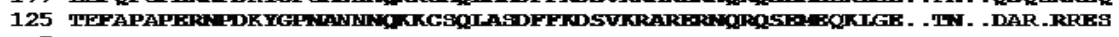

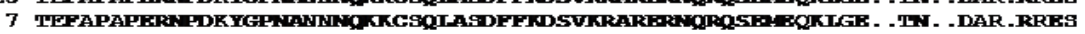

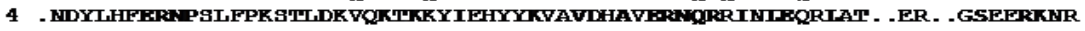

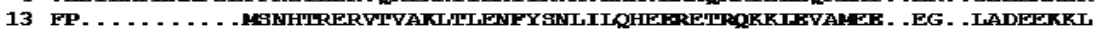

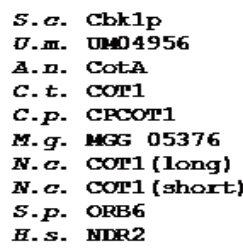

S.c. Cbk1p

A.n. $\operatorname{Cot} A$

c.t. $\operatorname{COT1}$

M.g. HOG 05376

N. c. Corl (short)

H.S. NDR2

s.c. Cbk1p

S.P. ORB6

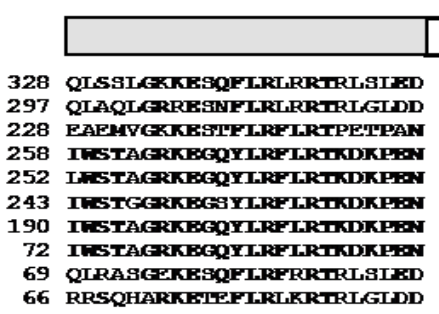

Figure S III-1 Alignment of the N-terminus of fungal NDR kinases. Sequences of functionally characterized fungal NDR kinases were aligned using Clustal W. Human NDR2 was included as an animal reference protein. S.c. Saccharomyces cerevisiae, U.m. Ustilago maydis, A.n. Aspergillus nidulans, C.t. Colletotrichum trifolii, C.p. Claviceps purpurea, M.g. Magnaporthe grisea, N.c. Neurospora crassa, S.p. Schizosaccharomyces pombe, H.s. Homo sapiens. 
A

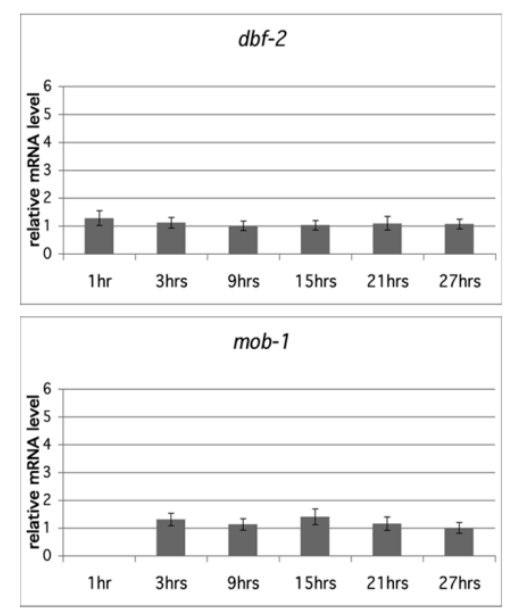

B
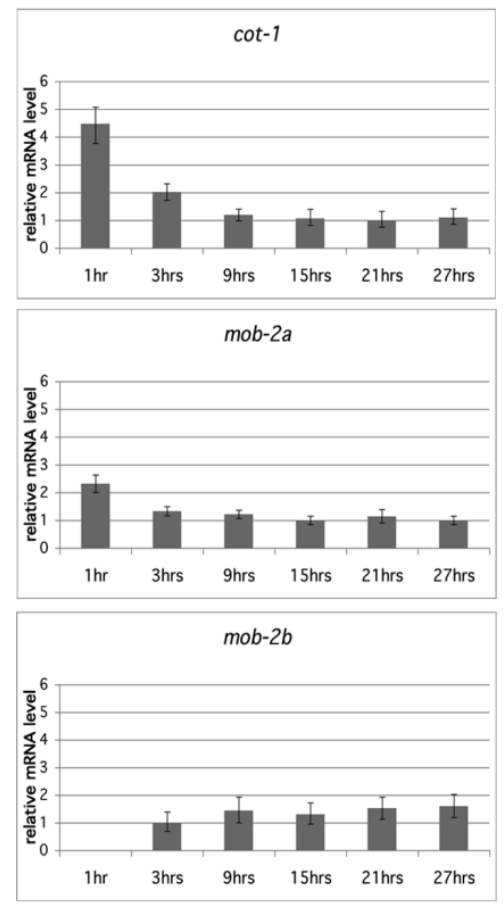

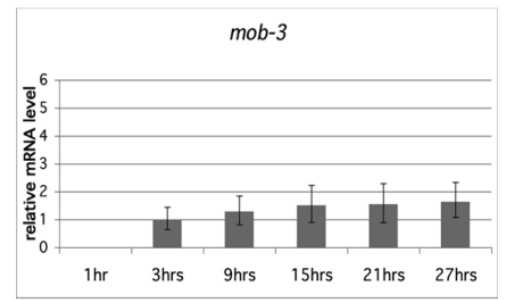

$1 \mathrm{hr} \quad 3$ hrs 9 hrs $15 \mathrm{hrs} 21 \mathrm{hrs} 27 \mathrm{hrs}$

WB: $\alpha-m y c$

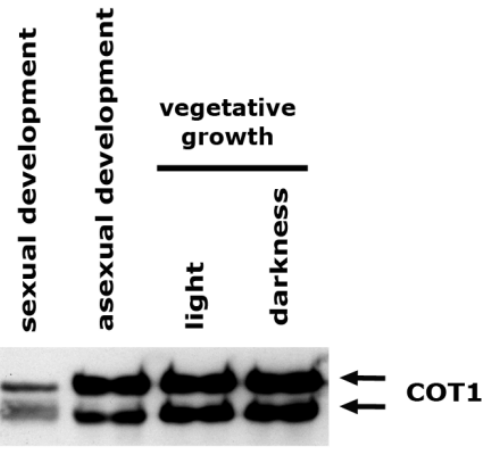

Ponceau

staining

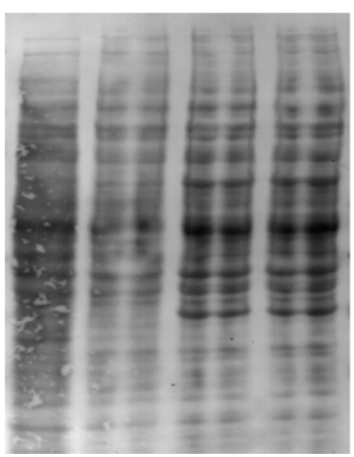

Figure S III-2 Expression profiles of the NDR kinases and MOB proteins under different growth conditions. (A) Relative mRNA distribution of the indicated genes during various stages of vegetative growth obtained by BAGEL (Bayesian Analysis of Gene Expression Levels). mRNA was isolated from discrete sections of a N. crassa colony and used for a microaray analysis. The $1 \mathrm{~h}$ time point corresponds to the most apical region. The smallest value among the entire measurement per gene is set to 1. For details see Kasuga and Glass, 2008. (B) Relative ratio of the two COT1 isoforms. Cultures for vegetative growth under constant light or darkness were grown in minimal media for $24 \mathrm{~h}$ at $37^{\circ} \mathrm{C}$. Sexual or asexual development was induced by growing cultures on corn meal agar ( 7 days) or on solid minimal media ( 4 days). Cell extracts were obtained as described in Material and Methods, normalized by a Bradford assay, and used further for immunodetection of COT1. Ponceau staining was used as a loading control. 


\section{References}

Benoist, M., Gaillard, S., and Castets, F. (2006) The striatin family: a new signaling platform in dendritic spines. J Physiol Paris 99: 146-153.

Bichsel, S.J., Tamaskovic, R., Stegert, M.R., and Hemmings, B.A. (2004) Mechanism of activation of NDR (nuclear Dbf2-related) protein kinase by the hMOB1 protein. $J$ Biol Chem 279: 35228-35235.

Blum, H., Beier, H., and Gross, H.J. (1987) Improved silver staining of plant proteins, RNA and DNA in polyacrylamide gels. Electrophoresis 8: 93-99.

Collinge, A.J., and Trinci, A.P. (1974) Hyphal tips of wild-type and spreading colonial mutants of Neurospora crassa. Arch Microbiol 99: 353-368.

Collinge, A.J., Fletcher, M.H., and Trinci, A.P.J. (1978) Physiological and cytology of septation and branching in a temperature-sensitive colonial mutant (cot-1) of Neurospora crassa. Trans. Br. Mycol. Soc. 71: 107-120.

Davis, R. (2000) Neurospora: Contributions of a Model Organism. New York: Oxford University Press.

Davis, R.D., and DeSerres, F.J. (1970) Genetic and microbiological research techniques for Neurospora crassa. Methods Enzymol. 17: 79-143.

Devroe, E., Erdjument-Bromage, H., Tempst, P., and Silver, P.A. (2004) Human Mob proteins regulate the NDR1 and NDR2 serine-threonine kinases. J Biol Chem 279: 24444-24451.

Dunlap, J.C., Borkovich, K.A., Henn, M.R., Turner, G.E., Sachs, M.S., Glass, N.L., McCluskey, K., Plamann, M., Galagan, J.E., Birren, B.W., Weiss, R.L., Townsend, J.P., Loros, J.J., Nelson, M.A., Lambreghts, R., Colot, H.V., Park, G., Collopy, P., Ringelberg, C., Crew, C., Litvinkova, L., DeCaprio, D., Hood, H.M., Curilla, S., Shi, M., Crawford, M., Koerhsen, M., Montgomery, P., Larson, L., Pearson, M., Kasuga, T., Tian, C., Basturkmen, M., Altamirano, L., and Xu, J. (2007) Enabling a community to dissect an organism: overview of the Neurospora functional genomics project. Adv Genet 57: 49-96.

Durrenberger, F., and Kronstad, J. (1999) The ukc1 gene encodes a protein kinase involved in morphogenesis, pathogenicity and pigment formation in Ustilago maydis. Mol Gen Genet 261: 281-289.

Emoto, K., He, Y., Ye, B., Grueber, W.B., Adler, P.N., Jan, L.Y., and Jan, Y.N. (2004) Control of dendritic branching and tiling by the Tricornered-kinase/Furry signaling pathway in Drosophila sensory neurons. Cell 119: 245-256.

Emoto, K., Parrish, J.Z., Jan, L.Y., and Jan, Y.N. (2006) The tumour suppressor Hippo acts with the NDR kinases in dendritic tiling and maintenance. Nature 443: 210-213.

Eng, J.K., McCormack, A.L., and Yates, J.R. (1994) An approach to correlate tandem mass spectral data of peptides with amino acid sequences in a protein database. J. Amer Soc Mass Spectrom 5: 976-989. 
Fleissner, A., Simonin, A.R., and Glass, N.L. (2008) Cell fusion in the filamentous fungus, Neurospora crassa. Methods Mol Biol 475: 21-38.

Galagan, J.E., Calvo, S.E., Borkovich, K.A., Selker, E.U., Read, N.D., Jaffe, D., FitzHugh, W., Ma, L.J., Smirnov, S., Purcell, S., Rehman, B., Elkins, T., Engels, R., Wang, S., Nielsen, C.B., Butler, J., Endrizzi, M., Qui, D., lanakiev, P., Bell-Pedersen, D., Nelson, M.A., Werner-Washburne, M., Selitrennikoff, C.P., Kinsey, J.A., Braun, E.L., Zelter, A., Schulte, U., Kothe, G.O., Jedd, G., Mewes, W., Staben, C., Marcotte, E., Greenberg, D., Roy, A., Foley, K., Naylor, J., Stange-Thomann, N., Barrett, R., Gnerre, S., Kamal, M., Kamvysselis, M., Mauceli, E., Bielke, C., Rudd, S., Frishman, D., Krystofova, S., Rasmussen, C., Metzenberg, R.L., Perkins, D.D., Kroken, S., Cogoni, C., Macino, G., Catcheside, D., Li, W., Pratt, R.J., Osmani, S.A., DeSouza, C.P., Glass, L., Orbach, M.J., Berglund, J.A., Voelker, R., Yarden, O., Plamann, M., Seiler, S., Dunlap, J., Radford, A., Aramayo, R., Natvig, D.O., Alex, L.A., Mannhaupt, G., Ebbole, D.J., Freitag, M., Paulsen, I., Sachs, M.S., Lander, E.S., Nusbaum, C., and Birren, B. (2003) The genome sequence of the filamentous fungus Neurospora crassa. Nature 422: 859-868.

Galagan, J.E., Henn, M.R., Ma, L.J., Cuomo, C.A., and Birren, B. (2005) Genomics of the fungal kingdom: insights into eukaryotic biology. Genome Res 15: 1620-1631.

Gallegos, M.E., and Bargmann, C.I. (2004) Mechanosensory neurite termination and tiling depend on SAX-2 and the SAX-1 kinase. Neuron 44: 239-249.

Geng, W., He, B., Wang, M., and Adler, P.N. (2000) The tricornered gene, which is required for the integrity of epidermal cell extensions, encodes the Drosophila nuclear DBF2-related kinase. Genetics 156: 1817-1828.

Gorovits, R., Propheta, O., Kolot, M., Dombradi, V., and Yarden, O. (1999) A mutation within the catalytic domain of COT1 kinase confers changes in the presence of two COT1 isoforms and in Ser/Thr protein kinase and phosphatase activities in Neurospora crassa. Fungal Genet Biol 27: 264-274.

He, Q., Cheng, P., He, Q., and Liu, Y. (2005a) The COP9 signalosome regulates the Neurospora circadian clock by controlling the stability of the SCFFWD-1 complex. Genes Dev. 19: 1518-1531.

He, Y., Emoto, K., Fang, X., Ren, N., Tian, X., Jan, Y.N., and Adler, P.N. (2005b) Drosophila Mob family proteins interact with the related tricornered (Trc) and warts (Wts) kinases. Mol Biol Cell 16: 4139-4152.

Hergovich, A., Bichsel, S.J., and Hemmings, B.A. (2005) Human NDR kinases are rapidly activated by $\mathrm{MOB}$ proteins through recruitment to the plasma membrane and phosphorylation. Mol Cell Biol 25: 8259-8272.

Hergovich, A., Stegert, M.R., Schmitz, D., and Hemmings, B.A. (2006) NDR kinases regulate essential cell processes from yeast to humans. Nat Rev Mol Cell Biol 7: 253-264.

Jansen, J.M., Barry, M.F., Yoo, C.K., and Weiss, E.L. (2006) Phosphoregulation of Cbk1 is critical for RAM network control of transcription and morphogenesis. J Cell Biol 175: 755-766.

Justice, R.W., Zilian, O., Woods, D.F., Noll, M., and Bryant, P.J. (1995) The Drosophila tumor suppressor gene warts encodes a homolog of human myotonic dystrophy 
kinase and is required for the control of cell shape and proliferation. Genes Dev 9: 534-546.

Kanai, M., Kume, K., Miyahara, K., Sakai, K., Nakamura, K., Leonhard, K., Wiley, D.J., Verde, F., Toda, T., and Hirata, D. (2005) Fission yeast MO25 protein is localized at SPB and septum and is essential for cell morphogenesis. Embo J 24: 3012-3025.

Kasuga, T., and Glass, N.L. (2008) Dissecting colony development of Neurospora crassa using mRNA profiling and comparative genomics approaches. Eukaryot Cell 7: 1549-1564.

Kawabata, T., and Inoue, H. (2007) Detection of physical interactions by immunoprecipitation of FLAG- and HA-tagged proteins expressed at the his-3 locus in Neurospora crassa. Fungal Genetics Newsletter 54: 5-8.

Kim, J.M., Lu, L., Shao, R., Chin, J., and Liu, B. (2006) Isolation of mutations that bypass the requirement of the septation initiation network for septum formation and conidiation in Aspergillus nidulans. Genetics 173: 685-696.

Krapp, A., Gulli, M.P., and Simanis, V. (2004) SIN and the art of splitting the fission yeast cell. Curr Biol 14: R722-730.

Krapp, A., and Simanis, V. (2008) An overview of the fission yeast septation initiation network (SIN). Biochem Soc Trans 36: 411-415.

Lai, Z.C., Wei, X., Shimizu, T., Ramos, E., Rohrbaugh, M., Nikolaidis, N., Ho, L.L., and Li, Y. (2005) Control of cell proliferation and apoptosis by mob as tumor suppressor, mats. Cell 120: 675-685.

Lauter, F.R., Marchfelder, U., Russo, V.E., Yamashiro, C.T., Yatzkan, E., and Yarden, O. (1998) Photoregulation of cot-1, a kinase-encoding gene involved in hyphal growth in Neurospora crassa. Fungal Genet Biol 23: 300-310.

Luca, F.C., and Winey, M. (1998) MOB1, an essential yeast gene required for completion of mitosis and maintenance of ploidy. Mol Biol Cell 9: 29-46.

Maerz, S., Ziv, C., Vogt, N., Helmstaedt, K., Cohen, N., Gorovits, R., Yarden, O., and Seiler, S. (2008) The nuclear Dbf2-related kinase COT1 and the mitogen-activated protein kinases MAK1 and MAK2 genetically interact to regulate filamentous growth, hyphal fusion and sexual development in Neurospora crassa. Genetics 179: 13131325.

McCluskey, K. (2003) The Fungal Genetics Stock Center: from molds to molecules. Adv Appl Microbiol 52: 245-262.

McNemar, M.D., and Fonzi, W.A. (2002) Conserved serine/threonine kinase encoded by CBK1 regulates expression of several hypha-associated transcripts and genes encoding cell wall proteins in Candida albicans. J Bacteriol 184: 2058-2061.

Millward, T.A., Heizmann, C.W., Schafer, B.W., and Hemmings, B.A. (1998) Calcium regulation of $\mathrm{Ndr}$ protein kinase mediated by $\mathrm{S} 100$ calcium-binding proteins. Embo J 17: 5913-5922.

Nelson, B., Kurischko, C., Horecka, J., Mody, M., Nair, P., Pratt, L., Zougman, A., McBroom, L.D., Hughes, T.R., Boone, C., and Luca, F.C. (2003) RAM: a conserved signaling network that regulates Ace2 $p$ transcriptional activity and polarized morphogenesis. Mol Biol Cell 14: 3782-3803. 
Poggeler, S., and Kuck, U. (2006) Highly efficient generation of signal transduction knockout mutants using a fungal strain deficient in the mammalian ku70 ortholog. Gene 378: 1-10.

Salimova, E., Sohrmann, M., Fournier, N., and Simanis, V. (2000) The S. pombe orthologue of the $S$. cerevisiae mob1 gene is essential and functions in signalling the onset of septum formation. J Cell Sci 113: 1695-1704.

Scheffer, J., Ziv, C., Yarden, O., and Tudzynski, P. (2005) The COT1 homologue CPCOT1 regulates polar growth and branching and is essential for pathogenicity in Claviceps purpurea. Fungal Genet Biol 42: 107-118.

Schweitzer, B., and Philippsen, P. (1991) CDC15, an essential cell cycle gene in Saccharomyces cerevisiae, encodes a protein kinase domain. Yeast 7: 265-273.

Seiler, S., and Plamann, M. (2003) The genetic basis of cellular morphogenesis in the filamentous fungus Neurospora crassa. Mol Biol Cell 14: 4352-4364.

Seiler, S., Vogt, N., Ziv, C., Gorovits, R., and Yarden, O. (2006) The STE20/germinal center kinase POD6 interacts with the NDR kinase COT1 and is involved in polar tip extension in Neurospora crassa. Mol Biol Cell 17: 4080-4092.

Shevchenko, A., Wilm, M., Vorm, O., and Mann, M. (1996) Mass spectrometric sequencing of proteins silver-stained polyacrylamide gels. Anal Chem 68: 850-858.

Song, Y., Cheon, S.A., Lee, K.E., Lee, S.Y., Lee, B.K., Oh, D.B., Kang, H.A., and Kim, J.Y. (2008) Role of the RAM network in cell polarity and hyphal morphogenesis in Candida albicans. Mol Biol Cell 19: 5456-5477.

Stegert, M.R., Hergovich, A., Tamaskovic, R., Bichsel, S.J., and Hemmings, B.A. (2005) Regulation of NDR protein kinase by hydrophobic motif phosphorylation mediated by the mammalian Ste20-like kinase MST3. Mol Cell Biol 25: 11019-11029.

Walther, A., and Wendland, J. (2003) Septation and cytokinesis in fungi. Fungal Genet Biol 40: 187-196.

Walton, F.J., Heitman, J., and Idnurm, A. (2006) Conserved elements of the RAM signaling pathway establish cell polarity in the basidiomycete Cryptococcus neoformans in a divergent fashion from other fungi. Mol Biol Cell 17: 3768-3780.

Wei, H., Requena, N., and Fischer, R. (2003) The MAPKK kinase SteC regulates conidiophore morphology and is essential for heterokaryon formation and sexual development in the homothallic fungus Aspergillus nidulans. Mol Microbiol 47: 1577-1588.

Wei, X., Shimizu, T., and Lai, Z.C. (2007) Mob as tumor suppressor is activated by Hippo kinase for growth inhibition in Drosophila. Embo J 26: 1772-1781.

Weiss, E.L., Kurischko, C., Zhang, C., Shokat, K., Drubin, D.G., and Luca, F.C. (2002) The Saccharomyces cerevisiae Mob2p-Cbk1p kinase complex promotes polarized growth and acts with the mitotic exit network to facilitate daughter cell-specific localization of Ace2p transcription factor. J Cell Biol 158: 885-900.

Wolfe, B.A., and Gould, K.L. (2005) Split decisions: coordinating cytokinesis in yeast. Trends Cell Biol 15: 10-18. 
Xu, T., Wang, W., Zhang, S., Stewart, R.A., and Yu, W. (1995) Identifying tumor suppressors in genetic mosaics: the Drosophila lats gene encodes a putative protein kinase. Development 121: 1053-1063.

Yarden, O., Plamann, M., Ebbole, D.J., and Yanofsky, C. (1992) cot-1, a gene required for hyphal elongation in Neurospora crassa, encodes a protein kinase. Embo J 11: 2159-2166.

Zallen, J.A., Peckol, E.L., Tobin, D.M., and Bargmann, C.I. (2000) Neuronal cell shape and neurite initiation are regulated by the $\mathrm{Ndr}$ kinase $\mathrm{SAX}-1$, a member of the Orb6/COT-1/warts serine/threonine kinase family. Mol Biol Cell 11: 3177-3190. 


\title{
CHAPTER IV
}

\section{Activation of the NDR kinase COT1 involves the GC kinase POD6, MOB co-activators and distinct phosphorylation events}

\begin{abstract}
NDR kinases are involved in growth and differentiation. For their function they require the association with $\mathrm{MOB}$ proteins and phosphorylation of two conserved residues in the activation segment and the hydrophobic motif, yet the individual steps of activation and their hierarchical order are not fully resolved. Here we show that the NDR kinase COT1 is autophosphorylated in cis at Ser417 of the activation segment, while the germinal centre kinase POD6 is involved in the phosphorylation of Thr589 in the hydrophobic motif. The interaction of MOB2 proteins with COT1 is independent of COT1 activity, its phosphorylation state of the two regulatory residues, and does not require POD6. In vitro autophosphorylation and COT1 activity correlate, but both assays do not mirror the in vivo functionality of COT1 as determined by the growth rate of mutant strains. This discrepancy is explained by a multi-step activation model that includes a conformational change induced by the two phosphorylation events and altered localization of the COT1MOB2 complex.
\end{abstract}




\section{Introduction}

Nuclear Dbf2p-related (NDR) kinases represent a subfamily of AGC (protein kinase $\underline{A}$, PKG and PKC-like) kinases. These kinases share structural similarities and require phosphorylation of a conserved Ser/ Thr residue within the activation segment of the kinase domain for their activity (Johnson et al., 1996). Activation of many AGC kinases also requires a second phosphorylation event or the permanent presence of an acidic residue in a hydrophobic motif that is located $45-60$ residues C-terminal of the catalytic kinase core in addition to phosphorylation of the activation loop (Keshwani \& Harris, 2008, Keranen et al., 1995, Biondi \& Nebreda, 2003). The phosphorylated hydrophobic motif interacts with a hydrophobic pocket in the $\mathrm{N}$-terminal lobe of the kinase domain and induces a reconfiguration of the bilobal kinase structure and increased catalytic activity (Frodin et al., 2002, Yang et al., 2002a, Biondi et al., 2000, Engel et al., 2006). Depending on the presence of the two motifs within one AGC kinase or not, this interaction can occur in an intra- or intermolecular manner. Furthermore, the chronology of these two phosphorylation events depends on the specific kinase, and both phosphorylation of the activation loop or hydrophobic motive phosphorylation - can occur as the first step of kinase activation (Shah \& Hunter, 2004, Gao et al., 2001)

Work in fungal and animal cells has resulted in emerging signalling networks (Kanai et al., 2005, Nelson et al., 2003, Saucedo \& Edgar, 2007, Hergovich et al., 2006b) that includes an NDR kinase and its binding partner and co-activator $\mathrm{MOB}$, which is involved in membrane localization of the complex and activation of the kinase by promoting autophosphorylation of its activation segment (Hergovich et al., 2005, Lai et al., 2005, Wei et al., 2007). NDR activity is also controlled through upstream Ste20-type kinases of the germinal centre (GC) kinase family (Dan et al., 2001, Pombo et al., 2007), which phosphorylate NDR at the hydrophobic motif (Emoto et al., 2006, Hirabayashi et al., 2008, Stegert et al., 2005, Vichalkovski et al., 2008). Whether this sequential and strictly hierarchical organization of the two kinases, and of the two phosphorylation events represent the complete picture is still unclear, as genetic data from several systems suggest feedback loops and parallel signalling activities of both kinases (Emoto et al., 2006, Gallegos \& Bargmann, 2004, Seiler et al., 2006, Stegert et al., 2005). In particular, the detailed function of MOB in the initial activation step of NDR is not clearly defined. 
Several reports have indicated that phosphorylation of MOB1 by the upstream acting GC kinase is important for promoting the MOB1-NDR interaction (Hirabayashi et al., 2008, Praskova et al., 2008, Wei et al., 2007), yet this has not been shown for the interaction of an NDR kinase with MOB2-type adaptors. Thus the general nature of this activation mechanism is currently unclear. This issue is further complicated by the fact that a single NDR kinase is able to associate with MOB1- and MOB2-type adaptors in animal cells (Bichsel et al., 2004, He et al., 2005a, Devroe et al., 2004). Furthermore, recent work on fungal NDR kinases has shown that phosphorylation of the activation loop is critical for kinase activity, but is only partially required for in vivo function, while phosphorylation of the C-terminal hydrophobic motif is required in vivo, but not for kinase activity (Hou et al., 2004, Jansen et al., 2006, Ziv et al., 2009), illustrating the problem that the prevailing view of NDR activation, which is based largely on in vitro structural analyses and enzyme activities, and the in vivo function of these kinases do not correlate. Thus, we do not yet fully understand the function of these critical phospho-sites, the timely order of activation loop and hydrophobic motif phosphorylation and their biological consequences in the activation mechanism of NDR kinases.

The NDR kinase COT1 of the ascomycete Neurospora crassa is the founding member of this kinase family and is regulated by the GC kinase POD6 and two interacting MOB2-type proteins (Seiler et al., 2006, Yarden et al., 1992, Maerz et al., 2009) COT1, POD6 and $\mathrm{MOB} 2 \mathrm{~A} / 2 \mathrm{~B}$ are required for polar cell elongation, but not for the establishment of polarity per se. Strains with mutations in these central COT1 complex components are viable and display hyperbranched cell growth, indicating that the COT1 pathway is essential for hyphal tip extension, and is required to restrict excessive branch formation in subapical regions of the cell. A similar branching and growth-termination phenotype has been observed in neuronal cells of NDR kinase mutants (Emoto et al., 2004, Emoto et al., 2006, Geng et al., 2000, Zallen et al., 2000, Gallegos \& Bargmann, 2004), suggesting an evolutionarily conserved function of NDR kinases in the formation of branched cellular structures. Ser417 and Thr589 in the activation loop and hydrophobic motif of COT1, respectively, are two key regulatory phosphorylation sites that regulate polar growth and hyphal branch initiation by altering cell wall integrity and actin organization (Ziv et al., 2009). Furthermore, the activity of COT1 is controlled through two MOB2-type proteins. The interaction with COT1 requires a conserved region directly preceding the kinase 
domain of COT1, which is sufficient for the formation of COT1-MOB2 heterodimers, but also for kinase homodimerization (Maerz et al., 2009). An additional N-terminal extension that is poorly conserved, but present in most fungal NDR kinases, is required for further stabilization of both types of interactions and for stimulating COT1 activity. COT1 lacking this region is degraded in a mob-2 background. Here we dissect the mechanism of COT1 auto- and hydrophobic motif phosphorylation and provide a multi-step model for the activation of COT1 that may also help to explain the available data on the regulation of NDR kinases in other organisms. 


\section{Results and Discussion}

\section{Ser417 is the major autophosphorylation site of COT1}

When immunoprecipitated (IPed) COT1 that was myc-tagged at its endogenous locus under the control of its endogenous promoter was subjected to in vitro phosphorylation reactions, we detected ${ }^{32} \mathrm{P}$ incorporation in both COT1 isoforms (Figure IV-1 A). The substitution of an aspartic acid that is essential for catalytic activity of AGC and NDR kinases (Hanks \& Hunter, 1995, He et al., 2005b, Jansen et al., 2006) abolished phosphate incorporation in precipitated myc-COT1(D337A), indicating that this was indeed the result of COT1 autophosphorylation and not phosphorylation of COT1 by a co-purifying kinase. AGC kinases can autophosphorylate at Ser/ Thr residues of the activation segment or the hydrophobic motif during activation (Behn-Krappa \& Newton, 1999, Stegert et al., 2004). When we used peptides corresponding to COT1(409-425) and COT1(576-598) covering the activation segment and hydrophobic motif of COT1 as in vitro substrates, we detected phosphate incorporation in COT1(409-425), but not COT1(576-598) (Figure IV-1 B). Thus COT1 autophosphorylation occurred within the activation segment and not the hydrophobic motif. To differentiate between Ser417 and the neighbouring Thr418 as site of autophosphorylation, we used strains that carried substitutions of Ser417 to almandine and Ser417 to glutamate of the endogenous cot-1 gene; as control we used strains mutated at Thr589 within the hydrophobic motif in a corresponding manner (Ziv et al., 2009). Autophosphorylation experiments with precipitants of these COT1 variants identified Ser417 as the only autophosphorylation site of COT1 (Figure IV-1 C). We also detected autophosphorylation of COT1 in vivo by probing the precipitated kinase with a phospho-Ser417 specific NDR antibody (Figure IV-1 C). Interestingly, we observed equal labelling of both COT1 isoforms by the phospho-Ser417 antibody. This contrasted with the in vitro ${ }^{32} \mathrm{P}$ incorporation that occurred primarily in the large isoform and suggested additional levels of regulation, e.g. by phosphatases, occurring in vivo. We conclude that Ser417 within the activation loop is the major/only site of COT1 autophosphorylation. These experiments also indicated that Ser417 phosphorylation is independent of Thr589 modification within the hydrophobic motif. 
Activation segment exchange is a common mechanism for autophosphorylation of kinase dimers in trans (Oliver et al., 2007, Pike et al., 2008). COT1 and other NDR kinases are able to dimerize (Hou et al., 2004, Nelson et al., 2003, Maerz et al., 2009), and we explored the possibility of autophosphorylation occurring in cis or trans of a COT1 dimer by generating heterokaryons of myc-cot-1(D337A) with a his-3 strain harbouring an untagged wild type copy of cot-1 (Figure IV-1 D). No Ser417 phosphorylation was detected in COT1 precipitated from myc-cot-1(D337A) and from the myc-cot-1(D337A) + his- $3^{-}$ heterokaryotic strain. These data strongly suggest that Ser417 phosphorylation in vivo is performed in cis. The role of dimer formation for the function of the kinase remains unclear, but it might be possible that dimerization inhibits autophosphorylation and the kinase is kept in an inactive state by dimerization.

A IP: $\alpha$-myc autoradiograph

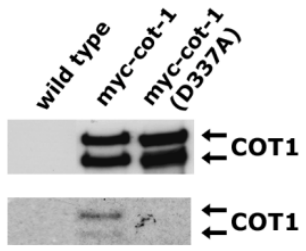

B

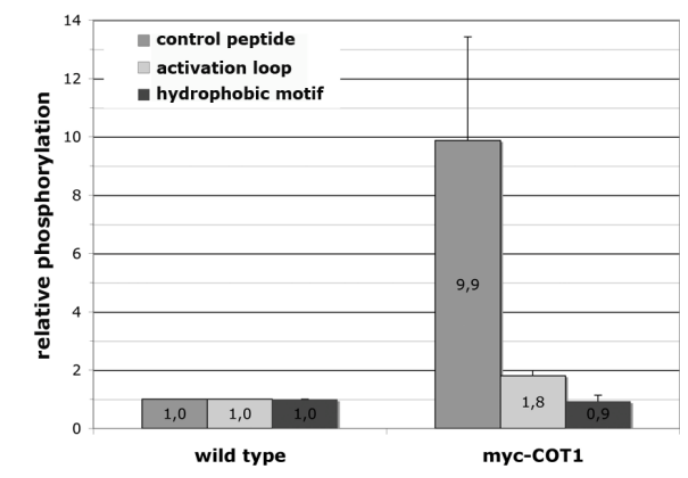

D

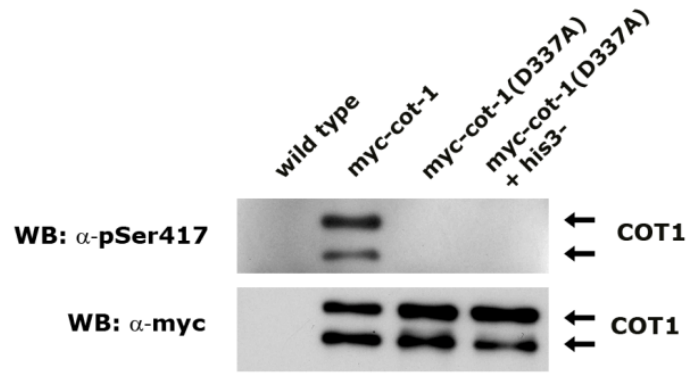
autoradiograph

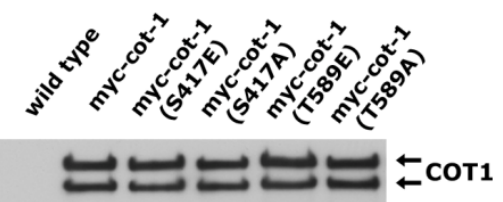

WB: $\alpha-\mathbf{m y c}$

WB: $\alpha-$ per417

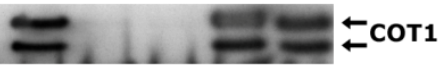

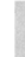

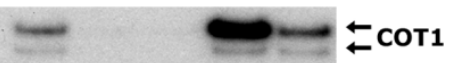

Figure IV-1 Ser417 is the major site of COT1 autophosphorylation. (A) Immunoprecipitated myc-COT1 variants from the indicated strains were probed with anti-myc antibody (upper panel) to determine equal amounts of precipitated kinase and subjected to an ${ }^{32} \mathrm{P}$ in vitro autophosphorylation reaction (lower panel). (B) In vitro kinase assays with the indicated peptides as artificial substrates ( $n=3$ ). (C) Immunoprecipitated myc-COT1 variants from the indicated strains were probed with anti-myc antibody (upper panel), P-Ser417 specific antibody (middle panel), and subjected to an in vitro autophosphorylation reaction (lower panel). (D) myc-COT1 was precipitated from the indicated strains, tested probed via P-Ser417 specific antibody (upper panel) and anti-myc antibody (lower panel). 


\section{POD6 is involved in hydrophobic motif phosphorylation of COT1}

We have recently shown that myc-COT1(T589E) did partially suppress the growth defects of a conditional pod-6(ts) strain (Ziv et al., 2009). To obtain quantitative data in a deletion background, we crossed the myc-cot-1 phospho-site alleles into $\Delta$ pod-6. The growth rate of $\Delta$ pod-6;myc-cot-1(T589E) was $29 \%$ of myc-cot-1 $(n \geq 5)$, while tip extension was not measurable in $\Delta$ pod-6;myc-cot-1(T589A), $\quad \Delta$ pod-6;myc-cot-1(S417E), $\Delta$ pod-6;myc-cot1(S417A), which all displayed phenotypic characteristics identical to $\Delta p o d-6$ (Figure IV-2). These genetic data clearly indicate a role for POD6 in the hydrophobic motif phosphorylation of COT1. Additional functions of POD6 in the regulation of the COT1 pathway are suggested by the fact that the in vivo functionality of $\Delta$ pod-6;myc-cot1(T589E) was only $46 \%$ of myc-cot-1(T589E).

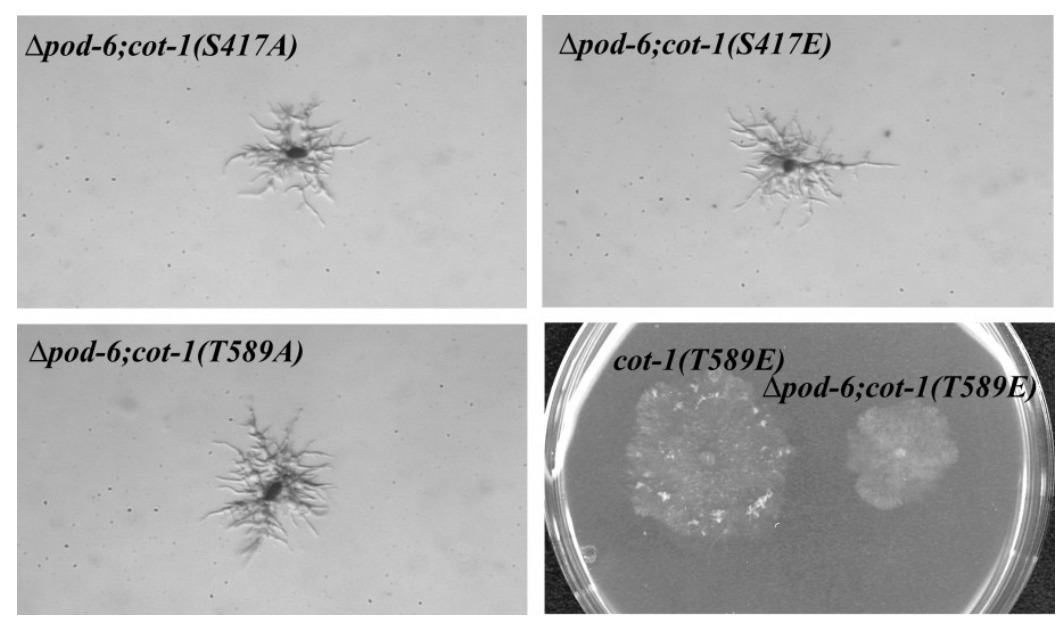

Figure IV-2 POD6 is involved in Thr589 phosphorylation of COT1. Suppression analysis of $\Delta p o d-6$ in the indicated cot-1 strains

\section{The interaction of COT1 with MOB2 does not require functional COT1 or the presence of POD6}

The association of COT1 with MOB2A/2B is essential for COT1 function in vivo and for kinase activity in vitro (Maerz et al., 2009). The phospho-status of COT1 may regulate its association with $\mathrm{MOB}$ proteins and may thus explain the altered in vitro activities observed in the different myc-COT1 variants (Ziv et al., 2009). We tested the COT1 phospho-variants in co-IP experiments for their capability to interact with MOB2A and MOB2B, but did not observe any change in COT1-MOB association (Figure IV-3 A). When 
we precipitated COT1 from the kinase dead myc-COT1(D337A) strain that lacked ${ }^{32} \mathrm{P}$ and P-Ser-antibody-based autophosphorylation, we were able to co-IP wild type levels of associated MOB2A and MOB2B (Figure IV-3 B). Thus, COT1 activity and autophosphorylation is no prerequisite for MOB interaction, and MOB binding and Ser417 phosphorylation are independent events that precede kinase activation. In line with this conclusion was the fact that the СОT1-MOB interaction was also unaffected in a cot-1(ts) strain carrying a His351 to arginine substitution, which conferred a temperature-sensitive growth defect (Gorovits et al., 1999) and lacked detectable in vitro kinase and autophosphorylation activity at permissive and restrictive conditions (suppl. Figure S IV1).

A

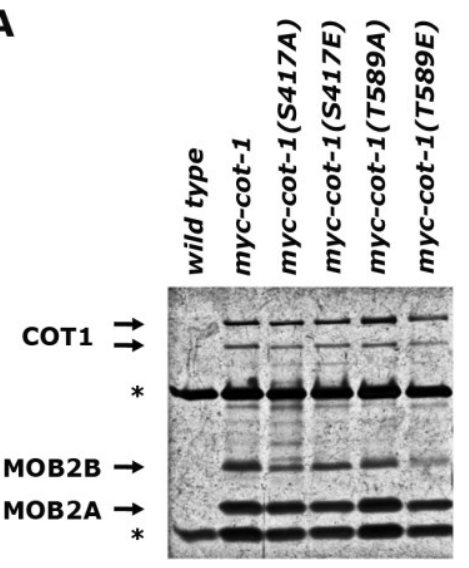

B

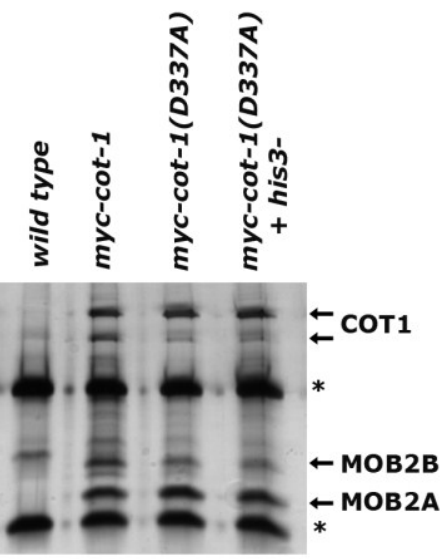

C
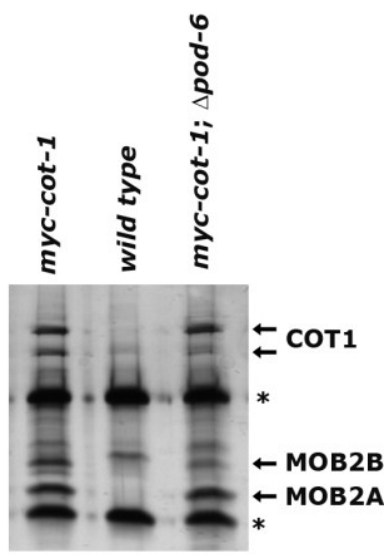

Figure IV-3 COT1-MOB2 interaction does not require functional COT1 or the upstream kinase POD6. Co-IP experiments of СОТ1-associated МОВ2 proteins in the indicated phospho-site mutants $(A)$, the kinase inactive strain myc-cot-1(D337A) (B), and a $4 p o d-6$ background (C). myc-COT1 was immunoprecipitated from the indicated strains using anti-myc antibody and subsequently used for silver staining; ${ }^{*}$ indicates the heavy and light chain of the antibody.

Phosphorylation of MOB1 through upstream GC kinases has been shown to increase the affinity of MOB for NDR (so far only shown for members of the MOB1 subfamily, not for MOB2 proteins; (Hirabayashi et al., 2008, Praskova et al., 2008, Wei et al., 2007). We tested if the interaction of myc-СОT1 with the two MOB2 proteins was abolished in $\Delta p o d$ 6, but still detected wild type levels of associated MOB2 proteins (Figure IV-3 C). This was consistent with close to wild type levels of myc-COT1 activity precipitated from a $\Delta p o d-6$ background (Table IV-1) and comparable rates of COT1 autophosphorylation detected by the phospho-Ser417 antibody (Figure IV-4 A). Interesting was that we also detected wild type levels of in vivo Ser417 autophosphorylation in a $\Delta m o b-2 a ; \Delta m o b-2 b$ double deletion 
strain despite almost absent COT1 in vitro activity (Figure IV-4 B). The small COT1 isoform is not stable in a $\Delta m o b-2 a ; \Delta m o b-2 b$ double deletion background and thus cannot be detected (Maerz et al., 2009).

A

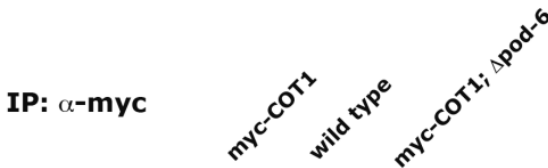

WB: $\alpha-p S e r$

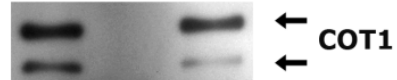

WB: $\alpha-$ myc

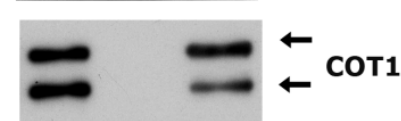

B

IP: $\alpha-\mathbf{m y c}$

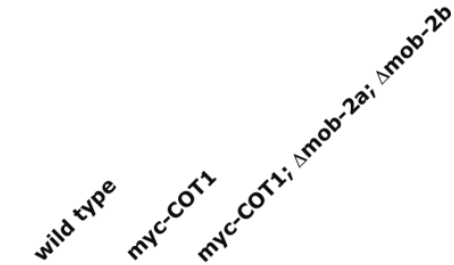

WB: $\alpha-p S e r$

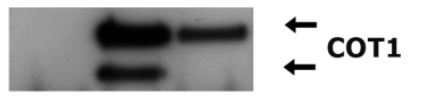

WB: $\alpha-$ myc

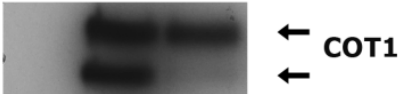

Figure IV-4 auto-P und P-Ser417 Western in pod-6 und mob deletion strains. myc-COT1 was immunoprecipitated from the indicated strains using. Precipitates were probed with P-Ser417 specific antibody and anti-myc-antibody to determine the auto-P in $\Delta p o d-6$ (A) and $\Delta m o b-2 a ; \Delta m o b-2 b$ (B) background strains.

Thus, COT1-MOB interaction and COT1 kinase activity is not regulated through POD6 and independent of hydrophobic motif phosphorylation. Nevertheless, all these strains are non-functional for COT1 signalling, indicated by their identical hyperbranching phenotypes. Taken together, these results indicate that the association of COT1 with MOB2A/B is not regulated. The interaction is independent of the COT1 phosphorylation state, its kinase activity and does not require the presence of POD6. This contrasts with the phosphorylation of MOB1-type proteins by the upstream GC kinases MST1/2 or Hippo in animals that were shown to drive the association of MOB1 with NDR kinases (Hirabayashi et al., 2008, Praskova et al., 2008, Wei et al., 2007). Thus, this mode of regulation may be exclusive for MOB1. However, this does not exclude the possibility that phosphorylation of MOB2-type adaptors through GC kinases may regulate the subcellular localization of the MOB2-NDR complex. Human MOB proteins were shown to target NDR kinases to the plasma membrane (Hergovich et al., 2005, Hergovich et al., 2006a), raising the hypothesis that membrane targeting of the NDR-MOB complex is regulated by MOB phosphorylation through the upstream GC kinase. 


\section{In vitro COT1 activity does not correlate with its in vivo function}

The in vitro kinase activities of the mutant myc-COT1 proteins obtained using a synthetic peptide substrate commonly used for NDR kinases correlated with their ${ }^{32} \mathrm{P}$-based autophosphorylation characteristics (Table IV-1). However, the in vivo phospho-Ser417 status was different, indicating that in vitro obtained kinase activities alone do not allow establishing a coherent activation schema for NDR. This is consistent with similar observations in the few available comparative analyses of related NDR kinases in both yeasts and Drosophila (He et al., 2005b, Hou et al., 2004, Jansen et al., 2006), but mechanistic explanations for these discrepancies are currently lacking. We used the fungal growth rate as a quantitative measure for in vivo functionality of modified COT1 (Table IV-1). Altering Ser417 to mimic either phosphorylated or non-phosphorylated protein lead to a reduction, while modification of Thr589 in either direction increased the in vitro activity of myc-COT1. myc-COT1(S417A) had almost no in vitro activity in the peptide assay, but the strain displayed a reasonable growth rate (32\% of myc-COT1), while myc-COT1(T589A) had 2.5-fold increased in vitro activity, but grew poorly (13\% of myc-COT1). Furthermore, the activity of myc-COT1(S417E) was reduced to $19 \%$, while myc-COT1(T589E) had ca 23-fold increased activity, but both strains grew reasonably well ( $81 \%$ and $63 \%$ of myc-COT1, respectively). Thus, phosphorylation of the hydrophobic motif is more important for the in vivo function of COT1 than its autophosphorylation. This is consistent with the data obtained in $\Delta$ pod-6, which displayed $80 \%$ COT1 activity and normal rates of autophosphorylation, but no growth. Furthermore, abolishing COT1's ability to autophosphorylate in myc-COT1(S417A) und deletion of the two mob-2 genes resulted in kinase activities barely above background, but the functionality of the two strains was quite different. The growth rate of myc-cot-1(Ser417A) was reduced to $32 \%$, while loss of $\Delta m o b-2 a ; \Delta m o b-2 b$ abolished functional signalling and the strain displayed $\Delta \cot -1$ defects (Table IV-1). Thus, the growth tests indicate that the in vivo functionality of СОT1 is more compromised by deleting the two mob-2 genes than interfering with the autophosphorylation of COT1. An attractive hypothesis for the apparent discrepancies between in vitro kinase activity and in vivo functionality of COT1 may be that the artificial 
substrate used for the in vitro kinase assays does reflect the phosphorylation competence of the kinase, but not the actual phosphorylation of an endogenous substrate. We hypothesize that phosphorylation of Ser417 within the activation segment makes COT1 catalytically competent and partially active towards an artificial substrate. This allows the subsequent phosphorylation of Thr589 through an upstream kinase and enables the kinase to phosphorylate its in vivo substrates. This step-wise activation would explain why myc-COT1 purified from myc-cot-1(T589A) and myc-cot-1; $\Delta$ pod-6 has reasonable in vitro kinase activities, but grow poorly. Also the highly reduced kinase activity of COT1(S417A), but the retained in vivo function of myc-cot-1(S417A) is in agreement with the presented hypothesis. 
Table IV-1 Summary of COT1 autophosphorylation, kinase activity and relative growth

\begin{tabular}{|c|c|c|c|c|c|}
\hline & $\begin{array}{c}\text { In vitro auto- } \\
\text { phosphorylation of COT1 } \\
(n \geq 3 \pm S D)\end{array}$ & $\begin{array}{c}\text { In vivo Ser417 } \\
\text { phosphorylation of } \\
\text { СОT1 }\end{array}$ & $\begin{array}{l}\text { COT1 - MOB } \\
\text { interaction }\end{array}$ & $\begin{array}{l}\text { Peptide-based COT1 } \\
\text { activity }(n \geq 3 \pm S D)\end{array}$ & $\begin{array}{l}\text { Relative growth rate at } 25^{\circ} \mathrm{C} \\
\qquad(\mathrm{n} \geq 5 \pm S D)\end{array}$ \\
\hline myc-cot-1 & + & $+^{1}$ & + & $100 \%$ & $100 \%$ \\
\hline myc-cot-1(D337A) & - & - & + & $0 \%$ & $0 \%^{2}$ \\
\hline$m y c-c o t-1(S 417 A)$ & - & - & + & $1 \pm 1 \%^{3}$ & $32 \pm 2 \%$ \\
\hline myc-cot-1(S417E) & - & - & + & $19 \pm 7 \%^{3}$ & $81 \pm 4 \%$ \\
\hline myc-cot-1(T589A) & + & $++^{1}$ & + & $251 \pm 158 \%^{3}$ & $13 \pm 3 \%$ \\
\hline myc-cot-1(T589E) & $\begin{array}{c}2 \pm 1 \text { fold increased }^{4} \\
+ \\
4 \pm 1 \text { fold increased }\end{array}$ & $+^{1}$ & + & $2318 \pm 1040 \%^{3}$ & $63 \pm 3 \%$ \\
\hline $\begin{array}{l}m y c-c o t-1 ; \Delta m o b- \\
2 a ; \Delta m o b-2 b\end{array}$ & - & $+^{5}$ & - & $1 \pm 0.5 \%^{6}$ & $0 \%^{2}$ \\
\hline$\Delta p o d-6 ; m y c-c o t-1$ & not determined & $+^{7}$ & + & $80 \pm 23 \%$ & $0 \%^{2}$ \\
\hline $\begin{array}{l}\Delta p o d-6 ; m y c-c o t-1 \\
(T 589 E)\end{array}$ & not determined & + & + & not determined & $29 \pm 5 \%$ \\
\hline
\end{tabular}

${ }^{1}$ equal phosphorylation of large and small COT1 isoforms in vivo

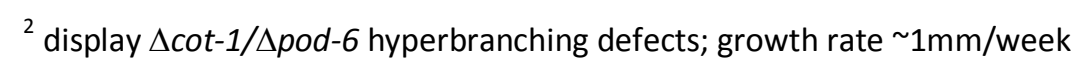

${ }^{3}$ data from Ziv et al., 2009

${ }^{4}$ in vitro $\mathrm{P}^{32}$ incorporation in both isoforms, but only phosphorylation of large COT1 isoform increased

${ }^{5}$ only large isoform of COT1 expressed and phosphorylated

${ }^{6}$ data from Maerz et al., 2009

${ }^{7}$ phosphorylation of lower isoform reduced, but difficult to quantify, due to the high background generated by the anti-phospho-Serine antibody 


\section{A conformational change induced through hydrophobic motif phosphorylation is required for full activation of COT1}

Sequence analysis of COT1 and selected AGC kinases revealed the conservation of all residues involved in the interaction of the phosphorylated hydrophobic motif with the hydrophobic pocket in COT1 (Figure IV-5 A). Thus, we tested if a conformational change induced by hydrophobic motif phosphorylation could activate COT1 and generated peptides covering region 576-598 containing substitutions of Thr589 to aspartate or alanine to mimic phosphorylated or non-phosphorylated hydrophobic motif (termed HMD and HM-A, respectively). We observed 2-fold increased myc-COT1 activity in the presence of $1 \mathrm{mM} H M-D$, while the addition of $1 \mathrm{mM}$ HM-A to the kinase assays reduced its activity by $21 \%$ (Figure IV-5 B). As predicted, myc-COT1(T589E) was not stimulated by adding HM-D, but the addition of HM-A reduced the activity to $82 \%$ of the control. The opposite pattern was observed in kinase assays with myc-COT1(T589A): addition of HM-D increased kinase activity 2.5-fold, while the addition of HM-A did not alter it. Thus, a peptide mimicking the phosphorylated hydrophobic motif is able to induce a conformational change in the COT1 structure in trans that leads to increased kinase activity, while HM-A showed an inhibitory effect. In line with these biochemical data, we also observed that myc-cot-1(T589A), but none of the other phospho-site mutants displayed a temperature-sensitive growth defect (Figure IV-5 C), providing further indication of a conformational change of COT1, which is induced by phosphorylation of Thr589.

Because myc-COT1(S417A) and myc-COT1(S417E) displayed low in vitro kinase activities in the control experiments (Table 1), we were unable to determine the effects of the HM-A and HM-D peptides unequivocally. The low kinase activities of myc-COT1(S417E) and mycCOT1(S417A) lead to high variability during measurement of ${ }^{32} \mathrm{P}$ incorporation. The resulting high standard derivation makes it nearly impossible to provide a significant conclusion onto the behaviour of these two proteins. However, despite the high variability between the different assays, we observed the tendency that the kinase activities of myc-COT1(S417E) by adding HM-D and HM-A follows the same pattern as shown for myc-COT1 (Figure IV-5 B), The activity of myc-COT1(S417A) was barely above background in the control experiments and did not change significantly when either peptide was added. These data indicate that the reorganization of the kinase structure 
induced through HM-phosphorylation may require the autophosphorylation of Ser417.

This is consistent with data obtained in other AGC kinases such as PKC, in which activation loop phosphorylation is required to transfer the kinase in a so-called catalytically competent state (Toker \& Newton, 2000, Newton, 2003). This first phosphorylation event is a prerequisite for further phosphorylation that leads to a fully active kinase.

A

COT1 (short)
HsRSK2

HsNDR1

$\mathrm{MmPKA \alpha}$

\begin{tabular}{l}
\hline hydrophobic pocket \\
\hline
\end{tabular}

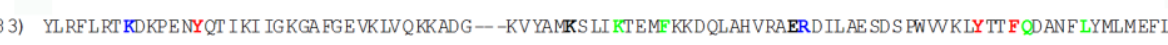

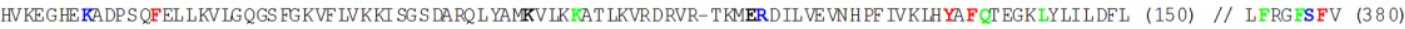
FL RLKRT RLGLE DFES LKV IGRGA FGEVRLVQKKDTG --HVYAMK I LRKADMLEKE QVGH I RAERDILVEADS LWWKMFYS F ODKLNLYLIMEFL (171) // VF TNYTYK (446)

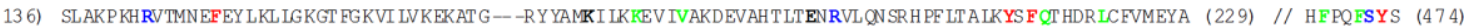
(31) WE TP S QNTAQLD QFD DI KT LGTGS F GR WLVKHKE SG --NH YAMKL LDKQKVVKLKQIE HTLNEKR ILQAVNF PFLVKLEF SFKDNSNLYMVMEYV (124) // EFTE F* (351)
B

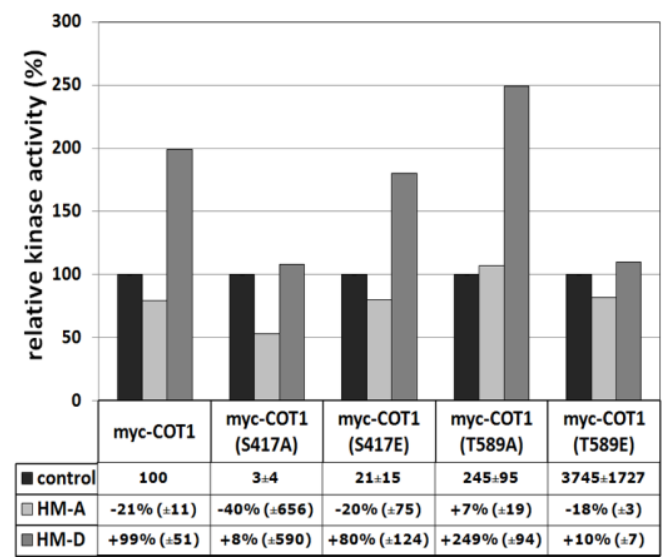

C

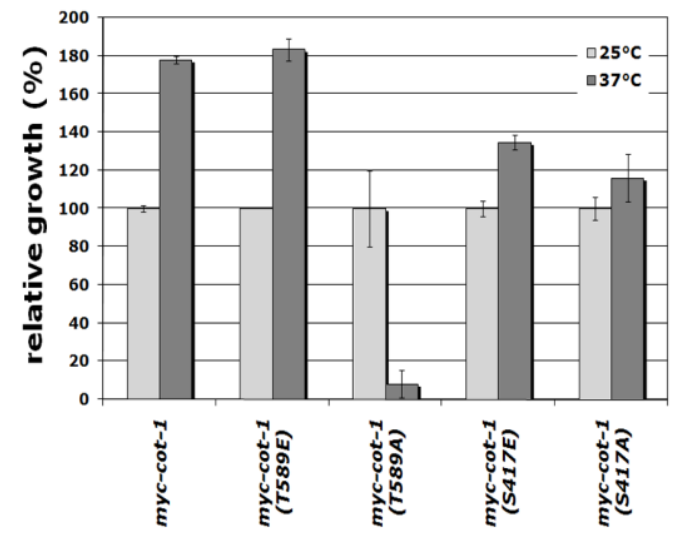

Figure IV-5 A conformational change induced through hydrophobic motif phosphorylation is required for activation of COT1. (A) Alignment of COT1 and selected AGC kinases, emphasizing residues that are important for the interaction between the hydrophobic pocket within the small lobe of the kinase domain and the C-terminal phosphorylated hydrophobic motif (Frodin et al., 2002, Yang et al., 2002b). green: residues that bind the first two Phe/Tyr of the hydrophobic motif; blue: residues that bind the phosphate of the hydrophobic motif; red: residues that bind the last Phe/Tyr of the hydrophobic motif; black: ion pair. (B) in vitro kinase activities of myc-COT1 variants precipitated from the indicated strains. Relative activity of the control was set to $100 \%$, the activity of reaction mixtures containing HM-A or HM-D were calculated relative to the respective control. One typical experiment is shown. Below in the control row the relative activity of the indicated myc-COT1 variant from $n \geq 3$ experiments with the standard derivation relative to myc-COT1 are indicated. For HM-A and HM-D the percental increase 
or reduction of the kinase activity relative to the respective control with standard derivation are shown $(n \geq 3)$. (C) Relative growth rates of the indicated strains at $25^{\circ} \mathrm{C}$ and $37^{\circ} \mathrm{C}$.

\section{Summarizing model: COT1 activation involves multiple steps}

Based on the results presented here for COT1 and available data on other NDR kinases, we propose the following model for COT1 activation that summarizes our results and is consistent with available data on the regulation of NDR kinases in other organisms (Table IV-1; summarizing Figure IV-6). We believe that inactive COT1 is forming a dimer this has been shown for several other fungal NDR kinases (Hou et al., 2004, Nelson et al., 2003), and we propose that the transition between the COT1-СОT1 homo- and the COT-MOB heterodimer is the first step in the activation of the kinase. The COT-MOB heterodimerization together with autophosphorylation of COT1 at Ser417 in the activation segment in cis may relieve the autoinhibition of the COT1 homodimer and may lead to basal activity and. It may also contribute to the correct localization of COT1. This transition seems to be regulated via competing interaction sites, and may involve (but is at least in the case of COT1 not fully dependent on) phosphorylation of MOB by the GCK POD6. This model also predicts that the loss of COT1 function in $\Delta p o d-6$ is the result of non-phosphorylated COT1-Thr589, which is probably unable to bind to and to phosphorylate endogenous substrate(s). In addition, POD6 may also be involved in the correct localization of the COT1-MOB2 complex. The subsequent phosphorylation of Thr589 through POD6 results in full activation of COT1. This final phosphorylation event is 
likely to include the restructuring of the protein induced by the interaction of the phosphorylated hydrophobic motif with the hydrophobic pocket as described for other AGC kinases (Frodin et al., 2002; Yang et al., 2002). We propose that this conformational change leads to the ability to interact or at least to a more efficient interaction with and phosphorylation of endogenous COT1 substrates. The model does not explain the decreased in vitro activity observed of COT1(S417E). Thus makes it likely that further regulatory steps are necessary to achieve a fully active kinase such as COT1(T589E). This might hypothetical include a phosphorylation/dephosphorylation cycle of Ser417, but this is highly speculative. However, this is not a limiting step and might be unimportant for the in vivo function of COT1.

This prediction of coupling the synergistic regulation of kinase activity by two-step phosphorylation and a conformational change resulting in altered kinase-substrate interaction would explain the observed discrepancy of in vitro kinase activity and in vivo defect of the phospho-site NDR kinase variants in our and other systems (He et al., 2005b, Hou et al., 2004, Jansen et al., 2006, Ziv et al., 2009). This model, does not answer the increased kinase activity of COT1(T589A), and only partially explains the reduced kinase activity of COT1(S417E), but is providing a basis for further dissection of the individual steps of NDR kinase activation in future structural analyses. 


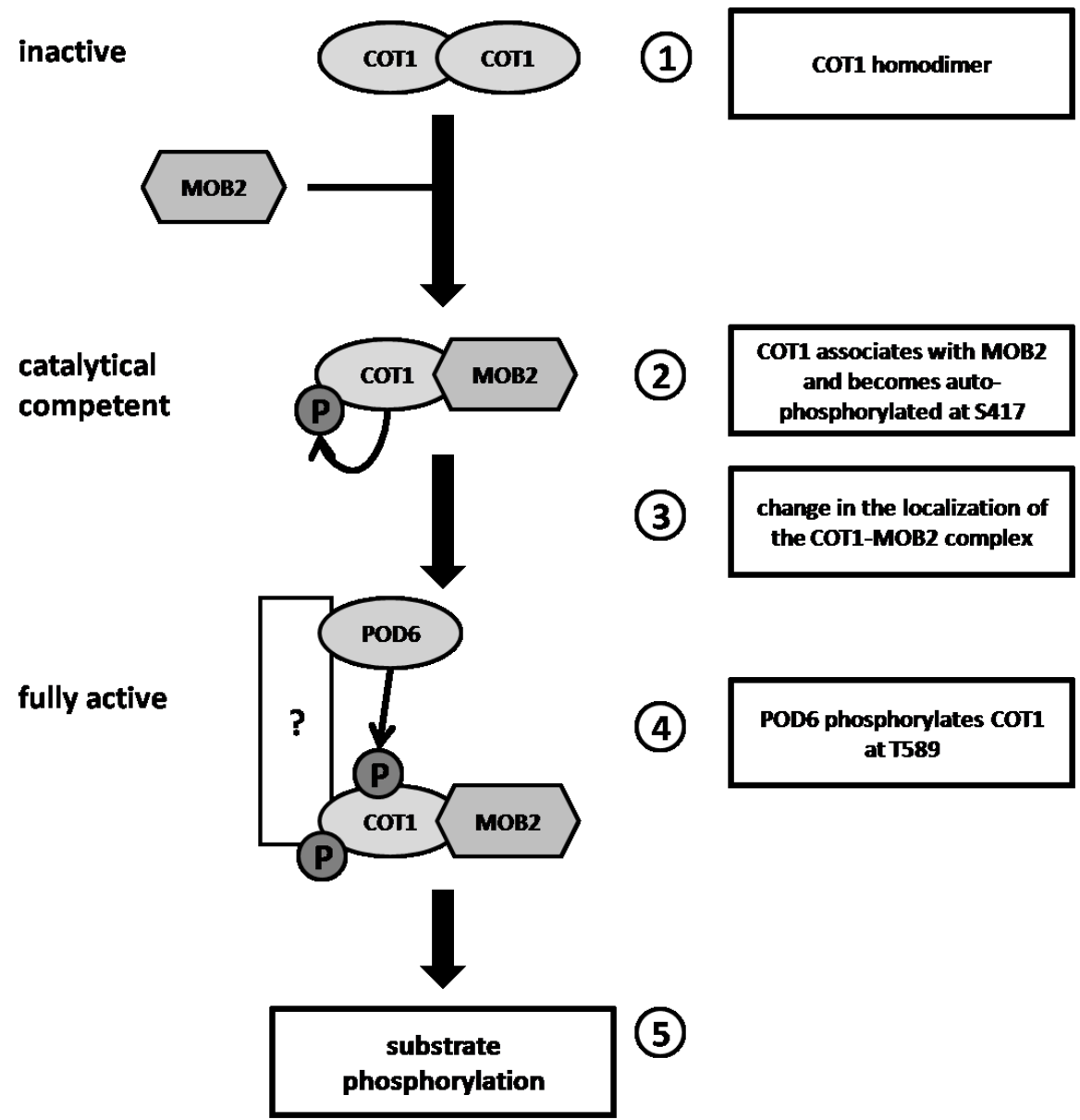

Figure IV-6 Model for the stepwise activation of COT1. See text for details. 


\section{Material and Methods}

\section{Strains, constructs and growth conditions}

Strains used in this study are listed in Table IV-2 (see also McCluskey, 2003). General genetic procedures and media used in the handling of $N$. crassa have been described (Davis \& DeSerres, 1970, Davis, 2000) or are available through the Fungal Genetic Stock Center (www.fgsc.net). Growth rates of fungal strains were determined by measuring radius of colonies on agar plates starting with a well-established colony to exclude the lag phase of germination and the initial slow growth phase of a developing colony. Microscopic documentation of fungal hyphae or colonies was performed with an SZX16 stereomicroscope, equipped with a Colorview III camera and Cell ${ }^{D}$ imaging software (Olympus, Japan) or an ORCA ER digital camera (Hamamatsu, Japan) mounted on an Axiovert S100 microscope (Zeiss, Germany). Image acquisition was done using the Openlab 5.01 software (Improvision, Great Britain) and images were further processed using Photoshop CS2 (Adobe, USA).

To generate the cot-1(ts)-myc fusion construct, plasmid pME8 [previously described in (Seiler et al., 2006)]. was used as a template for PCR amplification using the primers 5GCA TCG ATT TAA ACA TAT GGA GCA-3 and 5-GTG ATT ATA CAT ATG AGG CCT tta A-3, which introduced a new stop codon (indicated by lower case letters) and an Ndel restriction site (underlined) at the PCR product ends, thereby facilitating the cloning of the amplicon into the Ndel site in pCZ13 (which is the genomic Smal/EcoRI cot-1 fragment from pOY18 ligated into pUC118) and creating pCZ22 (cot-1(wt)-myc). Correct integration of the MYC tag sequence at the $3{ }^{`}$ end of the cot-1 gene-coding region was verified by sequencing. A COT1(ts)::MYC fusion was constructed by replacing the wild-type catalytic domain (850 bp) of pCZ22 with the corresponding domain of the cot-1(ts) mutant allele, that was amplified from cot-1 genomic DNA as template using the primers 5-ACC CTT TTC AGA CAG AGC GA-3 and 5-CTT GAT TTC GTG AGC ACC AC-3. The obtained construct was designated pCZ25. The proper activity of COT1::MYC was verified by the ability of pCZ22 but not pCZ25 to complement the growth defects of cot-1(ts). Western-blot analysis using anti-MYC antibodies verified the presence of MYC-tagged COT1 with the expected molecular mass. 
Table IV-2 Neurospora crassa strains used in this study

\begin{tabular}{|c|c|c|}
\hline Strain & Genotype & Source \\
\hline wild type mat $\mathrm{A}$ & 74-OR23-1A & FGSC \#987 \\
\hline wild type mat a & ORS-SL6a & FGSC \#4200 \\
\hline $\cot -1(t s)$ & $\cot -1(H 351 R)$ & FGSC \#4066 \\
\hline myc-cot-1(EC) & $\cot -1(H 351 R) ;$ myc-cot-1::hph(EC) & Seiler et al., 2006 \\
\hline$m y c-\cot -1(t s)(E C)$ & cot-1(H351R);myc-cot-1(ts)::hph(EC) & this study \\
\hline pod-6(ts) & pod-6(I310K) & Seiler et al., 2006 \\
\hline$\Delta p o d-6$ & hph::pod-6s & Seiler et al., 2006 \\
\hline myc-cot-1 & myc::cot-1 & Maerz et al., 2009 \\
\hline$m y c-\cot -1(S 417 A)$ & $m y c:: \cot -1(S 417 A)$ & Ziv et al., 2009 \\
\hline myc-cot-1(S417E) & myc::cot-1(S4127E) & Ziv et al., 2009 \\
\hline myc-cot-1(T589A) & myc::cot-1(T589A) & Ziv et al., 2009 \\
\hline myc-cot-1(T589E) & myc::cot-1(T589E) & Ziv et al., 2009 \\
\hline myc-cot-1(D337A) & $m y c:: \cot -1(D 337 A)$ & Maerz et al., 2009 \\
\hline$\Delta m o b-2 a ; \Delta m o b-2 b$ & $h p h:: m o b-2 a \Delta ; h p h:: m o b-2 b \Delta$ & Maerz et al., 2009 \\
\hline$\Delta p o d-6 ; m y c-c o t-1(S 417 A)$ & myc::cot-1(S417A);hph::pod-64 & this study \\
\hline$\Delta p o d-6 ; m y c-c o t-1(S 417 E)$ & myc::cot-1(S417E);hph::pod-6A & this study \\
\hline spod-6;myc-cot-1(T589A) & myc::cot-1(T589A);hph::pod-6s & this study \\
\hline spod-6;myc-cot-1(T589E) & myc::cot-1(T589E);hph::pod-6s & this study \\
\hline \multirow[t]{2}{*}{$\Delta m o b-2 a ; \Delta m o b-2 b ; m y c-c o t-1(S 417 A)$} & $m y c:: \cot -1(S 417 A) ;$ & this study \\
\hline & $h p h:: m o b-2 a \Delta ; h p h:: m o b-2 b \Delta$ & \\
\hline \multirow[t]{2}{*}{$\Delta m o b-2 a ; \Delta m o b-2 b ; m y c-c o t-1(S 417 E)$} & $m y c:: \cot -1(S 417 E) ;$ & this study \\
\hline & $h p h:: m o b-2 a \Delta ; h p h:: m o b-2 b \Delta$ & \\
\hline \multirow[t]{2}{*}{$\Delta m o b-2 a ; \Delta m o b-2 b ; m y c-c o t-1(T 589 A)$} & myc::cot-1(T589A); & this study \\
\hline & $h p h:: m o b-2 a \Delta ; h p h:: m o b-2 b \Delta$ & \\
\hline \multirow[t]{2}{*}{$\Delta m o b-2 a ; \Delta m o b-2 b ; m y c-c o t-1(T 589 E)$} & myc::cot-1(T589E); & this study \\
\hline & $h p h:: m o b-2 a \Delta ; h p h:: m o b-2 b \Delta$ & \\
\hline
\end{tabular}

\section{Biochemical methods}

Purification of myc-tagged COT1 and peptide-based in vitro activity assays were performed as described previously (Maerz et al., 2009) with the following modifications. To test for COT1 kinase activity towards the activation segment (RSRRLMAYSTVGTPDYI) and the hydrophobic motif (EESPELSLPFIGYTFKRFDNNFR), the respective peptides were 
used at a final concentration of $2 \mathrm{mM}$ as substrates. The influence of modified hydrophobic motif based peptides on COT1 activity was analyzed by adding $1 \mathrm{mM}$ of HMA (EESPELSLPFIGYAFKRFDNNFR) or HM-D (EESPELSLPFIGYDFKRFDNNFR) to the respective kinase reaction mixture. An adjustment to a final concentration of $2 \%$ DMSO was necessary to prevent the formation of precipitates.

\section{Acknowledgment}

This research project was financially supported through the DFG Research Center of Molecular Physiology of the Brain (SS) and a joint DFG research grant SE1054/3-1 (SS and OY). We thank E. L. Weiss, Northwestern University, Evanston, Illinois, for the gift of the phospho-Ser417-COT1 specific anti-phospho-Ser570-Cbk1p antibody. 


\section{Supplementary Data}

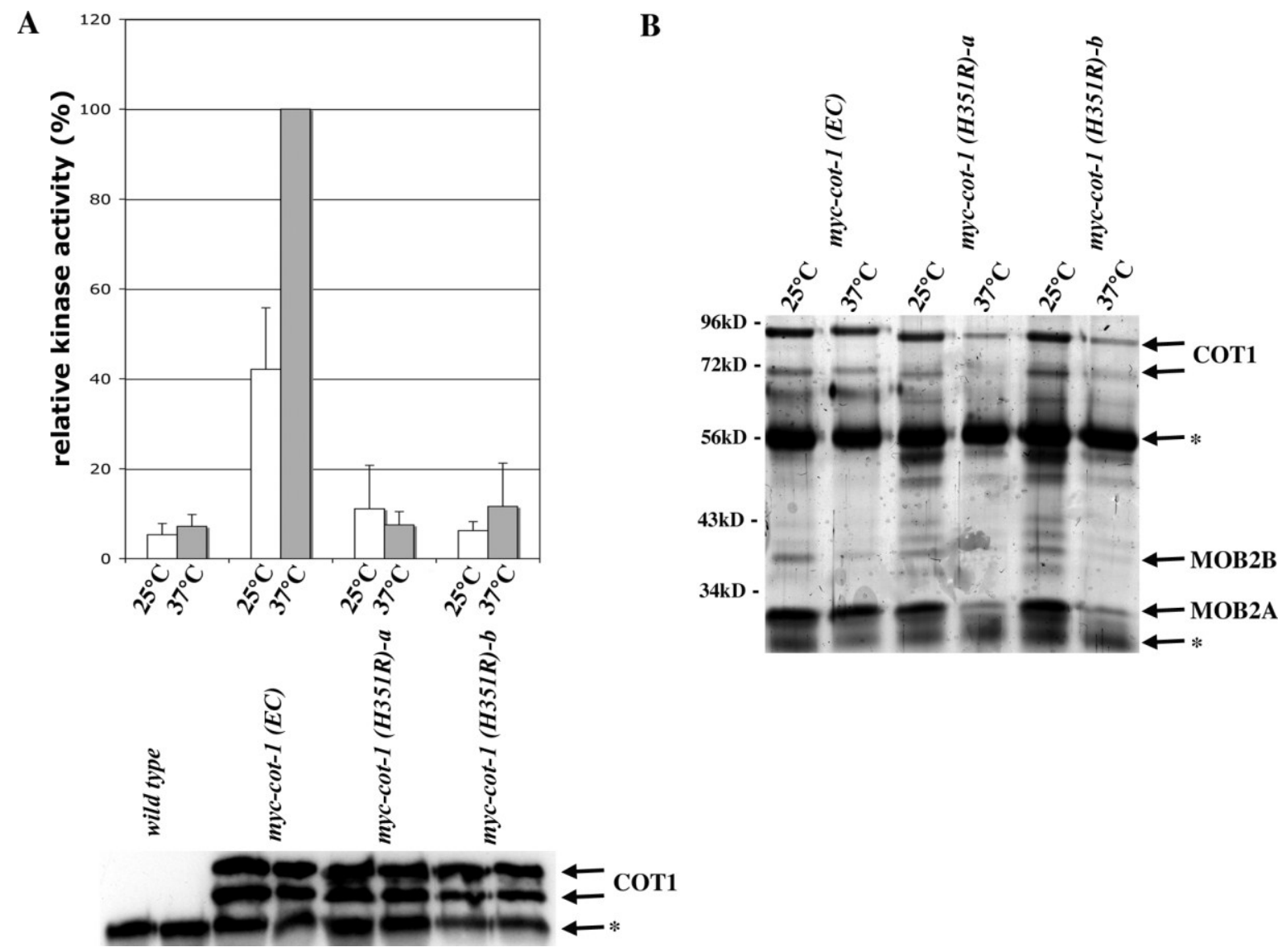

Figure S IV-1 Conditional COT1(H351R) lacks in vitro kinase activity, but its interaction with MOBs is not affected. (A) Kinase activity of immunoprecipitated myc-cot-1(H351R) from two independent strains (designated a and b) and myc-cot-1 cultured at $25^{\circ} \mathrm{C}$ and assayed at $25^{\circ} \mathrm{C}$ or $37^{\circ} \mathrm{C}$. Data are means of three independent experiments. (B) Copurification of associated MOB2 proteins in the myc-COT1 precipitant from the indicated strains. 


\section{References}

Behn-Krappa, A. \& Newton, A. C., (1999) The hydrophobic phosphorylation motif of conventional protein kinase $C$ is regulated by autophosphorylation. Curr Biol 9: 728-737.

Bichsel, S. J., Tamaskovic, R., Stegert, M. R. \& Hemmings, B. A., (2004) Mechanism of activation of NDR (nuclear Dbf2-related) protein kinase by the hMOB1 protein. $J$ Biol Chem 279: 35228-35235.

Biondi, R. M., Cheung, P. C., Casamayor, A., Deak, M., Currie, R. A. \& Alessi, D. R., (2000) Identification of a pocket in the PDK1 kinase domain that interacts with PIF and the C-terminal residues of PKA. EMBO J 19: 979-988.

Biondi, R. M. \& Nebreda, A. R., (2003) Signalling specificity of Ser/Thr protein kinases through docking-site-mediated interactions. Biochem J 372: 1-13.

Dan, I., Watanabe, N. M. \& Kusumi, A., (2001) The Ste20 group kinases as regulators of MAP kinase cascades. Trends Cell Biol 11: 220-230.

Davis, R., (2000) Neurospora: Contributions of a Model Organism. Oxford University Press, New York.

Davis, R. D. \& DeSerres, F. J., (1970) Genetic and microbiological research techniques for Neurospora crassa. Methods Enzymol. 17: 79-143.

Devroe, E., Erdjument-Bromage, H., Tempst, P. \& Silver, P. A., (2004) Human Mob proteins regulate the NDR1 and NDR2 serine-threonine kinases. J Biol Chem 279: 24444-24451.

Emoto, K., He, Y., Ye, B., Grueber, W. B., Adler, P. N., Jan, L. Y. \& Jan, Y. N., (2004) Control of dendritic branching and tiling by the Tricornered-kinase/Furry signaling pathway in Drosophila sensory neurons. Cell 119: 245-256.

Emoto, K., Parrish, J. Z., Jan, L. Y. \& Jan, Y. N., (2006) The tumour suppressor Hippo acts with the NDR kinases in dendritic tiling and maintenance. Nature 443: 210-213.

Engel, M., Hindie, V., Lopez-Garcia, L. A., Stroba, A., Schaeffer, F., Adrian, I., Imig, J., Idrissova, L., Nastainczyk, W., Zeuzem, S., Alzari, P. M., Hartmann, R. W., Piiper, A. \& Biondi, R. M., (2006) Allosteric activation of the protein kinase PDK1 with low molecular weight compounds. EMBO J 25: 5469-5480.

Frodin, M., Antal, T. L., Dummler, B. A., Jensen, C. J., Deak, M., Gammeltoft, S. \& Biondi, R. M., (2002) A phosphoserine/threonine-binding pocket in AGC kinases and PDK1 mediates activation by hydrophobic motif phosphorylation. EMBO J 21: 53965407.

Gallegos, M. E. \& Bargmann, C. I., (2004) Mechanosensory neurite termination and tiling depend on SAX-2 and the SAX-1 kinase. Neuron 44: 239-249.

Gao, T., Toker, A. \& Newton, A. C., (2001) The carboxyl terminus of protein kinase c provides a switch to regulate its interaction with the phosphoinositide-dependent kinase, PDK-1. J Biol Chem 276: 19588-19596. 
Geng, W., He, B., Wang, M. \& Adler, P. N., (2000) The tricornered gene, which is required for the integrity of epidermal cell extensions, encodes the Drosophila nuclear DBF2-related kinase. Genetics 156: 1817-1828.

Gorovits, R., Propheta, O., Kolot, M., Dombradi, V. \& Yarden, O., (1999) A mutation within the catalytic domain of COT1 kinase confers changes in the presence of two COT1 isoforms and in Ser/Thr protein kinase and phosphatase activities in Neurospora crassa. Fungal Genet Biol 27: 264-274.

Hanks, S. K. \& Hunter, T., (1995) Protein kinases 6. The eukaryotic protein kinase superfamily: kinase (catalytic) domain structure and classification. FASEB J 9: 576596.

He, Y., Emoto, K., Fang, X., Ren, N., Tian, X., Jan, Y. N. \& Adler, P. N., (2005a) Drosophila Mob family proteins interact with the related tricornered (Trc) and warts (Wts) kinases. Mol Biol Cell 16: 4139-4152.

He, Y., Fang, X., Emoto, K., Jan, Y. N. \& Adler, P. N., (2005b) The tricornered Ser/Thr protein kinase is regulated by phosphorylation and interacts with furry during Drosophila wing hair development. Mol Biol Cell 16: 689-700.

Hergovich, A., Bichsel, S. J. \& Hemmings, B. A., (2005) Human NDR kinases are rapidly activated by $\mathrm{MOB}$ proteins through recruitment to the plasma membrane and phosphorylation. Mol Cell Biol 25: 8259-8272.

Hergovich, A., Schmitz, D. \& Hemmings, B. A., (2006a) The human tumour suppressor LATS1 is activated by human MOB1 at the membrane. Biochem Biophys Res Commun 345: 50-58.

Hergovich, A., Stegert, M. R., Schmitz, D. \& Hemmings, B. A., (2006b) NDR kinases regulate essential cell processes from yeast to humans. Nat Rev Mol Cell Biol 7: 253-264.

Hirabayashi, S., Nakagawa, K., Sumita, K., Hidaka, S., Kawai, T., Ikeda, M., Kawata, A., Ohno, K. \& Hata, Y., (2008) Threonine 74 of MOB1 is a putative key phosphorylation site by MST2 to form the scaffold to activate nuclear Dbf2-related kinase 1. Oncogene 27: 4281-4292.

Hou, M. C., Guertin, D. A. \& McCollum, D., (2004) Initiation of cytokinesis is controlled through multiple modes of regulation of the Sid2p-Mob1p kinase complex. Mol Cell Biol 24: 3262-3276.

Jansen, J. M., Barry, M. F., Yoo, C. K. \& Weiss, E. L., (2006) Phosphoregulation of Cbk1 is critical for RAM network control of transcription and morphogenesis. J Cell Biol 175: 755-766.

Johnson, L. N., Noble, M. E. \& Owen, D. J., (1996) Active and inactive protein kinases: structural basis for regulation. Cell 85: 149-158.

Kanai, M., Kume, K., Miyahara, K., Sakai, K., Nakamura, K., Leonhard, K., Wiley, D. J., Verde, F., Toda, T. \& Hirata, D., (2005) Fission yeast MO25 protein is localized at SPB and septum and is essential for cell morphogenesis. EMBO J 24: 3012-3025.

Keranen, L. M., Dutil, E. M. \& Newton, A. C., (1995) Protein kinase C is regulated in vivo by three functionally distinct phosphorylations. Curr Biol 5: 1394-1403. 
Keshwani, M. M. \& Harris, T. K., (2008) Kinetic mechanism of fully activated S6K1 protein kinase. J Biol Chem 283: 11972-11980.

Lai, Z. C., Wei, X., Shimizu, T., Ramos, E., Rohrbaugh, M., Nikolaidis, N., Ho, L. L. \& Li, Y., (2005) Control of cell proliferation and apoptosis by mob as tumor suppressor, mats. Cell 120: 675-685.

Maerz, S., Dettmann, A., Ziv, C., Liu, Y., Valerius, O., Yarden, O. \& Seiler, S., (2009) Two NDR kinase - MOB complexes function as distinct modules during septum formation and tip extension in Neurospora crassa. Revised manuscript submitted; Mol. Microbiol.

McCluskey, K., (2003) The Fungal Genetics Stock Center: from molds to molecules. Adv Appl Microbiol 52: 245-262.

Nelson, B., Kurischko, C., Horecka, J., Mody, M., Nair, P., Pratt, L., Zougman, A., McBroom, L. D., Hughes, T. R., Boone, C. \& Luca, F. C., (2003) RAM: a conserved signaling network that regulates Ace2p transcriptional activity and polarized morphogenesis. Mol Biol Cell 14: 3782-3803.

Newton, A. C., (2003) Regulation of the ABC kinases by phosphorylation: protein kinase C as a paradigm. Biochem J 370: 361-371.

Oliver, A. W., Knapp, S. \& Pearl, L. H., (2007) Activation segment exchange: a common mechanism of kinase autophosphorylation? Trends Biochem Sci 32: 351-356.

Pike, A. C., Rellos, P., Niesen, F. H., Turnbull, A., Oliver, A. W., Parker, S. A., Turk, B. E., Pearl, L. H. \& Knapp, S., (2008) Activation segment dimerization: a mechanism for kinase autophosphorylation of non-consensus sites. EMBO J 27: 704-714.

Pombo, C. M., Force, T., Kyriakis, J., Nogueira, E., Fidalgo, M. \& Zalvide, J., (2007) The GCK II and III subfamilies of the STE20 group kinases. Front Biosci 12: 850-859.

Praskova, M., Xia, F. \& Avruch, J., (2008) MOBKL1A/MOBKL1B phosphorylation by MST1 and MST2 inhibits cell proliferation. Curr Biol 18: 311-321.

Saucedo, L. J. \& Edgar, B. A., (2007) Filling out the Hippo pathway. Nat Rev Mol Cell Biol 8: 613-621.

Seiler, S., Vogt, N., Ziv, C., Gorovits, R. \& Yarden, O., (2006) The STE20/germinal center kinase POD6 interacts with the NDR kinase COT1 and is involved in polar tip extension in Neurospora crassa. Mol Biol Cell 17: 4080-4092.

Shah, O. J. \& Hunter, T., (2004) Critical role of T-loop and H-motif phosphorylation in the regulation of S6 kinase 1 by the tuberous sclerosis complex. J Biol Chem 279: 20816-20823.

Stegert, M. R., Hergovich, A., Tamaskovic, R., Bichsel, S. J. \& Hemmings, B. A., (2005) Regulation of NDR protein kinase by hydrophobic motif phosphorylation mediated by the mammalian Ste20-like kinase MST3. Mol Cell Biol 25: 11019-11029.

Stegert, M. R., Tamaskovic, R., Bichsel, S. J., Hergovich, A. \& Hemmings, B. A., (2004) Regulation of NDR2 protein kinase by multi-site phosphorylation and the S100B calcium-binding protein. J Biol Chem 279: 23806-23812.

Toker, A. \& Newton, A. C., (2000) Akt/protein kinase B is regulated by autophosphorylation at the hypothetical PDK-2 site. J Biol Chem 275: 8271-8274. 
Vichalkovski, A., Gresko, E., Cornils, H., Hergovich, A., Schmitz, D. \& Hemmings, B. A., (2008) NDR kinase is activated by RASSF1A/MST1 in response to Fas receptor stimulation and promotes apoptosis. Curr Biol 18: 1889-1895.

Wei, X., Shimizu, T. \& Lai, Z. C., (2007) Mob as tumor suppressor is activated by Hippo kinase for growth inhibition in Drosophila. EMBO J 26: 1772-1781.

Yang, J., Cron, P., Good, V. M., Thompson, V., Hemmings, B. A. \& Barford, D., (2002a) Crystal structure of an activated Akt/protein kinase B ternary complex with GSK3peptide and AMP-PNP. Nat Struct Bio/ 9: 940-944.

Yang, J., Cron, P., Thompson, V., Good, V. M., Hess, D., Hemmings, B. A. \& Barford, D., (2002b) Molecular mechanism for the regulation of protein kinase B/Akt by hydrophobic motif phosphorylation. Mol Cell 9: 1227-1240.

Yarden, O., Plamann, M., Ebbole, D. J. \& Yanofsky, C., (1992) cot-1, a gene required for hyphal elongation in Neurospora crassa, encodes a protein kinase. EMBO J 11: 2159-2166.

Zallen, J. A., Peckol, E. L., Tobin, D. M. \& Bargmann, C. I., (2000) Neuronal cell shape and neurite initiation are regulated by the $\mathrm{Ndr}$ kinase SAX-1, a member of the Orb6/COT-1/warts serine/threonine kinase family. Mol Biol Cell 11: 3177-3190.

Ziv, C., Kra-Oz, G., Gorovits, R., Maerz, S., Seiler, S. \& Yarden, O., (2009) Cell elongation and branching are regulated by differential phosphorylation states of the NDR kinase COT1 in Neurospora crassa. Revised manuscript submitted; Mol. Microbiol. 


\section{DANKSAGUNG}

Bedanken möchte ich mich bei all jenen, die mich bei meiner Arbeit unterstützt und mir stets mit Rat und Tat zur Seite standen, zuerst bei meinem Doktorvater Prof. Gerhard Braus, in dessen Abteilung ich meine Dissertation angefertigt habe, sowie Prof. Stefanie Pöggeler, für die Übernahme des Korreferats.

Ebenso möchte ich mich auch bei allen Angehörigen der Abteilung für ihre Hilfe, aber auch interessante Gespräche bedanken, insbesondere bei Nicole Scheiter für ihre super Arbeit im Bestellwesen, ohne sie wäre vieles ungleich komplizierter gewesen.

Auch meine Familie hat ihren Beitrag an dieser Arbeit geleistet, sei es durch Rückhalt, Ablenkung oder aber diverse Diskussionen. Mein Dank ist daher auch an meine Eltern und meine Geschwister gerichtet.

In besonderem Maße möchte ich mich aber bei allen Mitgliedern, auch den ehemaligen, bedanken. Sie alle tragen einen sehr großen Anteil am Gelingen meiner Arbeit. Stephan, mein direkter Betreuer, hat sich immer Zeit für die größeren und kleineren Probleme des Laboralltages genommen. Danni, Anne, Corinna, Immo, Nico und all die anderen die mich auf meinen Weg begleiteten, sorgten durch eine gute Atmosphäre, konstruktive und abwechslungsreiche Gespräche, sogar durch kleinere Reibereien dafür, dass ich die Zeit meiner Doktorarbeit für immer positiv in Erinnerung haben werde.

\section{Vielen Dank.}

Das Leben des Forschers ist manchmal voller Verdruss, weil man auch Fehlschläge hinnehmen muss, Doch mit besonderen Freunden und des Betreuers gutem Rat schritt motiviert ich immer voran zu neuer Tat.

Auch Erfolg und viel Freude bereitet das Forscherleben Wissen zu erhalten und Wissen zu vergeben.

Dank an Danni, die durch dick und dünn mich hat begleitet, und mir dadurch viel Freude hat bereitet.

Dank auch an Anne, unseren Laborsonnenschein, wie wird es wohl später ohne euch beide sein?

Dank auch an Stephan, der fast immer sprang, wenn ich mal rief, aber dadurch wahrscheinlich auch vieles gut lief. Dank an die anderen, auch ehemaligen Labormitglieder, zusammenarbeiten würde ich mit euch immer wieder. 


\section{Curriculum Vitae}

\section{Sabine März}

$\begin{array}{ll}\text { Geburtsdatum: } & 05.06 .1980 \\ \text { Geburtsort: } & \text { Weimar } \\ \text { Staatsangehörigkeit: } & \text { deutsch }\end{array}$

Doktorarbeit am Institut für Mikrobiologie und Genetik der Georg-August-Universität Göttingen

in der Abteilung für molekulare Mikrobiologie und Genetik (Prof. G. Braus)

Thema: Regulation of fungal polar tip extension through NDR kinase signalling

Oktober 2004 - Mai 2005

Oktober 1999 - Oktober 2004

August 1991 - Juli 1999

September 1987 - August 1991
Anstellung als wissenschaftlicher Mitarbeiter an der Friedrich-Schiller-Universität Jena

Biochemiestudium an der Friedrich-SchillerUniversität Jena

Abschluss: Diplom-Biochemiker

Wahlpflichtfach: Molekulare Medizin

Thema der Diplomarbeit: Interaktion der Proteinkinase C mit dem Signaling-Modul von EGF-Rezeptor, Src und PLC $\gamma-1$

Prof.-Fritz-Hofmann-Gymnasium, Kölleda

Grundschule, Buttstädt 